\title{
The Evolution Problem in General Relativity
}

\author{
Mihalis Dafermos*
}

ABstract. These notes accompany a set of lectures for the Current

Developments in Mathematics conference, Harvard, November 22, 2008.

\section{Contents}

1. Introduction: General relativity and evolution 3

1.1. The Einstein equations 3

1.2. The Cauchy problem 4

1.3. Explicit solutions: Minkowski, Schwarzschild, Kerr 4

1.4. Dynamics and the stability problem 5

1.5. Outline 6

2. The Cauchy problem in general relativity 6

2.1. The constraint equations 6

2.2. Initial data $\quad 7$

2.2.1. The vacuum case $\quad 7$

2.2.2. The case of matter 7

2.2.3. Asymptotic flatness and the positive mass theorem 7

2.3. The maximal development 9

2.4. Harmonic coordinates and the proof of local existence 10

2.5. Stability of Minkowski space 11

2.6. Penrose's incompleteness theorem 12

3. Schwarzschild and Kerr 13

3.1. The Schwarzschild metric 13

3.1.1. Schwarzschild's stars 13

3.1.2. The maximal extension of Synge and Kruskal 14

3.1.3. The Penrose diagram of Schwarzschild 16

3.1.4. The black hole concept 17

3.1.5. Negative mass Schwarzschild and weak cosmic censorship 20

*University of Cambridge, Department of Pure Mathematics and Mathematical Statistics, Wilberforce Road, Cambridge CB3 0WB United Kingdom.

(C)2009 International Press 
3.2. The Kerr metric $\quad 22$

3.2.1. Boyer-Lindquist coordinates 22

3.2.2. The ergoregion 24

3.2.3. The Cauchy horizon and strong cosmic censorship 24

4. The black hole stability problem 26

4.1. The nonlinear stability of Kerr conjecture 26

4.2. Introduction to $\square_{g} \psi=0 \quad 27$

4.2.1. The Kay-Wald boundedness theorem 29

4.3. The red-shift and a new proof of boundedness 31

4.3.1. The classical red-shift 32

4.3.2. The redshift effect as a multiplier and commutator estimate 32

4.3.3. A robust boundedness statement 33

4.3.4. Perturbing? 34

4.4. Quantitative decay for the wave equation on Schwarzschild 34

4.4.1. The $X$-multiplier and integral decay 35

4.4.2. The $Z$-multiplier $\quad 37$

4.4.3. Pointwise estimates $\quad 38$

4.4.4. Comments and further reading $\quad 39$

4.4.5. Perturbing? 39

4.4.6. Aside: Quantitative vs. non-quantitative results and the heuristic tradition $\quad 39$

4.5. Boundedness for axisymmetric black holes 41

4.5.1. A geometric property of the horizon 42

4.5.2. The boundedness theorem 42

4.5.3. The axisymmetric case 43

4.5.4. Superradiant and non-superradiant frequencies 43

4.5.5. Cutoff and decomposition $\quad 46$

4.5.6. Pointwise bounds 46

4.6. Quantitative decay for waves on Kerr 46

4.7. Comments and further reading 49

4.8. The cosmological constant $\Lambda$ and Schwarzschild-de Sitter 50

4.8.1. The Schwarzschild-de Sitter geometry 51

4.8.2. Boundedness and decay 51

4.8.3. Comments and further reading $\quad 52$

5. Open problems $\quad 53$

5.1. The wave equation 53

5.2. Higher spin 54

5.3. The Klein-Gordon equation $\quad 55$

5.4. Asymptotically anti-de Sitter spacetimes 55

5.5. Higher dimensions 56

$\begin{array}{ll}\text { 5.6. Nonlinear problems } & 57\end{array}$

6. Acknowledgements $\quad 58$

$\begin{array}{ll}\text { Appendix A. Lorentzian geometry } & 58\end{array}$

A.1. The Lorentzian signature 58 
A.2. Time-orientation and causality 59

A.3. Covariant derivatives, geodesics, curvature $\quad 59$ References

\section{Introduction: General relativity and evolution}

The formulation of general relativity represents a revolutionary development in the foundations of physics, unifying geometry, inertia, and gravitation as aspects of a single structure: a Lorentzian metric $g_{\mu \nu}$ on a 4-dimensional continuum $\mathcal{M}$ known as spacetime. Nonetheless, general relativity can be viewed as quintessentially classical, the culmination of the field-theoretic world-view where physics is governed by a closed system of partial differential equations with a well-posed initial value problem. The remarkable geometric structure of the theory notwithstanding, the fundamental problem in general relativity thus remains unchanged from Newtonian mechanics of point particles: Understand the future from initial conditions. This is the problem of dynamics.

1.1. The Einstein equations. We review briefly the structure of the theory. General relativity postulates a 4-dimensional Lorentzian manifold $(\mathcal{M}, g)$-space-time-which is to satisfy the Einstein equations

$$
R_{\mu \nu}-\frac{1}{2} g_{\mu \nu} R=8 \pi T_{\mu \nu} .
$$

Here, $R_{\mu \nu}, R$ denote the Ricci and scalar curvature of $g$, respectively, and $T_{\mu \nu}$ denotes a symmetric 2 -tensor on $\mathcal{M}$ termed the stress-energy-momentum tensor of matter. (Necessary background on Lorentzian geometry to understand the above notation is given in Appendix A.) The equations (1) in of themselves do not close, but must be coupled to "matter equations" satisfied by a collection $\left\{\Psi_{i}\right\}$ of matter fields defined on $\mathcal{M}$, together with a constitutive relation determining $T_{\mu \nu}$ from $\left\{g, \Psi_{i}\right\}$. These equations and relations are stipulated by the relevant continuum field theory (electromagnetism, fluid dynamics, etc.) describing the matter.

Einstein was led to the system (1) in 1915, after a 7-year struggle to incorporate gravity into his earlier principle of relativity. In the field-theoretic formulation of the "Newtonian" theory, gravity was described by the Newtonian potential $\phi$ satisfying the Poisson equation

$$
\triangle \phi=4 \pi \mu,
$$

where $\mu$ denotes the mass-density of matter. It is truly remarkable that the constraints of consistency were so rigid that encorporating gravitation required finally a complete reworking of the principle of relativity, leading to a theory where Newtonian gravity, special relativity and Euclidean geometry each emerge as limiting aspects of one dynamic geometrical structure-the Lorentzian metric-naturally living on a 4-dimensional spacetime continuum. 
A second remarkable aspect of general relativity is that, in contrast to its Newtonian predecessor, the theory is non-trivial even in the absence of matter. In that case, we set $T_{\mu \nu}=0$ and the system (1) takes the form

$$
R_{\mu \nu}=0 .
$$

The equations (3) are known as the Einstein vacuum equations. Whereas (2) is a linear elliptic equation, (3) can be seen to form a closed system of nonlinear (but quasilinear) wave equations. Essentially all of the characteristic features of the dynamics of the Einstein equations are already present in the study of the vacuum equations (3). This fact is very appealing for the mathematician who does not wish to dirty his hands with matter! Equations (3) express a relation of pure geometry. See also the discussion in [55].

1.2. The Cauchy problem. The natural problem for (3) is the Cauchy problem. In contrast, however, to other non-linear field theories arising in physics, in the case of general relativity, even formulating the Cauchy problem requires addressing several conceptual issues (e.g. what is the appropriate notion of initial data, in what sense is (3) hyperbolic?), and these took a long time to be correctly sorted out. Important advances in this process include the identification of the harmonic gauge by de Donder [65], the existence and uniqueness theorems for general quasilinear wave equations in the 1930's based on work of Friedrichs, Schauder, Sobolev, Petrovsky, Leray and others, and Leray's notion of global hyperbolicity [103]. The well-posedness of the appropriate Cauchy problem for the vacuum equations (3) was finally formulated and proven in celebrated work of Choquet-Bruhat [30] (1952) and Choquet-Bruhat-Geroch [32] (1969). We shall give in Section 2 a concise survey of these developments, the precise statement of the existence and uniqueness theorems and some comments on their proof.

1.3. Explicit solutions: Minkowski, Schwarzschild, Kerr. A much more elementary approach to understanding a nonlinear theory like (3) than studying the general Cauchy problem is to identify important explicit solutions, i.e., solutions which can be written in closed form. ${ }^{1}$ Not surprisingly, much of the early history of general relativity centred around the discovery and interpretation of such solutions. The simplest explicit solution to the Einstein vacuum equations (3) is Minkowski space, typically denoted by $\mathbb{R}^{3+1}$. This solution predates the formulation of general relativity. It was in fact precisely Minkowski's discovery [108] that Einstein's earlier "special" relativity principle could be described by the metric

$$
-d t^{2}+d x^{2}+d y^{2}+d z^{2}
$$

on the four-dimensional $\mathbb{R}^{4}$ which introduced the spacetime concept and paved the way for the formulation of general relativity.

The next simplest solution of (3) is the so-called Schwarzschild solution, written down [131] already in 1916. This is in fact a one-parameter family of

\footnotetext{
${ }^{1}$ The traditional terminology in general relativity for such solutions is exact solutions.
} 
solutions $\left(\mathcal{M}, g_{M}\right)$, the parameter $M$ identified with mass. See (15) below for the metric form. The Schwarzschild family lives as a subfamily in a larger two-parameter family of explicit solutions $\left(\mathcal{M}, g_{M, a}\right)$ known as the Kerr solutions, discussed in Section 3.2. These were discovered only much later [90] (1963).

When the Schwarzschild solution was first written down in local coordinates, the necessary concepts to understand its geometry had not yet been developed. It took nearly 50 years from the time when Schwarzschild was first discovered for its global geometry to be sufficiently well understood so as to be given a suitable name: Schwarzschild and Kerr were examples of what came to be known as black hole spacetimes. ${ }^{2}$ The Schwarzschild and Kerr solutions also illustrate another feature of solutions to the Einstein equations, namely, they exhibit geodesic incompleteness. This incompleteness turns out to be a very general feature, as was shown by Penrose in a celebrated theorem (Theorem 2.6 below). In Schwarzschild, the curvature blows up along all incomplete geodesics. In Kerr, the origin of incompleteness is purely global. The question of interpreting Penrose's incompleteness theorem naturally gives rise to the celebrated weak and strong cosmic censorship conjectures. We will formulate these in Sections 3.1.5 and 3.2.3.

1.4. Dynamics and the stability problem. Explicit solutions are suggestive of general features of dynamics, but only if they are stable. This notion can in turn only be formulated in the context of the Cauchy problem discussed above: A family of solutions $\mathcal{F}$ is stable if the solution arising from any Cauchy data set sufficiently close to the Cauchy data of a member of $\mathcal{F}$ tends asymptotically to another member of $\mathcal{F}$ in a suitable sense.

The stability of Minkowski space was first proven in the monumental work of Christodoulou and Klainerman [46]. See Section 2.5 for a formulation of this result. The dynamical stability of the Kerr family ${ }^{3}$ as a family of solutions to the Cauchy problem for the Einstein equations, even restricted to parameter values near Schwarzschild, i.e. $|a| \ll M$, is yet to be understood and poses an important challenge for the mathematical study of general relativity in the coming years. See Section 4.1 for a formulation of this problem. In fact, even the most basic linear properties of waves, for instance, general solutions of

$$
\square_{g} \psi=0
$$

on Schwarzschild or Kerr spacetime backgrounds (or more generally, backgrounds near Kerr) have only recently been understood. In view of the wavelike features of the Einstein equations (3) (see in particular Section 2.4), this latter problem should be thought of as a prerequisite for understanding the non-linear stability problem.

\footnotetext{
${ }^{2}$ This name is due to John Wheeler.

${ }^{3}$ Note that (without restricting to symmetry) one cannot study the stability problem for Schwarzschild per se. Only the larger Kerr family can be stable.
} 
1.5. Outline. This article will give an overview of aspects of the evolution problem in general relativity. Section 2 will review general results on the Cauchy problem, including a discussion of the stability of Minkowski space and Penrose's incompleteness theorem. Section 3 will discuss the black hole concept via the examples of Schwarzschild and Kerr. This discussion will lead naturally to the formulations of the weak and strong cosmic censorship conjectures. Section 4 will begin with a formulation of the nonlinear stability of Kerr conjecture, and then pass to the study of the linear problem (4) on Schwarzschild, Kerr, and more general spacetime backgrounds near Kerr. This is the most basic linear problem that must be understood well if one is to approach the nonlinear stability conjecture. The theorems of Sections 4.3-4.8 are due to the author in collaboration with Igor Rodnianski. Finally, Section 5 provides a list of open problems. It is hoped that these lectures contribute to the point of view that puts general relativity at the centre of modern developments in partial differential equations of evolution.

\section{The Cauchy problem in general relativity}

This section will outline the basic framework of the Cauchy problem for the Einstein equations

$$
R_{\mu \nu}-\frac{1}{2} g_{\mu \nu} R+\Lambda g_{\mu \nu}=8 \pi T_{\mu \nu}
$$

Here $\Lambda$ is a constant known as the cosmological constant and $T_{\mu \nu}$ is the so-called energy momentum tensor of matter. We shall consider mainly the vacuum case

$$
R_{\mu \nu}=\Lambda g_{\mu \nu}
$$

where the system closes in itself. In the original formulation of the theory, $\Lambda=0$. See the discusssion of Section 4.8. The case of matter will be illustrated by the example of a scalar field.

2.1. The constraint equations. Let $\Sigma$ be a spacelike hypersurface in $(\mathcal{M}, g)$, with future directed unit timelike normal $N$. By definition, $\Sigma$ inherits a Riemannian metric from $g$. On the other hand, the so-called second fundamental form of $\Sigma$ is defined to be the symmetric covariant 2tensor in $T \Sigma$ given by

$$
K(u, v)=-g\left(\nabla_{u} V, N\right)
$$

where $V$ denotes an arbitrary extension of $v$ to a vector field along $\Sigma$, and $\nabla$ here denotes the connection of $g$. As in Riemannian geometry, one easily shows that the above indeed defines a tensor on $T \Sigma$, and that it is symmetric.

Suppose now $(\mathcal{M}, g)$ satisfies $(5)$ with some tensor $T_{\mu \nu}$. With $\Sigma$ as above, let $\bar{g}_{a b}, \bar{\nabla}, K_{a b}$ denote the induced metric, connection, and second fundamental form, respectively, of $\Sigma$. Let barred quantities and Latin indices 
refer to tensors, curvature, etc., on $\Sigma$, and let $\Pi_{a}^{\nu}(p)$ denote the components of the pullback map $T^{*} \mathcal{M} \rightarrow T^{*} \Sigma$. It follows that

$$
\begin{aligned}
\bar{R}+\left(K_{a}^{a}\right)^{2}-K_{b}^{a} K_{a}^{b} & =16 \pi T_{\mu \nu} n^{\mu} n^{\nu}+2 \Lambda, \\
\nabla_{b} K_{a}^{b}-\nabla_{a} K_{b}^{b} & =16 \pi \Pi_{a}^{\nu} T_{\mu \nu} n^{\mu} .
\end{aligned}
$$

To see this, one derives as in Riemannian geometry the Gauss and Codazzi equations, takes traces, and applies (5).

2.2. Initial data. It is clear that $(7),(8)$ are necessary conditions on the induced geometry of a spacelike hypersurface $\Sigma$ so as to arise as a hypersurface in a spacetime satisfying (5). As shall be seen immediately below, they will also be sufficient conditions for solving the initial value problem.

2.2.1. The vacuum case. Let $\Sigma$ be a 3 -manifold, $\bar{g}$ a Riemannian metric on $\Sigma$, and $K$ a symmetric covariant 2 -tensor. Let us call $(\Sigma, \bar{g}, K)$ a vacuum initial data set with cosmological constant $\Lambda$ if (7)-(8) are satisfied with $T_{\mu \nu}=0$. Note that in this case, equations (7)-(8) refer only to $\Sigma, \bar{g}, K$.

2.2.2. The case of matter. Let us here provide only the case for the Einstein-scalar field case. Here, the system is (5) coupled with

$$
\begin{aligned}
\square_{g} \psi & =0, \\
T_{\mu \nu} & =\partial_{\mu} \psi \partial_{\nu} \psi-\frac{1}{2} g_{\mu \nu} \nabla^{\alpha} \psi \nabla_{\alpha} \psi .
\end{aligned}
$$

First note that were $\Sigma$ a spacelike hypersurface in a spacetime $(\mathcal{M}, g)$ satisfying the Einstein-scalar field system with massless scalar field $\psi$, and $n^{\mu}$ were the future-directed normal, then setting $\psi^{\prime}=n^{\mu} \partial_{\mu} \phi, \psi=\left.\phi\right|_{\Sigma}$, it follows that

$$
\begin{aligned}
T_{\mu \nu} n^{\mu} n^{\nu} & =\frac{1}{2}\left(\left(\psi^{\prime}\right)^{2}+\bar{\nabla}^{a} \psi \bar{\nabla}_{a} \psi\right), \\
\Pi_{a}^{\nu} T_{\mu \nu} n^{\mu} & =\psi^{\prime} \bar{\nabla}_{a} \psi,
\end{aligned}
$$

where latin indices and barred quantities refer to $\Sigma$ and its induced metric and connection.

This motivates the following: Let $\Sigma$ be a 3-manifold, $\bar{g}$ a Riemannian metric on $\Sigma, K$ a symmetric covariant 2 -tensor, and $\psi: \Sigma \rightarrow \mathbb{R}, \psi^{\prime}$ : $\Sigma \rightarrow \mathbb{R}$ functions. The triple $(\Sigma, \bar{g}, K)$ is said to be an Einstein-scalar field initial data set with cosmological constant $\Lambda$ if (7)-(8) are satisfied replacing $T_{\mu \nu} n^{\mu} n^{\nu}$ with $\frac{1}{2}\left(\left(\psi^{\prime}\right)^{2}+\bar{\nabla}^{a} \psi \bar{\nabla}_{a} \psi\right)$, and replacing $\Pi_{a}^{\nu} T_{\mu \nu} n^{\mu}$ with $\psi^{\prime} \bar{\nabla}_{a} \psi$.

Note again that with the above replacements the equations (7)-(8) do not refer to an ambient spacetime $\mathcal{M}$.

2.2.3. Asymptotic flatness and the positive mass theorem. The study of the Einstein constraint equations is non-trivial!

Let us refer in this section to a triple $(\Sigma, \bar{g}, K)$ where $\Sigma$ is a 3-manifold, $\bar{g}$ a Riemannian metric, and $K$ a symmetric two-tensor on $\Sigma$ as an initial data set, even though we have not specified a particular closed system of equations. An initial data set $(\Sigma, \bar{g}, K)$ is strongly asymptotically flat with 
one end if there exists a compact set $\mathcal{K} \subset \Sigma$ and a coordinate chart on $\Sigma \backslash \mathcal{K}$ which is a diffeomorphism to the complement of a ball in $\mathbb{R}^{3}$, and for which

$$
g_{a b}=\left(1+\frac{2 M}{r}\right) \delta_{a b}+o_{2}\left(r^{-1}\right), \quad k_{a b}=o_{1}\left(r^{-2}\right)
$$

where $\delta_{a b}$ denotes the Euclidean metric and $r$ denotes the Euclidean polar coordinate.

The assumption of asymptotic flatness is an idealization for "isolated self-gravitating systems". For the case of cosmology, where spacetime is to represent the whole universe, see Section 4.8.

In appopriate units, $M$ is the "mass" measured by asymptotic observers, when comparing to Newtonian motion in the frame $\delta_{a b}$. On the other hand, under the assumption of a global coordinate system well-behaved at infinity, $M$ can be computed by integration of the $t_{0}^{0}$ component of a certain pseudotensor ${ }^{4}$ added to $T_{0}^{0}$. In this manifestation, the quantity $E=M$ is known as the total energy. ${ }^{5}$ This relation was first studied by Einstein and is discussed in Weyl's [144]. If one looks at $E$ for a family of hypersurfaces with the above asymptotics, then $E$ is conserved.

A celebrated theorem of Schoen-Yau $[\mathbf{1 2 9}, \mathbf{1 3 0}]$ (see also [146]) states

THEOREM 2.1. Let $(\Sigma, \bar{g}, K)$ be strongly asymptotically flat with one end and satisfy (7), (8) with $\Lambda=0$, and where $T_{\mu \nu} n^{\mu} n^{\nu}, \Pi_{a}^{\nu} T_{\mu \nu} n^{\mu}$ are replaced by the scalar $\mu$ and the tensor $J_{a}$, respectively, defined on $\Sigma$, such that moreover $\mu \geq \sqrt{J^{a} J_{a}}$. Suppose moreover the asymptotics are strengthened by replacing $o_{2}\left(r^{-1}\right)$ by $O_{4}\left(r^{-2}\right)$ and $o_{1}\left(r^{-2}\right)$ by $O_{3}\left(r^{-3}\right)$. Then $M \geq 0$ and $M=0$ iff $\Sigma$ embeds isometrically into $\mathbb{R}^{3+1}$ with induced metric $\bar{g}$ and second fundamental form $K$.

The assumption $\mu \geq \sqrt{J^{a} J_{a}}$ holds if the matter satisfies the dominant energy condition [81]. In particular, it holds for the Einstein scalar field system of Section 2.2.2, and (of course) for the vacuum case. The statement we have given above is weaker than the full strength of the Schoen-Yau result. For the most general assumptions under which mass can be defined, see $[\mathbf{9}]$.

One can define the notion of strongly asymptotically flat with $k$ ends by assuming that there exists a compact $\mathcal{K}$ such that $\Sigma \backslash \mathcal{K}$ is a disjoint union of $k$ regions possessing a chart as in the above definition. The Cauchy surface $\Sigma$ of Schwarzschild of Kerr with $0 \leq|a|<M$, can be chosen to be strongly

\footnotetext{
${ }^{4}$ This is subtle: The Einstein vacuum equations arise from the Hilbert Lagrangian $\mathcal{L}(g)=\int R$ which is 2 nd order in the metric. In local coordinates, the highest order term is a divergence, and the Lagrangian can thus be replaced by a new Lagrangian which is 1st order in the metric. The resulting Lagrangian density, however, is no longer coordinate invariant. The quantity $t_{0}^{0}$ now arises from "Noether's theorem" [116]. See [44] for a nice discussion.

${ }^{5}$ With the above asymptotics, the so-called linear momentum vanishes. Thus, in this case "mass" and energy are equivalent.
} 
asymptotically flat with 2-ends. The mass of both ends coincides with the parameter $M$ of the solution.

The above theorem applies to this case as well for the parameter $M$ associated to any end. If $M=0$ for one end, then it follows by the rigidity statement that there is only one end.

In the presence of black holes, one expects a strengthening of the lower bound on mass in Theorem 2.1 to include a term related to the square root of the area of a cross section of the horizon. Such inequalities were first discussed by Penrose [119] with the Bondi mass in place of the mass defined above. All inequalities of this type are often called Penrose inequalities. It is not clear what this term should be, as the horizon is only identifyable after global properties of the maximal development have been understood. Thus, one often replaces this area in the conjectured inequality with the area of a suitably defined apparent horizon. Such a statement has indeed been obtained in the so-called Riemannian case (corresponding to $K=0$ ) where the relevant notion of apparent horizon coincides with that of minimal surface. See the important papers of Huisken-Ilmanen [86] and Bray [22].

2.3. The maximal development. Let $(\Sigma, \bar{g}, K)$ denote a smooth vacuum initial data set with cosmological constant $\Lambda$. We say that a smooth spacetime $(\mathcal{M}, g)$ is a smooth development of initial data if

(1) $(\mathcal{M}, g)$ satisfies the Einstein vacuum equations (3) with cosmological constant $\Lambda$.

(2) There exists a smooth embedding $i: \Sigma \rightarrow \mathcal{M}$ such that $(\mathcal{M}, g)$ is globally hyperbolic with Cauchy surface $i(\Sigma)$, and $\bar{g}, K$ are the induced metric and second fundamental form, respectively.

The original local existence and uniqueness theorems were proven in 1952 by Choquet-Bruhat $[\mathbf{3 0}] .{ }^{6}$ In modern language, they can be formulated as follows

TheOREM 2.2. Let $(\Sigma, \bar{g}, K)$ be as in the statement of the above theorem. Then there exists a smooth development $(\mathcal{M}, g)$ of initial data.

Theorem 2.3. Let $\mathcal{M}, \widetilde{\mathcal{M}}$ be two smooth developments of initial data. Then there exists a third development $\mathcal{M}^{\prime}$ and isometric embeddings $j$ : $\mathcal{M}^{\prime} \rightarrow \mathcal{M}, \tilde{j}: \mathcal{M}^{\prime} \rightarrow \widetilde{\mathcal{M}}$ commuting with $i, \tilde{i}$.

Application of Zorn's lemma, the above two theorems and simple facts about Lorentzian causality yields:

Theorem 2.4. (Choquet-Bruhat-Geroch $[\mathbf{3 2}])$ Let $(\Sigma, \bar{g}, K)$ denote a smooth vacuum initial data set with cosmological constant $\Lambda$. Then there exists a unique development of initial data $(\mathcal{M}, g)$ satisfying the following maximality statement: If $(\widetilde{\mathcal{M}}, \widetilde{g})$ satisfies (1), (2) with embedding $\tilde{i}$, then there exists an isometric embedding $j: \widetilde{\mathcal{M}} \rightarrow \mathcal{M}$ such that $j$ commutes with $\tilde{i}$.

\footnotetext{
${ }^{6}$ Then called Fourès-Bruhat.
} 
The spacetime $(\mathcal{M}, g)$ is known as the maximal development of $(\Sigma, \bar{g}, K)$. The spacetime $\mathcal{M} \cap J^{+}(\Sigma)$ is known as the maximal future development and $\mathcal{M} \cap J^{-}(\Sigma)$ the maximal past development.

We have formulated the above theorems in the class of smooth initial data. They are of course proven in classes of finite regularity. There has been much recent work in proving a version of Theorem 2.2 under minimal regularity assumptions. The current state of the art requires only $\bar{g} \in H^{2+\epsilon}$, $K \in H^{1+\epsilon}$. See $[\mathbf{9 3}]$.

We leave to the reader the task of formulating the analogue of Theorem 2.4 for the Einstein-scalar field system (5), (9), (10), where the notion of initial data set is that given in Section 2.2.2.

2.4. Harmonic coordinates and the proof of local existence. The statements of Theorems 2.2 and 2.3 are coordinate independent. Their proofs, however, require fixing a gauge which determines the form of the metric functions in coordinates from initial data. The classic gauge is the so-called harmonic gauge. ${ }^{7}$ Here the coordinates $x^{\mu}$ are required to satisfy

$$
\square_{g} x^{\mu}=0 .
$$

Equivalently, this gauge is characterized by the condition

$$
g^{\mu \nu} \Gamma_{\mu \nu}^{\alpha}=0 .
$$

A linearised version of this coordinate condition was used by Einstein [69] to predict gravitational waves. It appears that de Donder [65] was the first to consider harmonic coordinates in general.

The result of Theorem 2.3 actually predates Theorem 2.2 , and in some form was first proven by Stellmacher [133]. Given two developments $(\mathcal{M}, g)$, $(\widetilde{\mathcal{M}}, \tilde{g})$ one constructs for each harmonic coordinates $x^{\mu}, \tilde{x}^{\mu}$ adapted to $\Sigma$, such that $g_{\mu \nu}=\tilde{g}_{\mu \nu}, \partial_{\lambda} g_{\mu \nu}=\partial_{\lambda} \tilde{g}_{\mu \nu}$ along $\Sigma$. In these coordinates, the Einstein vaccum equations can be expressed as

$$
\square_{g} g^{\mu \nu}=Q_{\iota \kappa \lambda \rho \sigma \tau}^{\mu \nu, \alpha \beta} g^{\iota \kappa} \partial_{\alpha} g^{\lambda \rho} \partial_{\beta} g^{\sigma \tau}
$$

for which uniqueness follows from general results of Schauder [128]. This theorem gives in addition a domain of dependence property. ${ }^{8}$

Existence for solutions of the system (13) with smooth initial data would also follow from the results of Schauder [128]. This does not immediately yield a proof of Theorem 2.2, because one does not have a priori the spacetime metric $g$ so as to impose (11) or (12)! The crucial observation is that if (12) is true "to first order" on $\Sigma$, and $g$ is defined to be the unique solution

\footnotetext{
${ }^{7}$ Also known as wave coordinates.

${ }^{8}$ There is even earlier work on uniqueness in the analytic category going back to Hilbert, appealing to Cauchy-Kovalevskaya. Unfortunately, nature is not analytic; in particular, one cannot infer the domain of dependence property from those considerations.
} 
to (13), then (12) will hold, and thus, $g$ will solve (5). Thus, to prove Theorem 2.2, it suffices to show that one can arrange for (12) to be true "to first order" initially. Choquet-Bruhat [30] showed that this can be done precisely when the constraint equations (7)-(8) are satisfied with vanishing right hand side. Interestingly, to obtain existence for (13), Choquet-Bruhat's proof [30] does not in fact appeal to the techniques of Schauder [128], but, following Sobolev, rests on a Kirchhoff formula representation of the solution. Recently, new representations of this type have found applications to refined extension criteria [94].

An interesting feature of the classical existence and uniqueness proofs is that Theorem 2.3 requires more regularity than Theorem 2.2. This is because solutions of (11) are a priori only as regular as the metric. This difficulty has recently been overcome by Planchon and Rodnianski [121].

2.5. Stability of Minkowski space. The most celebrated global result on the Einstein equations is the stability of Minkowski space, first proven in monumental work of Christodoulou and Klainerman [46]:

THEOREM 2.5. Let $(\Sigma, \bar{g}, K)$ be a strongly asymptotically flat vacuum initial data set, assumed sufficiently close to Minkowski space in a weighted sense. Then the maximal development $(\mathcal{M}, g)$ is geodesically complete, and the spacetime approaches Minkowski space (with quantitative decay rates) in all directions. Moreover, a complete future null infinity $\mathcal{I}^{+}$can be attached to the spacetime as an ideal boundary such that $J^{-}\left(\mathcal{I}^{+}\right)=\mathcal{M}$.

The above theorem also allows one to rigorously define the laws of gravitational radiation. These laws are nonlinear even at infinity. Theorem 2.5 led to the discovery of Christodoulou's memory effect [37].

A new proof of a version of stability of Minkowski space using harmonic coordinates has been given by Lindblad and Rodnianski [104]. This has now been extended in various directions in [31]. The original result [46] was extended to the Maxwell case in the Ph.D. thesis of Zipser [147]. Bieri [11] has very recently given a proof of a version of stability of Minkowski space under weak asymptotics and regularity assumptions, following the basic setup of $[46]$.

There was an earlier semi-global result of Friedrich [75] where initial data were prescribed on a hyperboloidal initial hypersurface meeting $\mathcal{I}^{+}$.

A common misconception is that it is the positivity of mass which is somehow responsible for the stability of Minkowski space. The results of [104] for this are very telling, for they apply not only to the Einsteinvacuum equations, but also to the Einstein-scalar field system of Section 2.2.2, including the case where the definition of the energymomentum tensor (10) is replaced with its negative:

$$
T_{\mu \nu}=-\partial_{\mu} \psi \partial_{\nu} \psi+\frac{1}{2} g_{\mu \nu} \partial^{\alpha} \psi \partial_{\alpha} \psi
$$


In this latter case, Minkowski space is then not even a local minimizer for the mass functional in the class of perturbations allowed! Nonetheless, by the results of [104], Minkowski space is still stable in this context.

Stability of Minkowski space is the only truly global result on the maximal development which has been obtained for asymptotically flat initial data without symmetry. There are a number of important results applicable in cosmological settings, due to Friedrich [75], Andersson-Moncrief [3], and most recently Ringstrom [126].

Other than this, our current global understanding of solutions to the Einstein equations (in particular all work on the cosmic censorship conjectures) has been confined to solutions under symmetry. We refer the reader to the recent review article and book of Rendall $[\mathbf{1 2 4}, \mathbf{1 2 5}]$ for an overview and many references.

2.6. Penrose's incompleteness theorem. First a definition: Let $(\mathcal{M}, g)$ be a time-oriented Lorentzian manifold, and $S$ a closed spacelike 2 -surface. For any point $p \in S$, define two null mean curvatures tr $\chi$ and $\operatorname{tr} \bar{\chi}$, corresponding to the two future-directed null vectors $n(x), \bar{n}(x)$, where $n, \bar{n}$ are normal to $S$ at $x$. We say that $S$ is trapped if $\operatorname{tr} \chi<0, \operatorname{tr} \bar{\chi}<0$. Note that this definition does not depend on the choice of $n, \bar{n}$.

Theorem 2.6. (Penrose $1965[\mathbf{1 1 8}])$ Let $(\mathcal{M}, g)$ be globally hyperbolic ${ }^{9}$ with non-compact Cauchy surface $\Sigma$, where $g$ is a $C^{2}$ metric, and let

$$
R_{\mu \nu} V^{\mu} V^{\nu} \geq 0
$$

for all null vectors $V$. Then if $\mathcal{M}$ contains a closed trapped two-surface $S$, it follows that $(\mathcal{M}, g)$ is future causally geodesically incomplete.

This is the celebrated Penrose incompleteness theorem.

Note that solutions of the Einstein vacuum equations (3) satisfy (14). (Inequality (14), known as the null convergence condition, is also satisfied for solutions to the Einstein equations (1) coupled to most plausible matter models, specifically, if the energy momentum tensor $T_{\mu \nu}$ satisfies $T_{\mu \nu} V^{\mu} V^{\nu} \geq 0$ for all null $V^{\mu}$.) On the other hand, by definition, the maximal Cauchy development of initial data is globally hyperbolic (see Section 2.3). Thus, the theorem applies to the maximal development of (say) asymptotically flat vacuum initial data containing a trapped surface. By Cauchy stability $[\mathbf{8 1}]$, the presence of a trapped surface in $\mathcal{M}$ is clearly "stable" to perturbation of initial data.

Note finally that there are related incompleteness statements due to Penrose and Hawking [81] relevant in cosmological (see Section 4.8) settings.

The proof of Theorem 2.6 is a remarkable adaptation of classical comparison theorems of Riemannian geometry. Its simplicity, however, comes at a price. The theorem says nothing about the nature of incompleteness. Is incompleteness related to the curvature (say) blowing up along some or all

\footnotetext{
${ }^{9}$ See Appendix A.
} 
incomplete geodesics? In the asymptotically flat setting, is the incompleteness observable to a suitable class of "far-away" observers? These question will lead to Penrose's celebrated cosmic censorship conjectures. It is more natural to formulate these when we have some examples at our disposal. See Sections 3.1.5 and 3.2.3.

\section{Schwarzschild and Kerr}

This section shall introduce the geometry of the Schwarzschild and Kerr metrics, the most celebrated explicit solutions to the Einstein vacuum equations (3).

3.1. The Schwarzschild metric. We now readily associate the Schwarzschild metric with the black hole concept. It is important to remember, however, that the Schwarzschild solution was first discovered in a thoroughly classical astrophysical setting: it was to represent the vacuum region outside a star. The black hole interpretation-though in some sense inevitable-historically only emerged much later. Let us review briefly how this came about.

3.1.1. Schwarzschild's stars. The most basic self-gravitating objects are stars. In the most primitive stellar models, dating from the 19th century, stars are modeled by a self-gravitating fluid surrounded by vacuum. Moreover, to a first approximation, classically stars are spherically symmetric and static.

It should not be surprising then that early research on the Einstein equations (1) would address the question of the existence and structure of general relativistic stars in the new theory. In view of our above discussion, the most basic problem is to understand spherically symmetric, static metrics, represented in coordinates $(t, r, \theta, \phi)$, such that the spacetime has two regions: In the region $r \leq R_{0}$-the interior of the star-the metric should solve a suitable Einstein-matter system (1) with appropriate matter, and in the region $r \geq R_{0}$-the exterior of the star-the spacetime should be vacuum, i.e. the metric should solve (3).

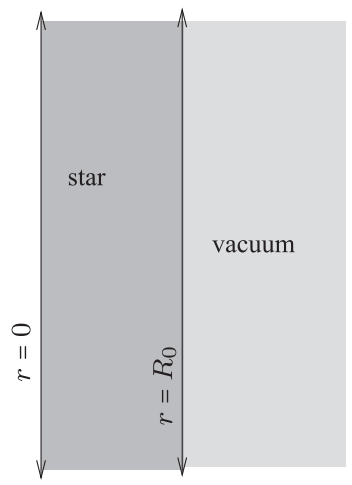


This is the problem first addressed by Schwarzschild $[\mathbf{1 3 1}, \mathbf{1 3 2}]$, already in 1916. Schwarzschild considered the vacuum region first [131] and arrived at the one-parameter family of solutions:

$$
g=-\left(1-\frac{2 M}{r}\right) d t^{2}+\left(1-\frac{2 M}{r}\right)^{-1} d r^{2}+r^{2}\left(d \theta^{2}+\sin ^{2} \theta d \phi^{2}\right) .
$$

Every student of this subject should explicitly check that this solves (3).

In [132], Schwarzschild found interior metrics for the darker shaded region $r \leq R_{0}$ above. In this region, matter is described by a perfect fluid. We shall not write down explicitly such metrics here, as this would require a long digression into fluids, their equations of state, etc. See [39]. Suffice it to say here that the existence of such solutions required that one take the constant $M$ positive, and the value $R_{0}$ marking the boundary of the star always satisfied $R_{0}>2 M$. The constant $M$ could then be identified with the total mass of the star as measured by considering the orbits of far-away test particles. ${ }^{10}$ In fact, for most reasonable matter models, static solutions of the type described above only exist under a stronger restriction on $R_{0}$ (namely $\left.R_{0} \geq 9 M / 4\right)$ now known as the Buchdahl inequality. See $[\mathbf{1 2}, \mathbf{2}, \mathbf{8 8}]$.

The restriction on $R_{0}$ necessary for the existence of Schwarzschild's stars appears quite fortuitous: It is manifest from the form (15) that the components of $g$ are singular if the $(t, r, \theta, \phi)$ coordinate system for the vacuum region is extended to $r=2 M$. But a natural question arises, namely, what happens if one does away completely with the star and tries simply to consider the expression (15) for all values of $r$ ? This at first glance would appear to be the problem of understanding the gravitational field of a "point particle" with the particle removed.

For much of the history of general relativity, the degeneration of the metric functions at $r=2 M$, when written in these coordinates, was understood as meaning that the gravitational field should be considered singular there. This was the famous Schwarzschild "singularity". ${ }^{11}$ Since "singularities" were considered "bad" by most pioneers of the theory, various arguments were concocted to show that the behaviour of $g$ where $r=2 M$ is to be thought of as "pathological", "unstable", "unphysical" and thus, the solution should not be considered there. The constraint on $R_{0}$ related to the Buchdahl inequality seemed to give support to this point of view. See also $[\mathbf{7 0}]$.

3.1.2. The maximal extension of Synge and Kruskal. It turns out that the above point of view was incorrect, essentially at every level. Let us for now, however, avoid the question of the physical interpretation of such pure vacuum solutions with the star removed, and simply ask the purely mathematical question of how big can be the underlying manifold on which such a

\footnotetext{
${ }^{10}$ Test particles in general relativity follow timelike geodesics of the spacetime metric.

${ }^{11}$ Let the reader keep in mind that there is a good reason for the quotation marks here and for those that follow.
} 
solution lives. This leads to the notion of a "maximally extended" solution. In the case of Schwarzschild, this will be a spacetime which, although not to be taken as a model for anything per se, can serve as a reference for the formulation of all important concepts in the subject.

Synge was the first to consider these issues systematically and construct "maximal extensions" of the original Schwarzschild metric in a paper [136] of 1950. A more concise approach to such a construction was given in a celebrated 1960 paper [98] of Kruskal. Indeed, let $\mathcal{M}$ be the manifold with differentiable structure given by $\mathcal{U} \times \mathbb{S}^{2}$ where $\mathcal{U}$ is the open subset $T^{2}-R^{2}<1$ of the $(T, R)$-plane. Consider the metric $g$

$$
g=\frac{32 M^{3}}{r} e^{-r / 2 M}\left(-d T^{2}+d R^{2}\right)+r^{2} d \sigma_{\mathbb{S}}^{2}
$$

where $r$ is defined implicitly by

$$
T^{2}-R^{2}=\left(1-\frac{r}{2 M}\right) e^{r / 2 M} .
$$

The region $\mathcal{U}$ is depicted below:

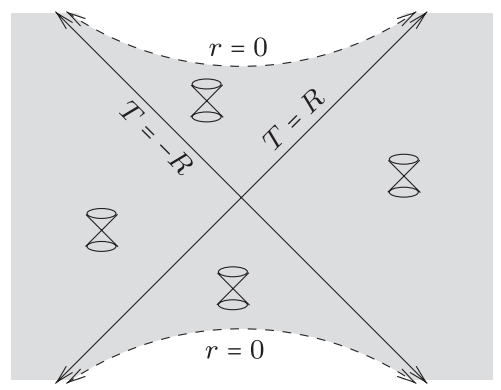

This is a spherically symmetric 4-dimensional Lorentzian manifold satisfying (3) such that the original Schwarzschild metric is isometric to the region $R>|T|$ (where $t$ is given by $\tanh \left(\frac{t}{4 M}\right)=T / R$ ). It can be shown now that $(\mathcal{M}, g)$ is inextendible as a $C^{2}$ (in fact $C^{0}$ ) Lorentzian manifold, that is to say, if

$$
i:(\mathcal{M}, g) \rightarrow(\widetilde{\mathcal{M}}, \tilde{g})
$$

is an isometric embedding, where $(\widetilde{M}, \tilde{g})$ is a $C^{2}$ (in fact $\left.C^{0}\right)$ 4-dimensional Lorentzian manifold, then necessarily $i(\mathcal{M})=\widetilde{\mathcal{M}}$.

The above property defines the sense in which our spacetime is "maximally" extended, and thus, $(\mathcal{M}, g)$ is called sometimes maximally-extended Schwarzschild. In later sections, we will often just call it "the Schwarzschild solution".

Note that the form of the metric is such that the light cones are as depicted. Thus, one can read off much of the causal structure by sight.

It may come as a surprise that in maximally-extended Schwarzschild, there are two regions which are isometric to the original $r>2 M$ Schwarzschild region. Alternatively, a Cauchy surface will have topology $\mathbb{S}^{2} \times \mathbb{R}$ with two asymptotically flat ends. This suggests that this spacetime 
is not to be taken as a physical model. We will discuss this later on. For now, let us simply try to understand better the global geometry of the metric.

3.1.3. The Penrose diagram of Schwarzschild. There is an even more useful way to represent the above spacetime. First, let us define null co ordinates $U=T-R, V=T+R$. These coordinates have infinite range. We may rescale them by $u=u(U), v=v(V)$ to have finite range. (Note the freedom in the choice of $u$ and $v !)$ The domain of $(u, v)$ coordinates, when represented in the plane where the axes are at 45 and 135 degrees with the horizontal, is known as a Penrose diagram of Schwarzschild. Such a Penrose diagram is depicted below ${ }^{12}$ :

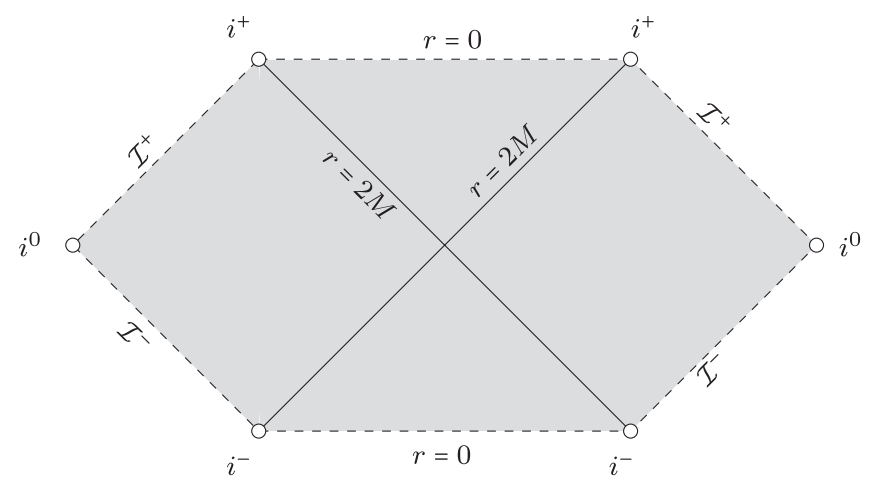

In more geometric language, one says that a Penrose diagram corresponds to the image of a bounded conformal map

$$
\mathcal{M} / \mathrm{SO}(3)=\mathcal{Q} \rightarrow \mathbb{R}^{1+1}
$$

where one makes the identification $v=t+x, u=t-x$ where $(t, x)$ are now the standard coordinates $\mathbb{R}^{1+1}$ represented in the standard way on the plane. We further assume that the map preserves the time orientation, where Minkowski space is oriented by $\partial_{t}$. (In our application, this is a fancy way of saying that $\left.u^{\prime}(U), v^{\prime}(V)>0\right)$. It follows that the map preserves the causal structure of $\mathcal{Q}$. In particular, we can "read off" the radial null geodesics of $\mathcal{M}$ from the depiction.

Now we may turn to the boundary induced by the causal embedding. We define $\mathcal{I}^{ \pm}$to be the boundary components as depicted. ${ }^{13}$ These are characterized geometrically as follows: $\mathcal{I}^{+}$are limit points of future-directed null rays in $\mathcal{Q}$ along which $r \rightarrow \infty$. Similarly, $\mathcal{I}^{-}$are limit points of pastdirected null rays for which $r \rightarrow \infty$. We call $\mathcal{I}^{+}$future null infinity and $\mathcal{I}^{-}$ past null infinity. The remaining boundary components $i^{0}$ and $i^{ \pm}$depicted are often given the names spacelike infinity and future (past) timelike infinity, respectively.

\footnotetext{
${ }^{12}$ Note that $(u, v)$ can indeed be chosen so that the $r=0$ boundaries are horizontal lines as depicted.

${ }^{13}$ Our convention is that open endpoint circles are not contained in the intervals they bound, and dotted lines are not contained in the regions they bound, whereas solid lines are.
} 
In the physical application, it is important to remember that asymptotically flat ${ }^{14}$ spacetimes like our $(\mathcal{M}, g)$ are not meant to represent the whole universe ${ }^{15}$ but rather, the gravitational field in the vicinity of an isolated self-gravitating system. $\mathcal{I}^{+}$is an idealization of far away observers who can receive radiation from the system. In this sense, "we"-as astrophysical observers of stellar collapse, say-are located at $\mathcal{I}^{+}$. The ambient causal structure of $\mathbb{R}^{1+1}$ allows us to talk about $J^{-}(p) \cap \mathcal{Q}$ for $p \in \mathcal{I}^{+}$. ${ }^{16}$ And this will lead us to the black hole concept. Therein lies the use of the Penrose diagram representation.

The systematic use of the conformal point of view to represent the global geometry of spacetimes is one of the many great contributions of Penrose to general relativity. These representations can be traced back to the wellknown "spacetime diagrams" of special relativity, promoted especially by Synge [137]. The "formal" use of Penrose diagrams in the sense above goes back to Carter [25], in whose hands these diagrams became a powerful tool for determining the global structure of all classical black hole spacetimes. It is hard to overemphasize how important it is for the student of this subject to become comfortable with these representations.

3.1.4. The black hole concept. With Penrose diagram notation, one can now understand the black hole concept. First an important remark: In Schwarzschild, the boundary component $\mathcal{I}^{+}$enjoys a limiting affine completeness. More specifically, normalising a sequence of ingoing radial null vectors by parallel transport along an outgoing geodesic meeting $\mathcal{I}^{+}$, the affine length of the null geodesics generated by these vectors, parametrized by their parallel transport (restricted to $J^{-}\left(\mathcal{I}^{+}\right)$), tends to infinity:

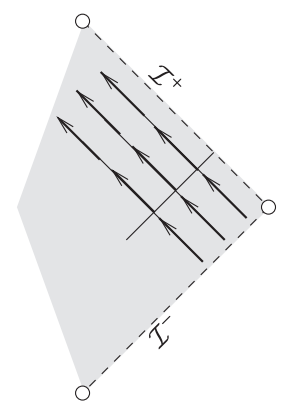

This has the interpretation that far-away observers in the radiation zone can observe for all time. (This is in some sense related to the presence of timelike geodesics near infinity of infinite length, but the completeness is best formulated with respect to $\mathcal{I}^{+}$.) A similar statement clearly holds for $\mathcal{I}^{-}$.

Given this completeness property, let us define now the black hole region to be $\mathcal{Q} \backslash J^{-}\left(\mathcal{I}^{+}\right)$, and the white hole region to be $\mathcal{Q} \backslash J^{+}\left(\mathcal{I}^{-}\right)$. Thus, the

\footnotetext{
${ }^{14}$ See Appendix 2.2.3 for a definition.

${ }^{15}$ The study of that problem is what is known as "cosmology". See Section 4.8.

${ }^{16}$ Refer to Appendix A for $J^{ \pm}$.
} 
black hole corresponds to those points of spacetime which cannot "send signals" to future null infinity, or, in the physical interpetation, to far-away observers who (in view of the completeness property!) nonetheless can observe radiation for infinite time.

The future boundary of $J^{-}\left(\mathcal{I}^{+}\right)$in $\mathcal{Q}$ (alternatively characterized as the past boundary of the black hole region) is a null hypersurface known as the future event horizon, and is denoted by $\mathcal{H}^{+}$. Exchanging past and future, we obtain the past event horizon $\mathcal{H}^{-}$. In maximal Schwarzschild, $\{r=2 M\}=\mathcal{H}^{+} \cup \mathcal{H}^{-}$. The subset $J^{-}\left(\mathcal{I}^{+}\right) \cap J^{+}\left(\mathcal{I}^{-}\right)$is known as the domain of outer communications.

Let us consider at the case of Minkowski space for comparison. Note that this is again described by (15) but with $M=0$. Here, $\mathcal{Q}=\mathbb{R}^{3+1} / \mathrm{SO}(3)$ is a manifold with boundary since the $\mathrm{SO}(3)$ action has a locus of fixed points, the centre of symmetry. A Penrose diagram of Minkowski space is easily seen to be:

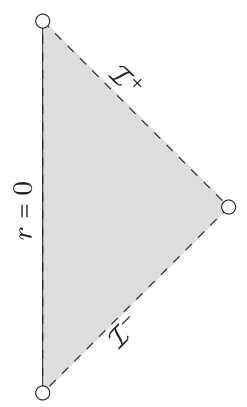

Here $\mathcal{I}^{+}$and $\mathcal{I}^{-}$are characterized as before, and enjoy the same completeness property as in Schwarzschild. One reads off immediately that $J^{-}\left(\mathcal{I}^{+}\right) \cap \mathcal{Q}=\mathcal{Q}$, i.e. $\mathbb{R}^{3+1}$ does not contain a black hole under the above definitions.

It was mentioned before that maximal Schwarzschild is not to be considered "physical". The simplest "physical" model of a black hole spacetime are provided by the celebrated Oppenheimer-Snyder solutions [117]. Having now the notation of Penrose diagrams, one can concisely describe their geometry without giving explicit forms of the metric. Like Schwarzschild's original picture of the gravitational field of a spherically symmetric star, these solutions involve a region $r \leq R_{0}$ solving (1) and $r \geq R_{0}$ satisfying (3). The matter is described now by a pressureless fluid which is initially assumed homogeneous in addition to being spherically symmetric. The assumption of staticity is however dropped, and for appropriate initial conditions, it follows that $R_{0}\left(t^{*}\right) \rightarrow 0$ with respect to a suitable time coordinate $t^{*}$. (In fact, the Einstein equations can be reduced to an o.d.e. for $R_{0}\left(t^{*}\right)$.) One says that the star "collapses". ${ }^{17}$ A Penrose diagram of such a solution

\footnotetext{
${ }^{17}$ Note that $R_{0}\left(t^{*}\right) \rightarrow 0$ does not mean that the star collapses to "a point", merely that the spheres which foliate the interior of the star shrink to 0 area. The limiting singular boundary is a spacelike hypersurface as depicted.
} 
(to the future of a Cauchy hypersurface) can be seen to be of the form:

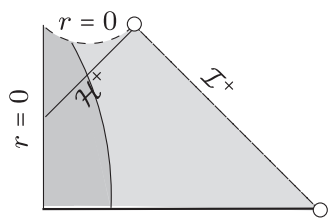

The lighter shaded region is isometric to a subset of maximal Schwarzschild. In particular, the completeness property of $\mathcal{I}^{+}$holds, and as before, we identify the black hole region to be $\mathcal{Q} \backslash J^{-}\left(\mathcal{I}^{+}\right)$.

In contrast to maximal Schwarzschild, where the initial configuration is unphysical (the Cauchy surface has two ends and topology $\mathbb{R} \times \mathbb{S}^{2}$ ), here the initial configuration is entirely plausible: the Cauchy surface is topologically $\mathbb{R}^{3}$, and its geometry is not far from Euclidean space.

It is traditional in general relativity to "think" Oppenheimer-Snyder but "write" maximally-extended Schwarzschild. In particular, one often imports terminology like "collapse" in discussing Schwarzschild, and one often reformulates our definitions replacing $\mathcal{I}^{+}$with one of its connected components, that is to say, we will often write $J^{-}\left(\mathcal{I}^{+}\right) \cap J^{+}\left(\mathcal{I}^{+}\right)$meaning $J^{-}\left(\mathcal{I}_{A}^{+}\right) \cap J^{+}\left(\mathcal{I}_{A}^{-}\right)$, etc. In any case, the precise relation between the two solutions should be clear from the above discussion. In view of Cauchy stability results $[\mathbf{8 1}]$, sufficiently general theorems about the Cauchy problem on maximal Schwarzschild lead immediately to such results on OppenheimerSnyder. One should always keep this relation in mind.

The above definition of black hole for the Schwarzschild and Oppenheimer-Snyder metrics should be thought of as a blueprint for how to define the notion of black hole region in general. That is to say, to define the black hole region, one needs

(1) some notion of future null infinity $\mathcal{I}^{+}$,

(2) a way of identifying $J^{-}\left(\mathcal{I}^{+}\right)$, and

(3) some characterization of the "completeness" of $\mathcal{I}^{+} .18$

If $\mathcal{I}^{+}$is indeed complete, we can define the black hole region as

"the complement in $\mathcal{M}$ of $J^{-}\left(\mathcal{I}^{+}\right)$".

For spherically symmetric spacetimes arising as solutions of the Cauchy problem for (1), one can show that there always exists a Penrose diagram, and thus, a definition can be formalised along precisely these lines (see [53]).

${ }^{18}$ The characterization of completeness can be formulated for general asymptotically flat vacuum space times using the results of $[46]$. This formulation is due to Christodoulou [42]. Previous attempts to formalise these notions rested on "asymptotic simplicity" and "weak asymptotic simplicity". See [81]. Although the qualitative picture suggested by these notions appears plausible, the detailed asymptotic behaviour of solutions to the Einstein equations turns out to be much more subtle, and Christodoulou has proven $[43]$ that these notions cannot capture even the simplest generic physically interesting systems. 
For spacetimes without symmetry, however, even defining the relevant asymptotic structure so that this structure is compatible with the theorems one is to prove is a main part of the problem. This has been accomplished only in the case of perturbations of Minkowski space. In particular, as we have seen in Section 2.5, Christodoulou and Klainerman [46] have shown that spacetimes arising from perturbations of Minkowski initial data have a complete $\mathcal{I}^{+}$in a well defined sense, whose past can be identified and is indeed the whole spacetime. That is to say, small perturbations of Minkowski space cannot form black holes.

3.1.5. Negative mass Schwarzschild and weak cosmic censorship. Schwarzschild's stars required taking $M>0$. But what happens when one takes $M<0$ in (15)? This defines so-called negative mass Schwarzschild. The metric element (15) for such $M$ is now regular for all $r>0$. The limiting singular behaviour of the metric at $r=0$ is in fact essential, i.e. one can show that along inextendible incomplete geodesics the curvature blows up. Thus, one immediately arrives at a maximally extended solution which can be seen to have Penrose diagram:

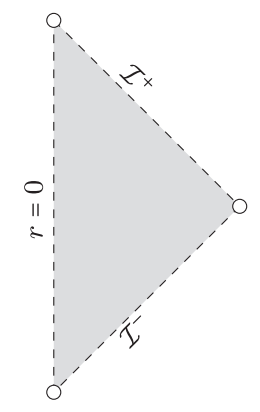

Note that in contrast to the case of $\mathbb{R}^{3+1}$, the boundary $r=0$ is here depicted by a dotted line denoting (according to our conventions) that it is not part of $\mathcal{Q}$ !

The above spacetime is interpreted as having a "naked singularity". The traditional way of describing this in the physics literature is to remark that the "singularity" $\mathcal{B}=\{r=0\}$ is "visible" to $\mathcal{I}^{+}$, i.e., $J^{-}\left(\mathcal{I}^{+}\right) \cap \mathcal{B} \neq \varnothing$. From the point of view of the Cauchy problem, however, this characterization is meaningless because the above maximal extension is not globally hyperbolic, i.e. it is not uniquely characterized by an appropriate notion of initial data. ${ }^{19}$ From the point of view of the Cauchy problem, one must not consider maximal extensions but the maximal Cauchy development of initial data, which by definition is globally hyperbolic (see Theorem 2.4 of Appendix 2). Considering an inextendible spacelike hypersurface $\Sigma$ as a Cauchy surface, the maximal Cauchy development of $\Sigma$ would be the darker shaded

\footnotetext{
${ }^{19}$ See Appendix A for the definition of global hyperbolicity.
} 
region depicted below:

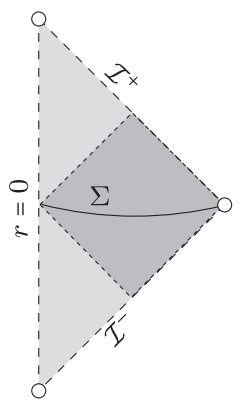

The proper characterization of "having a naked singularity", from the point of view of the darker shaded spacetime, is that its $\mathcal{I}^{+}$is incomplete. Let us take this as a definition. Of course, this example does not say anything about the dynamic formation of naked singularities, because the inital data hypersurface $\Sigma$ is already in some sense "singular", for instance, it is geodesically incomplete, and the curvature blows up along incomplete geodesics. The dynamic formation of a naked singularity from regular, complete initial data would be pictured by:

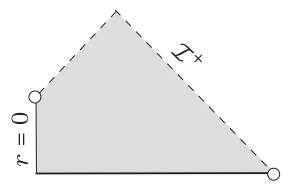

where we are to understand also in the above that $\mathcal{I}^{+}$is incomplete. We have

Conjecture. (Weak cosmic censorship) For generic asympotically flat ${ }^{20}$ initial data for "reasonable" Einstein-matter systems, the maximal Cauchy development "possesses a complete $\mathcal{I}^{+} "{ }^{21}$

Christodoulou $[\mathbf{4 0 , 4 2}]$ has shown this conjecture to be true for the Einstein-scalar field system under spherical symmetry. On the other hand, he has also shown $[\mathbf{3 8}]$ that the assumption of genericity is necessary by explicitly constructing solutions with incomplete $\mathcal{I}^{+}$and Penrose diagram as depicted above.

In light of the above conjecture, the story of the Oppenheimer-Snyder solution and its role in the emergence of the black hole concept does have an interesting epilogue, however. Recall that in the Oppenheimer-Snyder solutions, the region $r \leq R_{0}$, in addition to being spherically symmetric, is homogeneous. It turns out that by considering spherically symmetric initial data for which the "star" is no longer homogeneous, Christodoulou has proven that one can arrive at spacetimes for which "naked singularities" form as in the above Penrose diagram [34]. Moreover, it is shown in [34] that

\footnotetext{
${ }^{20}$ See Section 2.2.3 for a formulation of this notion. Asymptotically flat implies in particular complete.

${ }^{21}$ This conjecture is originally due to Penrose [119]. The present formulation is taken from Christodoulou [42].
} 
this occurs for an open subset of initial data within spherical symmetry. Thus, the statement of weak cosmic censorship is violated for this matter model when the conjecture is restricted to spherically symmetric data. The fact that in the Oppenheimer-Snyder solutions black holes formed appears thus to be a rather fortuitous accident! Nonetheless, we should note that the failure of weak cosmic censorship in this context is believed to be due to the inappropriateness of the pressureless model, not as indicative of actual phenomena. Hence, the restriction on the matter model to be "reasonable" in the formulation of the weak cosmic censorship conjecture.

3.2. The Kerr metric. What about other vacuum solutions?

We have the so-called Birkhoff's theorem:

TheOREM 3.1. Let $(\mathcal{M}, g)$ be a spherically symmetric solution to the vacuum equations (3). Then it is locally isometric to a Schwarzschild solution with parameter $M$, for some $M \in \mathbb{R}$.

Thus one must look for solutions with less symmetry. It was not until 1963 that Roy Kerr discovered that the Schwarzschild family is a subfamily of a two parameter family of axisymmetric stationary vacuum metrics now known as the Kerr family.

3.2.1. Boyer-Lindquist coordinates. The Kerr metric is a 2-parameter family of vacuum metrics first discovered [90] in 1963. The parameters are called mass $M$ and specific angular momentum $a$, i.e. angular momentum per unit mass. In so-called Boyer-Lindquist local coordinates, the metric element takes the form:

$$
\begin{aligned}
& -\left(1-\frac{2 M}{r\left(1+\frac{a^{2} \cos ^{2} \theta}{r^{2}}\right)}\right) d t^{2}+\frac{1+\frac{a^{2} \cos ^{2} \theta}{r^{2}}}{1-\frac{2 M}{r}+\frac{a^{2}}{r^{2}}} d r^{2}+r^{2}\left(1+\frac{a^{2} \cos ^{2} \theta}{r^{2}}\right) d \theta^{2} \\
& +r^{2}\left(1+\frac{a^{2}}{r^{2}}+\left(\frac{2 M}{r}\right) \frac{a^{2} \sin ^{2} \theta}{r^{2}\left(1+\frac{a^{2} \cos ^{2} \theta}{r^{2}}\right)}\right) \sin ^{2} \theta d \phi^{2} \\
& -4 M \frac{a \sin ^{2} \theta}{r\left(1+\frac{a^{2} \cos ^{2} \theta}{r^{2}}\right)} d t d \phi .
\end{aligned}
$$

The vector fields $\partial_{t}$ and $\partial_{\phi}$ are Killing. We say that the Kerr family is stationary and axisymmetric. ${ }^{22}$ Traditionally, one denotes

$$
\Delta=r^{2}-2 M r+a^{2} .
$$

If $a=0$, the Kerr metric clearly reduces to Schwarzschild (15).

Maximal extensions of the Kerr metric were first constructed by Carter [26]. For parameter range $0 \leq|a|<M$, these maximal extensions have black hole regions and white hole regions bounded by future and past event

\footnotetext{
${ }^{22}$ There are various conventions on the meaning of the words "stationary" and "axisymmetric" depending on the context. Let us not worry about this here...
} 
horizons $\mathcal{H}^{ \pm}$meeting at a bifurcate sphere. The above coordinate system is defined in a domain of outer communications, and the horizon will correspond to the limit $r \rightarrow r_{+}$, where $r_{+}$is the larger positive root of $\Delta=0$, i.e.

$$
r_{+}=M+\sqrt{M^{2}-a^{2}} .
$$

Since the motivation of our study is the Cauchy problem for the Einstein equations, it is more natural to consider not maximal extensions, but maximal developments of complete initial data. (See Section 2.) In the Schwarzschild case, the maximal development of initial data on a Cauchy surface $\Sigma$ as described previously coincides with maximally-extended Schwarzschild. In Kerr, if we are to take an asymptotically flat (with two ends) hypersurface in a maximally extended Kerr for parameter range $0<|a|<M$, then its maximal development will have a smooth boundary in maximally-extended Kerr. This boundary is what is known as a Cauchy horizon. We have already discussed this phenomenon in Section 3.2.3 in the context of strong cosmic censorship. The maximally extended Kerr solutions are quite bizarre, in particular, they contain closed timelike curves. This is of no concern to us here, however. By definition, for us the term "Kerr metric $\left(\mathcal{M}, g_{M, a}\right)$ " will always denote the maximal development of a complete asymptotically flat hypersurface $\Sigma$, as above, with two ends. One can depict the Penrosediagramatic representation of a suitable two-dimensional timelike slice of this solution as below:

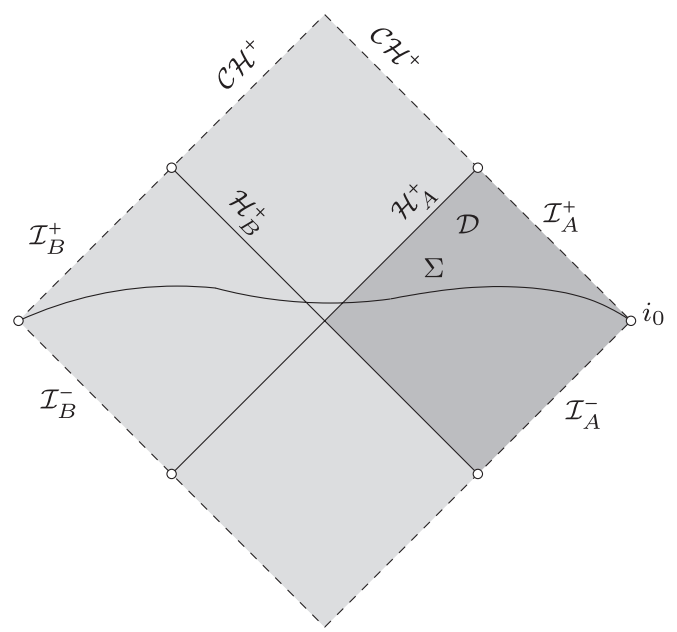

With this convention in mind, we note that the dependence of $g_{M, a}$ on $a$ is smooth in the range $0 \leq|a|<M$. In particular, Kerr solutions with small $|a| \ll M$ can be viewed as close to Schwarzschild.

One can see this explicitly in the subregion of interest to us by passing to a new system of coordinates. Define

$$
\begin{aligned}
t^{*} & =t+\bar{t}(r) \\
\phi^{*} & =\phi+\bar{\phi}(r)
\end{aligned}
$$


where

$$
\frac{d \bar{t}}{d r}(r)=\left(r^{2}+a^{2}\right) / \Delta^{2} . \quad \frac{d \bar{\phi}}{d r}(r)=a / \Delta .
$$

(These coordinates are often known as Kerr-star coordinates.) These coordinates are regular across $\mathcal{H}^{+} \backslash \mathcal{H}^{-} .{ }^{23}$ We may finally define a coordinate $r_{\text {Schw }}=r_{\text {Schw }}(r, a)$ such that which takes $\left[r_{+}, \infty\right) \rightarrow[2 M, \infty)$ with smooth dependence in $a$. In particular, if we define $\Sigma_{0}$ by $\mathcal{D}=\left\{t^{*}=0\right\}$, and define $\mathcal{R}=\mathcal{D} \cap\left\{t^{*} \geq 0\right\}$, and fix $r_{\mathrm{Schw}}, t^{*}, \phi^{*}$ Schwarzschild coordinates, then the metric functions of $g_{M, a}$ written in terms of these coordinates as defined previously depend smoothly on $a$ for $0 \leq|a|<M$.

3.2.2. The ergoregion. We note that $\partial_{t}=\partial_{t^{*}}$ in the intersection of the coordinate systems. We immediately note that $\partial_{t}$ is spacelike on the horizon, except where $\theta=0, \pi$, i.e. on the axis of symmetry. Note that we shall often abuse notation (as we just have done) and speak of $\partial_{t}$ on the horizon or at $\theta=0$, where of course the $(r, t, \theta, \phi)$ coordinate system breaks down, and formally, this notation is meaningless.

In general, the part of the domain of outer communications plus horizon where $\partial_{t}$ is spacelike is known as the ergoregion. It is bounded by a hypersurface known as the ergosphere. The ergosphere meets the horizon on the axis of symmetry $\theta=0, \pi$.

The ergosphere allows for a particle "process", originally discovered by Penrose [119], for extracting energy out of a black hole. This came to be known as the Penrose process. In his thesis, Christodoulou [33] discovered the existence of a quantity-the so-called irreducible mass of the black holewhich he showed to be always nondecreasing in a Penrose process. The analogy between this quantity and entropy led later to a subject known as "black hole thermodynamics" $[\mathbf{8}, \mathbf{1 0}]$. This is currently the subject of intense investigation from the point of view of high energy physics.

The presence of the ergoregion will be one of the fundamental difficulties for understanding the stability problem in Section 4.

3.2.3. The Cauchy horizon and strong cosmic censorship. Following [44], we have called Theorem 2.6 an "incompleteness theorem" and not a "singularity theorem" as is more standard. The reason is precisely the situation illustrated by Kerr. Note that the Kerr solution satisfies the assumptions of Theorem 2.6 and thus is future causally geodesically incomplete. The maximal development is however extendible as a $C^{\infty}$ Lorentzian manifold such that every incomplete causal geodesic of the original spacetime enter the extension! Thus, in the context of Kerr initial data, the statement of Theorem 2.6 does not result in local breakdown of the metric, but rather in

\footnotetext{
${ }^{23}$ Of course, one again needs two coordinate systems in view of the breakdown of spherical coordinates. We shall suppress this issue in the discussion that follows.
} 
the breakdown of globally hyperbolicity itself, and thus predictability of the future from initial data. ${ }^{24}$

The boundary of $(\mathcal{M}, g)$ in such extensions corresponds to $\mathcal{C H}^{+}$above. This boundary is known as the Cauchy horizon. From the philosophical point of view, the existence of Cauchy horizons is quite problematic. Observers crossing them are not destroyed, yet the theory can no longer predict their future. The purpose of the strong cosmic censorship conjecture is to protect the theory from this predicament:

Conjecture. (Strong cosmic censorship) The maximal development of generic asymptotically flat initial data for the vacuum Einstein equations is inextendible as a suitably regular Lorentzian metric. ${ }^{25}$

One can paraphrase this conjecture as saying that whenever one has geodesic incompleteness, it is due to a local breakdown of the metric. In view of the above comments, for this conjecture to be true, the behaviour of the Kerr metric described above would have to be unstable to perturbation. ${ }^{26}$ Thus, if by the term "singularity" one wants to suggest "breakdown of the metric", it is only a positive resolution of the strong cosmic censorship conjecture that would in particular (generically) make Theorem 2.6 into a true "singularity theorem".

Finally, let us note that the question of what is the proper notion of inextendibility in the formulation of the above conjecture may be a subtle one. A spherically symmetric analogue of the behaviour exhibited by Kerr is given by the so-called Reissner-Nordström metric, which is a solution to the Einstein-Maxwell equations with metric given in local coordinates by

$$
-\left(1-2 M / r+e^{2} / r^{2}\right) d t^{2}+\left(1-2 M / r+e^{2} / r^{2}\right)^{-1} d r^{2}+r^{2}\left(d \theta^{2}+\sin ^{2} \theta d \phi^{2}\right),
$$

and global structure given precisely by the Penrose diagram of the previous page. In particular, as in the Kerr metric, this spacetime can be extended to a larger spacetime across a smooth Cauchy horizon. One can study the

\footnotetext{
${ }^{24}$ Further confusion can arise from the fact that "maximal extensions" of Kerr constructed with the help of analyticity are still geodesically incomplete and inextendible, in particular, with the curvature blowing up along all incomplete causal geodesics. Thus, one often talks of the "singularities" of Kerr, referring to the ideal singular boundaries one can attach to such extensions. One must remember, however, that these extensions are of no relevance from the point of view of the Cauchy problem, and in any case, their singular behaviour in principle has nothing to do with Theorem 2.6.

${ }^{25}$ As with weak cosmic censorship, the original formulation of this conjecture is due to Penrose [120]. The formulation given here is from [42]. Related formulations are given in $[49,110]$. One can also pose the conjecture for compact initial data, and for various Einstein-matter systems. It should be emphasized that "strong cosmic censorship" does not imply "weak cosmic censorship". For instance, one can imagine a spacetime with Penrose diagram as in the last diagram before the formulation of the latter conjecture, with incomplete $\mathcal{I}^{+}$, but still inextendible across the null "boundary" emerging from the centre.

${ }^{26}$ Note that the instability concerns a region "far inside" the black hole interior. The black hole exterior is expected to be stable as in the formulation of Section 4.1.
} 
stability of the Reissner-Nordström Cauchy horizon by viewing it as a special solution of the Einstein-Maxwell-scalar field system: (1),

$$
T_{\mu \nu}=\frac{1}{4 \pi}\left(F_{\mu \lambda} F_{\nu \rho} g^{\lambda \rho}-\frac{1}{4} g_{\mu \nu} F_{\alpha \beta} F_{\gamma \delta} g^{\alpha \gamma} g^{\beta \delta}\right)+\partial_{\mu} \psi \partial_{\nu} \psi-g_{\mu \nu} g^{\alpha \beta} \partial_{\alpha} \psi \partial_{\beta} \psi
$$

where $\psi=0$. We have in particular $[\mathbf{5 1}, \mathbf{5 2}]$

TheOREM 3.2. For the system (1), (16), (17) restricted to spherical symmetry, there exists an open set of asymptotically flat initial data such that the causal structure of the spherically symmetric quotient $\mathcal{Q}$ of the maximal development coincides with that of Reissner-Nordstrom in a neighborhood of the future limit point of $\mathcal{I}^{+}$. Moreover, the maximal development is extendible as a $C^{0}$ Lorentzian metric.

Thus, the formulation of strong cosmic censorship of [42] does not hold in this context. It is shown in addition [52] that under a plausible assumption on the behaviour of $\psi$ on $\mathcal{H}^{+}$, the above extensions cannot be $C^{2}$. Thus, a weaker formulation of strong cosmic censorship may still hold.

\section{The black hole stability problem}

4.1. The nonlinear stability of Kerr conjecture. The central open problem in this direction is the nonlinear stability of the Kerr family. Let us give here a rough formulation.

Conjecture. (Nonlinear stability of Kerr) Let $(\Sigma, \bar{g}, K)$ be a vacuum initial data set (see Section 2.2) sufficiently close (in a weighted sense) to the initial data on a Cauchy hypersurface in the Kerr solution $\left(\mathcal{M}, g_{M, a}\right)$ for some parameters $0 \leq|a|<M$. Then the maximal vacuum development $(\mathcal{M}, g)$ possesses a complete null infinity $\mathcal{I}^{+}$such that the metric restricted to $J^{-}\left(\mathcal{I}^{+}\right)$approaches a Kerr solution $\left(\mathcal{M}, g_{M_{f}, a_{f}}\right)$ in a uniform way with quantitative decay rates, where $M_{f}, a_{f}$ are near $M$, a respectively.

Let us make some remarks concerning the above statement. Under the assumptions of the above conjecture, $(\mathcal{M}, g)$ certainly contains a trapped surface $S$ by Cauchy stability [81]. By Penrose's incompleteness theorem (Theorem 2.6), this implies that $(\mathcal{M}, g)$ is future causally geodesically incomplete. By the methods of the proof of Theorem 2.6, it is easy to see that $S \cap J^{-}\left(\mathcal{I}^{+}\right)=\varnothing$. Thus, as soon as $\mathcal{I}^{+}$is shown to be complete, it would follow that the spacetime has a black hole region. ${ }^{27}$

In the spherically symmetric analogue of this problem where the Einstein equations are coupled with matter, or the 5-dimensional triaxial Bianchi IX vacuum problem discussed in Section 5 , the completeness of null infinity can

\footnotetext{
${ }^{27}$ Let us also remark the obvious fact that the above conjecture implies in particular that weak cosmic censorship holds in a neighborhood of Kerr data.
} 
be inferred easily without detailed understanding of the geometry $[\mathbf{5 3}, \mathbf{5 6}]$. One can view this as an "orbital stability" statement. In this spherically symmetric case, the asymptotic stability can then be studied a posteriori, as in $[\mathbf{5 7}, \mathbf{8 4}]$. This latter problem is much more difficult.

In the case of Conjecture 4.1, in contrast to the symmetric cases mentioned above, one does not expect to be able to show any weaker stability statement than that of asymptotic stability with decay rates, as stated above. Note that it is only the Kerr family as a whole- - ot the Schwarzschild subfamily-which is expected to be asymptotically stable: Choosing $a=0$ certainly does not imply that $a_{f}=0$. On the other hand, if $|a| \ll M$, then by the formulation of the above conjecture, it would follow that $\left|a_{f}\right| \ll M_{f}$. It is with this in mind that we will consider $|a| \ll M$ case later in this article.

4.2. Introduction to $\square_{g} \boldsymbol{\psi}=\mathbf{0}$. In the remainder of this article, we will concern ourselves solely with linear wave equations on black hole backgrounds, specifically, the scalar linear homogeneous wave equation

$$
\square_{g} \psi=0 \text {. }
$$

The study of the solutions to such equations is motivated by the stability problem for the black hole spacetimes themselves as solutions to (3). The equation (18) can be viewed as a poor man's linearisation of (3), neglecting tensorial structure. Other linear problems with an even closer relationship to the study of the Einstein equations will be discussed in Section 5 .

Proposition 4.2.1. Let $(\mathcal{M}, g)$ be globally hyperbolic and $\Sigma$ a Cauchy hypersurface. If $\psi \in H_{\mathrm{loc}}^{2}(\Sigma), \psi^{\prime} \in H_{\mathrm{loc}}^{1}(\Sigma)$, then there is a unique $\psi$ with $\left.\psi\right|_{\mathcal{S}} \in H_{\mathrm{loc}}^{2}(\mathcal{S}),\left.n_{\mathcal{S}} \psi\right|_{\mathcal{S}} \in H_{\mathrm{loc}}^{1}(\mathcal{S})$, for all spacelike $\mathcal{S} \subset \mathcal{M}$, satisfying

$$
\square_{g} \psi=0,\left.\quad \psi\right|_{\Sigma}=\psi,\left.\quad n_{\Sigma} \psi\right|_{\Sigma}=\psi^{\prime},
$$

where $n_{\Sigma}$ denotes the future unit normal of $\Sigma$. For $m \geq 1$, if $\psi \in H_{\mathrm{loc}}^{m+1}$, $\psi^{\prime} \in H_{\mathrm{loc}}^{m}$, then $\left.\psi\right|_{\mathcal{S}} \in H_{\mathrm{loc}}^{m+1}(\mathcal{S}),\left.n_{\mathcal{S}} \psi\right|_{\mathcal{S}} \in H_{\mathrm{loc}}^{m}(\mathcal{S})$. Moreover, if $\psi_{1}, \psi_{1}^{\prime}$, and $\psi_{2}, \psi_{2}^{\prime}$ are as above and $\psi_{1}=\psi_{2}, \psi_{1}^{\prime}=\psi_{2}^{\prime}$ in an open set $\mathcal{U} \subset \Sigma$, then $\psi_{1}=\psi_{2}$ in $\mathcal{M} \backslash J^{ \pm}(\Sigma \backslash \operatorname{clos}(\mathcal{U}))$.

This proposition follows from estimates of energy type. Let us review the geometric origin of such estimates. The geometric picture will be essential for global applications.

Let $\psi$ be a solution of

$$
\square_{g} \psi=0
$$

on a Lorentzian manifold $(\mathcal{M}, g)$. Define

$$
T_{\mu \nu}(\psi)=\partial_{\mu} \psi \partial_{\nu} \psi-\frac{1}{2} g_{\mu \nu} \partial^{\alpha} \psi \partial_{\alpha} \psi
$$


We call $T_{\mu \nu}$ the energy-momentum tensor of $\psi^{28}$ Note the symmetry property

$$
T_{\mu \nu}=T_{\nu \mu}
$$

The wave equation (19) implies

$$
\nabla^{\mu} T_{\mu \nu}=0 .
$$

Given a vector field $V^{\mu}$, we may define the associated currents

$$
\begin{aligned}
& J_{\mu}^{V}(\psi)=V^{\nu} T_{\mu \nu}(\psi) \\
& K^{V}={ }^{V} \pi_{\mu \nu} T^{\mu \nu}(\psi)
\end{aligned}
$$

where ${ }^{X} \pi$ is the deformation tensor defined by

$$
{ }^{X} \pi_{\mu \nu}=\frac{1}{2} \nabla_{(\mu} X_{\nu)}=\frac{1}{2}\left(\mathcal{L}_{X} g\right)_{\mu \nu} .
$$

The identity (21) gives

$$
\nabla^{\mu} J_{\mu}^{V}(\psi)=K^{V}(\psi)
$$

In this context, we call $V$ a vector field multiplier.

Note that $J_{\mu}^{V}(\psi)$ and $K^{V}(\psi)$ both depend only on the 1-jet of $\psi$, yet the latter is the divergence of the former. Applying the divergence theorem, this allows one to relate quantities of the same order.

The existence of a tensor $T_{\mu \nu}(\psi)$ satisfying (21) follows from the fact that equation (19) derives from a Lagrangian of a specific type. These issues were first systematically studied by Noether [116]. For more general such Lagrangian theories, two currents $J_{\mu}, K$ with $\nabla^{\mu} J_{\mu}=K$, both depending only on the 1-jet, but not necessarily arising from $T_{\mu \nu}$ as above, are known as compatible currents. These have been introduced and classified by Christodoulou [41].

Higher order estimates arise by commuting the wave equation with other vector fields, and then applying multipliers as above.

Proposition 4.2.2. Let $\psi$ be a solution of the equation of the scalar equation

and $W$ be a vectorfield. Then

$$
\square_{g} \psi=f,
$$

$$
\square_{g}(W \psi)=W(f)-2^{W} \pi^{\alpha \beta} \nabla_{\alpha} \nabla_{\beta} \psi-2\left(2\left(\nabla^{\alpha} W_{\alpha \mu}\right)-\left(\nabla_{\mu}{ }^{W} \pi_{\alpha}^{\alpha}\right)\right) \nabla^{\mu} \psi .
$$

In this context we call $W$ a vector field commutator.

We may obtain thus divergence identities for $J^{V}\left(W_{1} \ldots W_{p} \psi\right)$ where $V$ is an arbitrary multiplier and $W_{i}$ are a string of commutators, relating quantities of the same order. When $V$ is timelike and $\Sigma$ spacelike then at every point

$$
J_{\mu}^{V}(\xi) n_{\Sigma}^{\mu} \sim \sum_{i=0}^{3}\left(\partial_{i} \xi\right)^{2}
$$

\footnotetext{
${ }^{28}$ Note that this is the same expression that appears on the right hand side of (5) in the Einstein-scalar field system. See Section 2.2.2.
} 
where the constants depend on the choice of $V$. This allows one to obtain control of positive definite quantities.

Proposition 4.2.1 can be obtained applying an arbitrary smooth timelike $V$ as a multiplier, and commuting with $W_{i}$ a basis for the tangent space. For global results, careful choice of multipliers and commutators is paramount. The choices will be very related to geometric features of spacetime.

4.2.1. The Kay-Wald boundedness theorem. We begin with the case of Schwarzschild.

We will be interested in understanding the global behaviour of $\psi$ in the exterior of the black hole and white hole regions, up to and including the horizons. It is enough of course to understand the behaviour in the region

$$
\mathcal{D} \doteq \operatorname{clos}\left(J^{-}\left(\mathcal{I}_{A}^{+}\right) \cap J^{+}\left(\mathcal{I}_{A}^{-}\right)\right) \cap \mathcal{Q}
$$

where $\mathcal{I}_{A}^{ \pm}$denote a pair of connected components of $\mathcal{I}^{ \pm}$, respectively, with a common limit point. ${ }^{29}$

Moreover, it suffices to assume that $\Sigma \cap \mathcal{H}^{-}=\varnothing$, and that we are interested in the behaviour in $J^{-}\left(\mathcal{I}^{+}\right) \cap J^{+}(\Sigma)$. Note that in this case, by the domain of dependence property of the above proposition, we have that the solution in this region is determined by $\left.\psi\right|_{\mathcal{D} \cap \Sigma},\left.\psi^{\prime}\right|_{\mathcal{D} \cap \Sigma}$. In the case where $\Sigma$ itself is spherically symmetric, then its projection to $\mathcal{Q}$ will look like:

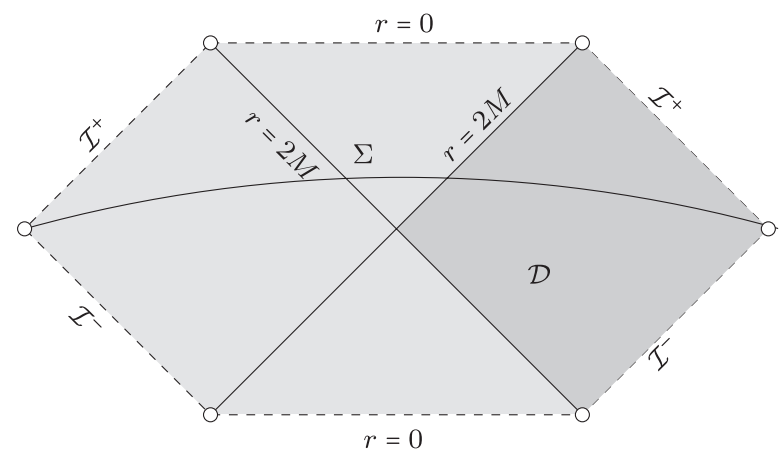

If $\Sigma$ is not itself spherically symmetric, then its projection to $\mathcal{Q}$ will in general have open interior. Nonetheless, we shall always depict $\Sigma$ as above.

The most basic problem is to obtain uniform boundedness for $\psi$. This is resolved in the celebrated:

TheOREM 4.1. Let $(\mathcal{M}, g)$ be Schwarzschild and $\Sigma$ a Cauchy hypersurface, and let $\psi, \psi, \psi^{\prime}$ be as in Proposition 4.2.1 with $\psi \in H_{\mathrm{loc}}^{m+1}(\Sigma)$, $\psi^{\prime} \in H_{\mathrm{loc}}^{m}(\Sigma)$ for a sufficiently high $m$, and such that $\psi, \psi^{\prime}$ decay suitably at $i^{0}$. Then there is a constant $D$ depending on $\psi, \psi^{\prime}$ such that

$$
|\psi| \leq D
$$

in $\mathcal{D}$.

\footnotetext{
${ }^{29}$ We will sometimes be sloppy with distinguishing between $\pi^{-1}(p)$ and $p$, where $\pi: \mathcal{M} \rightarrow \mathcal{Q}$ denotes the natural projection, distinguishing $J^{-}(p)$ and $J^{-}(p) \cap \mathcal{Q}$, etc. The context should make clear what is meant.
} 
The original proof of this theorem is due to Wald [141] and KayWald [89]. The completely standard "easy part" of the proof is a classic application of vector field commutators and multipliers, together with elliptic estimates and the Sobolev inequality. We review this below.

Let $\varphi_{t}$ denote the 1-parameter group of diffeomorphisms generated by the Killing field $T=\partial_{t}$. Define $\Sigma_{\tau}=\varphi_{t}(\Sigma \cap \mathcal{D})$. We have that $\left\{\Sigma_{\tau}\right\}_{\tau \geq 0}$ defines a spacelike foliation of

$$
\mathcal{R} \doteq \cup_{\tau \geq 0} \Sigma_{\tau}
$$

Define

$$
\mathcal{H}^{+}(0, \tau) \doteq \mathcal{H}^{+} \cap J^{+}\left(\Sigma_{0}\right) \cap J^{-}\left(\Sigma_{\tau}\right),
$$

and

$$
\mathcal{R}(0, \tau) \doteq \cup_{0 \leq \bar{\tau} \leq \tau} \Sigma_{\bar{\tau}} .
$$

Let $n_{\Sigma}^{\mu}$ denote the future directed unit normal of $\Sigma$, and let $n_{\mathcal{H}}^{\mu}$ define a null generator of $\mathcal{H}^{+}$, and give $\mathcal{H}^{+}$the associated volume form.

Let $J_{\mu}^{T}(\psi)$ denote the energy current defined by applying the vector field $T$ as a multiplier, i.e.

$$
J_{\mu}^{T}(\psi)=T_{\mu \nu}(\psi) T^{\nu}=\left(\partial_{\mu} \psi \partial_{\nu} \psi-\frac{1}{2} g_{\mu \nu} \partial^{\alpha} \psi \partial_{\alpha} \psi\right) T^{\nu}
$$

with its associated current $K^{T}(\psi)$,

$$
K^{T}(\psi)={ }^{T} \pi^{\mu \nu} T_{\mu \nu}(\psi)=\nabla^{\mu} J_{\mu}^{T}(\psi) .
$$

Since $T$ is Killing, and $\nabla^{\mu} T_{\mu \nu}=0$, it follows that $K^{T}(\psi)=0$, and the divergence theorem applied to $J_{\mu}^{T}$ in the region $\mathcal{R}(0, \tau)$ yields

$$
\int_{\Sigma_{\tau}} J_{\mu}^{T}(\psi) n_{\Sigma_{\tau}}^{\mu}+\int_{\mathcal{H}^{+}(0, \tau)} J_{\mu}^{T}(\psi) n_{\mathcal{H}}^{\mu}=\int_{\Sigma_{0}} J_{\mu}^{T}(\psi) n_{\Sigma_{0}}^{\mu} .
$$

See

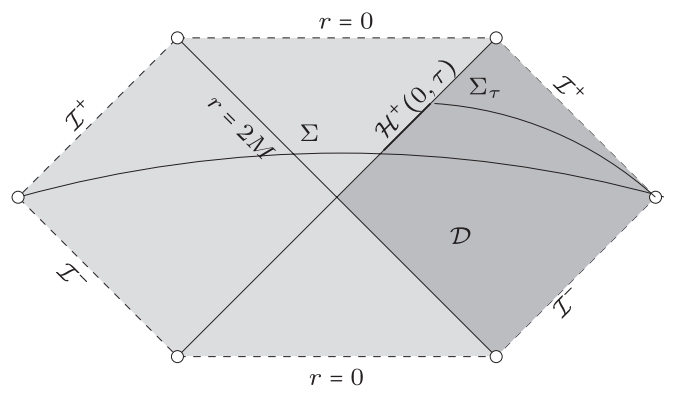

Since $T$ is future-directed causal in $\mathcal{D}$, we have

$$
J_{\mu}^{T}(\psi) n_{\Sigma}^{\mu} \geq 0, \quad J_{\mu}^{T}(\psi) n_{\mathcal{H}}^{\mu} \geq 0 .
$$

Let us fix an $r_{0}>2 M$. It follows from (24), (25) that

$$
\int_{\Sigma_{\tau} \cap\left\{r \geq r_{0}\right\}} J_{\mu}^{T}(\psi) n_{\Sigma_{\tau}}^{\mu} \leq \int_{\Sigma_{0}} J_{\mu}^{T}(\psi) n_{\Sigma_{0}}^{\mu} .
$$


As long as $-g\left(T, n_{\Sigma_{0}}\right) \leq B$ for some constant $B$, we have in fact

$$
\begin{aligned}
& B\left(r_{0}, \Sigma\right)\left(\left(\partial_{t} \psi\right)^{2}+\left(\partial_{r} \psi\right)^{2}+|\not \nabla \psi|^{2}\right) \geq J_{\mu}^{T}(\psi) n^{\mu} \geq b\left(r_{0}, \Sigma\right)\left(\left(\partial_{t} \psi\right)^{2}\right. \\
& \left.\quad+\left(\partial_{r} \psi\right)^{2}+|\not \nabla \psi|^{2}\right) .
\end{aligned}
$$

Here, $|\not \nabla \psi|^{2}$ denotes the induced norm on the group orbits of the $\mathrm{SO}(3)$ action, with $\nabla$ the gradient of the induced metric on the group orbits. We thus have

$$
\int_{\Sigma_{\tau} \cap\left\{r \geq r_{0}\right\}}\left(\partial_{t} \psi\right)^{2}+\left(\partial_{r} \psi\right)^{2}+|\not \nabla \psi|^{2} \leq B\left(r_{0}, \Sigma\right) \int_{\Sigma_{0}} J_{\mu}^{T}(\psi) n_{\Sigma_{0}}^{\mu}
$$

We may now commute the equation with $T$ : Since $[\square, T]=0$, if $\square_{g} \psi=0$ then $\square_{g}(T \psi)=0$. We thus obtain an estimate

$$
\int_{\Sigma_{\tau} \cap\left\{r \geq r_{0}\right\}}\left(\partial_{t}^{2} \psi\right)^{2}+\left(\partial_{r} \partial_{t} \psi\right)^{2}+\left|\not \nabla \partial_{t} \psi\right|^{2} \leq B\left(r_{0}, \Sigma\right) \int_{\Sigma_{0}} J_{\mu}^{T}(T \psi) n_{\Sigma_{0}}^{\mu}
$$

By elliptic estimates and a Sobolev estimate, it follows that if $\psi(x) \rightarrow 0$ as $x \rightarrow i_{0}$, then (27) implies that for $r \geq r_{0}$,

$$
|\psi|^{2} \leq B\left(r_{0}, \Sigma\right)\left(\int_{\Sigma_{0}} J_{\mu}^{T}(\psi) n_{\Sigma_{0}}^{\mu}+\int_{\Sigma_{0}} J_{\mu}^{T}(T \psi) n_{\Sigma_{0}}^{\mu}\right)
$$

for solutions $\psi$ of $\square_{g} \psi=0$.

This concludes the "easy part" of Theorem 4.1. Unfortunately, the constant $B\left(r_{0}, \Sigma\right) \rightarrow \infty$ as $r_{0} \rightarrow 2 M$. Nonetheless, Kay and Wald were able to obtain boundedness all the way up to $\mathcal{H}^{+}$by a number of clever arguments: The degeneration of (28) at the event horizon was overcome by applying the estimate to an auxiliary $\hat{\psi}$ constructed from $\psi$ by inverting an elliptic operator associated to the so-called optical metric, and commuting also with a basis $\Omega_{i}$ for the $s o(3)$ Lie algebra. This inversion required however that $\psi$ vanish at $\mathcal{H}^{+} \cap \mathcal{H}^{-}$. This unphysical assumption was then overcome by appealing to a discrete symmetry of maximal Schwarzschild, interchanging the two ends. See [63] for details.

Unfortunately, the arguments above rely on so much of the special structure of the Schwarzschild solution, that they are not at all robust to perturbation. Moreover, the Kay and Wald proof cannot obtain estimates for higher transversal derivatives to the horizon. To eventually go beyond Schwarzschild, it is clear that one must first understand Theorem 4.1 in a more robust way.

4.3. The red-shift and a new proof of boundedness. We give in this section a new proof of boundedness which overcomes the shortcomings outlined above. In essence, the previous proof limited itself by relying solely on Killing fields as multipliers and commutators. It turns out that there is an important physical aspect of Schwarzschild which can be captured by other vector-field multipliers and commutators which are not however Killing. This is related to the celebrated red-shift effect. 
4.3.1. The classical red-shift. The red-shift effect is one of the most celebrated aspects of black holes. It is classically described as follows: Suppose two observers, $A$ and $B$ are such that $A$ crosses the event horizon and $B$ does not. If $A$ emits a signal at constant frequency as he measures it, then the frequency at which it is received by $B$ is "shifted to the red".

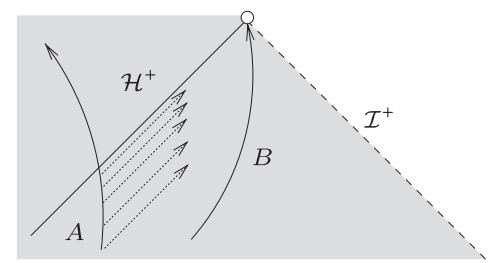

The consequences of this for the appearance of a collapsing star to faraway observers were first explored in the seminal paper of OppenheimerSnyder [117]. For a nice discussion, see also the classic textbook [109].

The red-shift effect as described above is a global one, and essentially depends only on the fact that the proper time of $B$ is infinite whereas the proper time of $A$ before crossing $\mathcal{H}^{+}$is finite. In the case of the Schwarzschild black hole, there is a "local" version of this red-shift: If $B$ also crosses the event horizon but at advanced time later than $A$ :

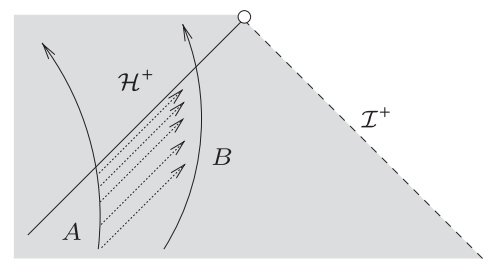

then the frequency at which $B$ receives at his horizon crossing time is shifted to the red by a factor depending exponentially on the advanced time difference of the crossing points of $A$ and $B$.

The exponential factor is determined by the so-called surface gravity, a quantity that can in fact be defined for all so-called Killing horizons. This localised red-shift effect depends only on the positivity of this quantity. Let us explore how we can analytically "capture" this red-shift effect in the Schwarzschild geometry.

4.3.2. The redshift effect as a multiplier and commutator estimate. It turns out that a "vector field multiplier" version of this localised red-shift effect is captured by the following

Proposition 4.3.1. There exists a $\varphi_{t}$-invariant smooth future-directed timelike vector field $N$ on $\mathcal{R}$ and a positive constant $b>0$ such that

$$
K^{N}(\psi) \geq b J_{\mu}^{N}(\psi) N^{\mu}
$$

on $\mathcal{H}^{+}$for all solutions $\psi$ of $\square_{g} \psi=0$.

We can moreover clearly choose this $N$ so that $N=T$ far from $\mathcal{H}^{+}$. 
The localised red-shift effect also has a "vector field commutator" manifestation:

THEOREM 4.2. Under the assumptions of the above theorem, let $Y=$ $N-T$. If $\psi$ satisfies $\square_{g} \psi=0$, then for all $k \geq 1$.

$$
\square_{g}\left(Y^{k} \psi\right)=b_{k+1} Y^{k+1} \psi+\sum_{0 \leq|m| \leq k+1,0 \leq m_{4}<k+1} c_{m} E_{1}^{m_{1}} E_{2}^{m_{2}} T^{m_{3}} Y^{m_{4}} \psi
$$

on $\mathcal{H}^{+}$, where $b_{k}>0$.

The positivity of $b$ and $b_{k}$ is related to the surface gravity. In fact, analogues of the above propositions hold for general stationary spacetimes with a Killing horizon with positive surface gravity.

It turns out that one can interpolate the estimate (26) of Section 4.2.1 with the estimate for $J^{N}$ arising from Proposition 4.3.1. Essential use is made of the positive definite spacetime term $K^{N}(\psi)$ near $\mathcal{H}^{+}$. This yields an estimate for

$$
\int_{\Sigma(\tau)} J_{\mu}^{N}(\psi) n_{\Sigma}^{\mu}
$$

Note that, in contrast with $\int_{\Sigma(\tau)} J_{\mu}^{T}(\psi) n_{\Sigma}^{\mu}$, the integrand of (30) does not degenerate on $\mathcal{H}^{+}$. Higher order estimates can be obtained by commuting with $T$ and in addition with $Y$ above. The most dangerous error term on the right hand side of (29) is the first, and the positivity of $b_{k}$ ensures that it arises with a favourable sign.

4.3.3. A robust boundedness statement. We obtain finally (see [63])

THEOREM 4.3. Let $\Sigma$ be a Cauchy hypersurface for Schwarzschild such that $\Sigma \cap \mathcal{H}^{-}=\varnothing$, let $\Sigma_{0}=\mathcal{D} \cap \Sigma$, let $\Sigma_{\tau}$ denote the translation of $\Sigma_{0}$, let $n_{\Sigma_{\tau}}$ denote the future normal of $\Sigma_{\tau}$, and let $\mathcal{R}=\cup_{\tau \geq 0} \Sigma_{\tau}$. Assume $-g\left(n_{\Sigma_{0}}, T\right)$ is uniformly bounded. Then there exists a constant $C$ depending only on $\Sigma_{0}$ such that the following holds. Let $\psi, \psi, \psi^{\prime}$ be as in Proposition 4.2.1, with $\psi \in H_{\mathrm{loc}}^{k+1}(\Sigma), \psi^{\prime} \in H_{\mathrm{loc}}^{k}(\Sigma)$, and

$$
\int_{\Sigma_{0}} J_{\mu}^{T}\left(T^{m} \psi\right) n_{\Sigma_{0}}^{\mu}<\infty
$$

for $0 \leq m \leq k$. Then

$$
\left|\nabla^{\Sigma_{\tau}} \psi\right|_{H^{k}\left(\Sigma_{\tau}\right)}+|n \psi|_{H^{k}\left(\Sigma_{\tau}\right)} \leq C\left(\left|\nabla^{\Sigma_{0}} \psi\right|_{H^{k}\left(\Sigma_{0}\right)}+\left|\psi^{\prime}\right|_{H^{k}\left(\Sigma_{0}\right)}\right) .
$$

If $k \geq 1$, then we have

$$
\begin{aligned}
& \sum_{0 \leq m \leq k-1} \sum_{m_{1}+m_{2}=m, m_{i} \geq 0}\left|\left(\nabla^{\Sigma}\right)^{m_{1}} n^{m_{2}} \psi\right| \\
& \leq C\left(\lim _{x \rightarrow i^{0}}|\psi|+\left|\nabla^{\Sigma(0)} \psi\right|_{H^{k}\left(\Sigma_{0}\right)}+\left|\psi^{\prime}\right|_{H^{k}\left(\Sigma_{0}\right)}\right)
\end{aligned}
$$

in $\mathcal{R}$. 
Note that $\left(\nabla^{\Sigma}\right)^{m_{1}} n^{m_{2}} \psi$ denotes an $m_{1}$-tensor on the Riemannian manifold $\Sigma_{\tau}$, and $|\cdot|$ on the left hand side of the last inequality above just denotes the induced norm on such tensors.

4.3.4. Perturbing? The validity of Proposition 4.3.1 rests only on the fact that the horizon $\mathcal{H}^{+}$is Killing with positive surface gravity $\kappa>0$.

THEOREM 4.4. Let $g$ be another asymptotically flat metric on $\mathcal{R}$ such that $\mathcal{H}^{+}$is a Killing horizon with positive surface gravity and $\partial_{t}$ is Killing and causal. Then the statement of Theorem 4.3 holds.

In particular, it holds for instance for the Reissner-Nordström metrics in the entire nonextremal range $0 \leq|Q|<M$. The point of the above theorem, however, is that the spherical symmetry plays no role in its proof.

Unfortunately, the above proof cannot apply in Kerr, not on account of its lack of spherical symmetry, but on account of the fact that the Killing field $\partial_{t}$ becomes spacelike in $\mathcal{R}$. This discussion may suggest that one should not expect an argument proving only boundedness for Kerr, that is to say, a "stable argument" would of necessity need to prove more than boundedness, i.e. decay. We shall see later that there is a sense in which this is true and a sense in which it is not! But before exploring this, let us understand how one can go beyond boundedness and prove quantitative decay for waves on Schwarzschild itself. It is quantitative decay after all that we must understand if we are to understand nonlinear problems.

4.4. Quantitative decay for the wave equation on Schwarzschild. Quantitative decay rates are central for our understanding of nonlinear problems. To discuss energy decay for solutions $\psi$ of $\square_{g} \psi=0$ on Schwarzschild, one must consider a different foliation. Let $\tilde{\Sigma}_{0}$ be a spacelike hypersurface terminating on null infinity and define $\tilde{\Sigma}_{\tau}$ by translation.

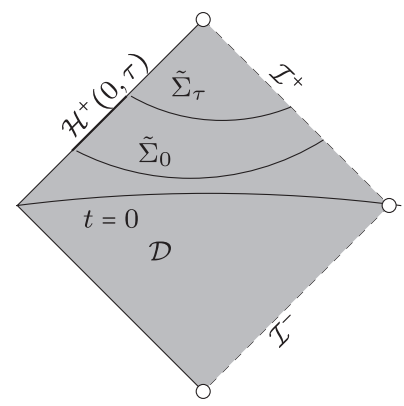

The main result of this section is the following

THEOREM 4.5. There exists a constant $C$ depending only on $\tilde{\Sigma}_{0}$ such that the following holds. Let $\psi \in H_{\mathrm{loc}}^{4}, \psi^{\prime} \in H_{\mathrm{loc}}^{3}$, and suppose $\lim _{x \rightarrow i^{0}} \psi=0$ and

$$
E_{1}=\sum_{|(\alpha)| \leq 3} \sum_{\Gamma=\left\{\Omega_{i}\right\}} \int_{t=0} r^{2} J_{\mu}^{n_{0}}\left(\Gamma^{(\alpha)} \psi\right) n_{0}^{\mu}<\infty
$$


where $n_{0}$ denotes the unit normal of the hypersurface $\{t=0\}$. Then

$$
\int_{\tilde{\Sigma}_{\tau}} J_{\mu}^{N}(\psi) n_{\tilde{\Sigma}_{\tau}}^{\mu} \leq C E_{1} \tau^{-2}
$$

where $N$ is the vector field of Section 4.3.2. Now let $\psi \in H_{\mathrm{loc}}^{7}, \psi^{\prime} \in H_{\mathrm{loc}}^{6}$, $\lim _{x \rightarrow i^{0}} \psi=0$, and suppose

$$
E_{2}=\sum_{|(\alpha)| \leq 6} \sum_{\Gamma=\left\{\Omega_{i}\right\}} \int_{t=0} r^{2} J_{\mu}^{n_{0}}\left(\Gamma^{(\alpha)} \psi\right) n_{0}^{\mu}<\infty .
$$

Then

$$
\sup _{\tilde{\Sigma}_{\tau}}|\psi| \leq C \sqrt{E_{2}} \tau^{-1}, \quad \sup _{\tilde{\Sigma}_{\tau}}|r \psi| \leq C \sqrt{E_{2}} \tau^{-1 / 2} .
$$

The fact that (31) "loses derivatives" is a fundamental aspect of this problem related to the trapping phenomenon, to be discussed in what follows, although the precise number of derivatives lost above is wasteful.

We can also express the pointwise decay in terms of advanced and retarded null coordinates $u$ and $v$. Defining $v=\left(t+r^{*}\right)=(t+r+2 M \log (r-$ $2 M)), u=\left(t-r^{*}\right)=(t-r-2 M \log (r-2 M))$, we have

$$
|\psi| \leq C E_{2}(|v|+1)^{-1}, \quad|r \psi| \leq C\left(r_{0}\right) E_{2} u^{-\frac{1}{2}},
$$

where the first inequality applies in $\mathcal{D} \cap \operatorname{clos}(\{t \geq 0\})$, whereas the second applies only in $\mathcal{D} \cap\{t \geq 0\} \cap\left\{r \geq r_{0}\right\}$, with $C\left(r_{0}\right) \rightarrow \infty$ as $r_{0} \rightarrow 2 M$. Note that, as in Minkowski space, the first inequality of (33) is sharp as a uniform decay rate in $v$.

4.4.1. The $X$-multiplier and integral decay. The zero'th step in the proof of Theorem 4.5 is an estimate for a spacetime integral whose integrand should control the quantity

$$
\chi J_{\mu}^{N}(\psi) N^{\mu}
$$

where $\chi$ is a $\varphi_{t}$-invariant weight function such that $\chi$ degenerates only at infinity and $N$ is as in Section 4.3.2. Estimates of the spacetime integral (34) have their origin in the classical virial theorem, which in Minkowski space essentially arises from applying the energy identity to the current $J^{V}$ with $V=\frac{\partial}{\partial r}$.

Naively, one might expect to be able to obtain an estimate of the form say

$$
\int_{\tilde{\mathcal{R}}(0, \tau)} \chi J_{\mu}^{N}(\psi) N^{\mu} \leq B \int_{\tilde{\Sigma}_{0}} J_{\mu}^{N} n_{\tilde{\Sigma}_{0}}^{\mu}
$$

for such a $\chi$. It turns out that there is a well known high-frequency obstruction for the existence of an estimate of the form (35) arising from trapped null geodesics. This problem has been long studied in the context of the wave equation in Minkowski space outside of an obstacle, where the analogue of trapped null geodesics are straight lines which reflect off the obstacle's boundary in such a way so as to remain in a compact subset of space. In 
Schwarzschild, one can easily infer from a continuity argument the existence of a family of null geodesics with $i^{+}$as a limit point. ${ }^{30}$ But in view of the integrability of geodesic flow, one can in fact understand all such geodesics explicitly.

It turns out that the hypersurface $r=3 M$ is spanned by null geodesics. This timelike hypersurface $r=3 M$ is traditionally called the photon sphere. From every point in the $\mathcal{R}$, there is a codimension-one subset of future directed null directions whose corresponding geodesics approach $r=3 M$, and all other null geodesics either cross $\mathcal{H}^{+}$or meet $\mathcal{I}^{+}$.

We may capture the trapping by constructing a current $J^{X}$ whose associated current $K^{X}$ is positive semidefinite in highest derivatives, degenerating precisely at the photon sphere.

The construction is delicate. See [61]. A suitable current turns out to be:

$$
\begin{aligned}
J_{\mu}^{\mathbf{X}}(\psi)= & e J_{\mu}^{N}(\psi)+J_{\mu}^{X^{a}}(\psi)+\sum_{i} J_{\mu}^{X^{b}, w^{b}}\left(\Omega_{i} \psi\right) \\
& -\frac{1}{2} \frac{r\left(f^{b}\right)^{\prime}}{f^{b}(r-2 M)}\left(\frac{r-2 M}{r^{2}}-\frac{\left(r^{*}-\alpha-\alpha^{1 / 2}\right)}{\alpha^{2}+\left(r^{*}-\alpha-\alpha^{1 / 2}\right)^{2}}\right) X_{\mu}^{b} \psi^{2} .
\end{aligned}
$$

Here, $N$ is as in Section 4.3.2, $r^{*}=r+2 M \log (r-2 M)-3 M-2 M \log M$, $X^{a}=f^{a} \partial_{r^{*}}, X^{b}=f^{b} \partial_{r^{*}}$,

$$
\begin{gathered}
f^{a}=-\frac{C_{a}}{\alpha r^{2}}+\frac{c_{a}}{r^{3}} \\
f^{b}=\frac{1}{\alpha}\left(\tan ^{-1} \frac{r^{*}-\alpha-\alpha^{1 / 2}}{\alpha}-\tan ^{-1}\left(-1-\alpha^{-1 / 2}\right)\right), \\
w^{b}=\frac{1}{8}\left(\left(f^{b}\right)^{\prime}+2 \frac{r-2 M}{r^{2}} f^{b}\right),
\end{gathered}
$$

and for general $X, w$, the $J^{X, w}, K^{X, w}$ are the "modified" currents

$$
\begin{gathered}
J_{\mu}^{X, w}=J_{\mu}^{X}(\psi)+\frac{1}{8} w \partial_{\mu}\left(\psi^{2}\right)-\frac{1}{8}\left(\partial_{\mu} w\right) \psi^{2} \\
K^{X, w}=\nabla^{\mu} J_{\mu}^{X, w},
\end{gathered}
$$

and $e, C_{a}, c_{a}, \alpha$ are positive parameters which must be chosen accordingly.

With these choices, one can show (after some computation) that the divergence $K^{\mathbf{X}}=\nabla^{\mu} J_{\mu}^{\mathbf{X}}$ controls in particular

$$
\int_{\mathbb{S}^{2}} K^{\mathbf{X}}(\psi) \geq b \chi \int_{\mathbb{S}^{2}} J_{\mu}^{N}(\psi) n^{\mu}
$$

where $\chi$ is non-vanishing but decays (polynomially) as $r \rightarrow \infty$. Note that in view of the normalisation of the $r^{*}$ coordinate, $X^{b}=0$ precisely at $r=3 M$.

\footnotetext{
${ }^{30}$ This can be thought of as a very weak notion of what it would mean for a null geodesic to be trapped from the point of view of decay results with respect to the foliation $\tilde{\Sigma}_{\tau}$.
} 
The quantity $K^{\mathbf{X}}$ controls in fact also second order derivatives of $\psi$ but this control degenerates precisely at $r=3 M$.

On the other hand, we can control the spacelike boundary terms by the the energy we have shown to be bounded

$$
\left|J_{\mu}^{\mathbf{X}}(\psi) n_{\tilde{\Sigma}_{\tau}}^{\mu}\right| \leq B\left(J_{\mu}^{N}(\psi) n_{\tilde{\Sigma}_{\tau}}^{\mu}+\sum_{i=1}^{3} J_{\mu}^{N}\left(\Omega_{i} \psi\right) n_{\tilde{\Sigma}_{\tau}}^{\mu}\right)
$$

and the boundary term on the event horizon $\mathcal{H}^{+}$by

$$
-J_{\mu}^{\mathbf{X}}(\psi) n_{\mathcal{H}^{+}}^{\mu} \leq B\left(J_{\mu}^{T}(\psi) n_{\mathcal{H}^{+}}^{\mu}+\sum_{i=1}^{3} J_{\mu}^{T}\left(\Omega_{i} \psi\right) n_{\mathcal{H}^{+}}^{\mu}\right) .
$$

Applying the divergence theorem in $\mathcal{R}\left(\tau^{\prime}, \tau\right)$ and the previous Theorem 4.3, one obtains the integrated decay estimate:

$$
\int_{\tilde{\mathcal{R}}\left(\tau^{\prime}, \tau\right)} \chi J_{\nu}^{N}(\psi) N^{\mu} \leq B \int_{\tilde{\Sigma}\left(\tau^{\prime}\right)}\left(J_{\mu}^{N}(\psi)+\sum_{i=1}^{3} J_{\mu}^{N}\left(\Omega_{i} \psi\right)\right) n_{\tilde{\Sigma}_{\tau^{\prime}}}^{\mu} .
$$

4.4.2. The $Z$-multiplier. How does the estimate (39) assist us to prove decay?

Recall that energy decay can be proven in Minkowski space with the help of the so-called Morawetz current. Let

$$
Z=u^{2} \partial_{u}+v^{2} \partial_{v}
$$

and define

$$
J_{\mu}^{Z, w}(\psi)=J_{\mu}^{Z}(\psi)+\frac{t r^{*}(1-2 M / r)}{2 r} \psi \partial_{\mu} \psi-\frac{r^{*}(1-2 M / r)}{4 r} \psi^{2} \partial_{\mu} t .
$$

Setting $M=0$, this corresponds precisely to the current introduced by Morawetz [111] to prove decay for scalar waves outside a convex obstacle on Minkowski space.

It is interesting to note that this current is $C^{0}$ but not $C^{1}$ across $\mathcal{H}^{+} \cup \mathcal{H}^{-}$.

To understand how one hopes to use this current, let us recall the situation in Minkowski space. There, the signifance of (40) arises since it is a conformal Killing field. Setting $M=0, r^{*}=r$ in the above one obtains ${ }^{31}$

$$
\begin{gathered}
\int_{t=\tau} J_{\mu}^{Z, w} n^{\mu} \geq 0, \\
K^{Z, w}=0 .
\end{gathered}
$$

\footnotetext{
${ }^{31}$ The reason for introducing the 0 'th order terms is because the wave equation is not conformally invariant. It is remarkable that one can nonetheless obtain positive definite boundary terms, although a slightly unsettling feature is that this positivity property (41) requires looking specifically at constant $t=\tau$ surfaces and integrating.
} 
The inequality (41) remains true in the Schwarzschild case and one can obtain exactly as before

$$
\int_{t=\tau} J_{\mu}^{Z, w} n^{\mu} \geq b \int_{t=\tau} u^{2}\left(\partial_{u} \psi\right)^{2}+v^{2}\left(\partial_{v} \psi\right)^{2}+\left(1-\frac{2 M}{r}\right)\left(u^{2}+v^{2}\right)|\not \nabla \psi|^{2} .
$$

(In fact, we have dropped positive 0'th order terms from the right hand side of (43), which will be useful for us later on in Section 4.4.3.) Note that away from the horizon, we have that

$$
\int_{t=\tau} J_{\mu}^{Z, w} n^{\mu} \geq b\left(r_{0}, R\right) \tau^{2} \int_{\{t=\tau\} \cap\left\{r_{0} \leq r \leq R\right\}} J_{\mu}^{N} n^{\mu} .
$$

Thus, if the left hand side of (44) could be shown to be bounded, this would prove the first statement of Theorem 4.5 where $\tilde{\Sigma}_{\tau}$ is replaced however with $\{t=\tau\} \cap\left\{r_{0} \leq r \leq R\right\}$.

In the case of Minkowski space, the boundedness of the left hand side of (43) follows immediately by (42) and the energy identity

$$
\int_{t=\tau} J_{\mu}^{Z, w}+\int_{0 \leq t \leq \tau} K^{Z, w}=\int_{t=0} J_{\mu}^{Z, w}
$$

as long as the data are suitably regular and decay so as for the right hand side above to be bounded. For Schwarzschild, one cannot expect (42) to hold, and this is why we have introduced the $X$-related currents.

First the good news: There exist constants $r_{0}<R$ such that

$$
K^{Z, w} \geq 0
$$

for $r \leq r_{0}$, and in fact

$$
K^{Z, w} \geq b \frac{t}{r^{3}} \psi^{2}
$$

for $r \geq R$ and some constant $b$. These terms have the "right sign" in the energy identity (45). In $\left\{r_{0} \leq r \leq R\right\}$, however, the best we can do is

$$
-K^{Z, w} \leq B t\left(|\not \nabla|^{2}+|\psi|^{2}\right) .
$$

Remarkable, the above inequality together with (39) is sufficient to obtain the energy decay statement (31) by a dyadic iteration argument. See $[\mathbf{5 9}, \mathbf{6 3}]$ for details.

4.4.3. Pointwise estimates. To derive pointwise decay for $\psi$ itself, we should remember that we have in fact dropped a good 0'th order term from the estimate (43). In particular, we have also

$$
\int_{t=\tau} J_{\mu}^{Z, w}(\psi) n^{\mu} \geq b \int_{\{t=\tau\} \cap\left\{r \geq r_{0}\right\}}\left(\tau^{2} r^{-2}+1\right) \psi^{2} .
$$

From this and the previously derived bounds, pointwise decay can be shown easily by applying $\Omega_{i}$ as commutators and Sobolev estimates. See [59] for details. 
4.4.4. Comments and further reading. The origin of the use of vector field multipliers of the type $X$ and $Z$ for proving decay for solutions of the wave equation goes back to Morawetz. The first results in the direction of the estimate (39) were in the pioneering work of Laba and Soffer [101] and Blue and Soffer $[\mathbf{1 6}]$. See also $[\mathbf{1}, \mathbf{1 7}, \mathbf{1 8}, \mathbf{1 0 6}]$.

The decay result Theorem 4.5 was obtained in our [59]. A result yielding similar decay away from the horizon (but weaker decay along the horizon) was proven independently in a nice paper of Blue and Sterbenz [19]. Statement (32) of Theorem 4.5 has been generalised to the Maxwell case by Blue $[\mathbf{1 5}]$.

The best previously known results on general solutions of the wave equation were non-quantitative decay type statements, scattering, and asymptotic completeness. See $[\mathbf{1 3 9}, \mathbf{6 7}, \mathbf{6 8}, \mathbf{5}, \mathbf{4}, \mathbf{1 1 4}]$. These type of statements are typically insensitive to the amount of trapping. See the related discussion of Section 4.4.6, where the statement of Theorem 4.5 is compared to non-quantitative statements heuristically derived in the physics literature.

4.4.5. Perturbing? Use of the $J^{N}$ current "stabilises" the proof of Theorem 4.5 with respect to considerations near the horizon. There is, however, a sense in which the above argument is still fundamentally attached to Schwarzschild. The approach taken to derive the multiplier estimate (36) depends on the structure of the trapping set, in particular, the fact that trapped null geodesics approach a codimension-1 subset of spacetime, the photon sphere. Overcoming the restrictiveness of this approach is the fundamental remaining difficulty in extending these techniques to Kerr, as will be accomplished in Section 4.6. Precise implications of this fact for multiplier estimates are discussed further in $[\mathbf{1}]$.

4.4.6. Aside: Quantitative vs. non-quantitative results and the heuristic tradition. The study of wave equations on Schwarzschild has a long history in the physics literature, beginning with the pioneering paper of Regge and Wheeler [123]. These studies have all been associated with showing "stability".

A seminal paper is that of Price [122]. There, insightful heuristic arguments were put forth deriving the asymptotic tail of each spherical harmonic $\psi_{\ell}$ evolving from compactly supported initial data, suggesting that for $r>2 M$,

$$
\psi_{\ell}(r, t) \sim C_{\ell} t^{-(3+2 \ell)} .
$$

These arguments were later extended by Gundlach et al. [78] to suggest

$$
\left.\psi_{\ell}\right|_{\mathcal{H}^{+}} \sim C_{\ell} v^{-(3+2 \ell)},\left.\quad r \psi_{\ell}\right|_{\mathcal{I}^{+}} \sim \bar{C}_{\ell} u^{-(2+\ell)} .
$$

Another approach to these heuristics via the analytic continuation of the Green's function was followed by [28]. The latter approach in principle could perhaps be turned into a rigorous proof, at least for solutions not supported on $\mathcal{H}^{+} \cap \mathcal{H}^{-}$. See $[\mathbf{1 0 5}, \mathbf{9 7}]$ for just (47) for the $\ell=0$ case. 
Statements of the form (47) are interesting because, if proven, they would give the fine structure of the tail of the solution. However, it is important to realise that statements like (47) in of themselves would not give quantitative bounds for the size of the solution at all later times in terms of initial data. In fact, the above heuristics do not even suggest what the best such quantitative result would be, they only give a heuristic lower bound on the best possible quantitative decay rate in a theorem like Theorem 4.5.

Let us elaborate on this further. For fixed spherical harmonic, by compactness a statement of the form (47) would immediately yield some bound

$$
\left|\psi_{\ell}\right|(r, t) \leq D\left(r, \psi_{\ell}\right) t^{-3}
$$

for some constant $D$ depending on $r$ and on the solution itself. It is not clear, however, what the sharp such quantitative inequality of the form (49) is supposed to be when the constant is to depend on a natural quantity associated to data. It is the latter, however, which is important for the nonlinear stability problem.

There is a setting in which a quantitative version of (49) has indeed been obtained: The results of [57] (which apply to the nonlinear problem where the scalar field is coupled to the Einstein equation, but which can be specialised to the decoupled case of the $\ell=0$ harmonic on Schwarzschild) prove in particular that

$$
\left|n_{\Sigma_{\tau}} \psi_{0}\right|+\left|\psi_{0}\right| \leq C_{\epsilon} D\left(\psi, \psi^{\prime}\right) \tau^{-3+\epsilon}, \quad\left|r \psi_{0}\right| \leq C D\left(\psi, \psi^{\prime}\right) \tau^{-2}
$$

where $C_{\epsilon}$ depends only on $\epsilon$, and $D\left(\psi, \psi^{\prime}\right)$ is a quantity depending only on the initial $J^{T}$ energy and a pointwise weighted $C^{1}$ norm. In view of the relation between $\tau, u$, and $v,(50)$ includes also decay on the horizon and null infinity as in the heuristically derived (48). The fact that the power 3 indeed appears in both in the quantitative (50) and in (49) may be in part accidental. See also [13].

For general solutions, i.e. for the sum over spherical harmonics, the situation is even worse. In fact, a statement like (47) a priori gives no information whatsoever of any sort, even of the non-quantitative kind. It is in principle compatible with $\lim \sup _{t \rightarrow \infty} \psi(r, t)=\infty .^{32}$ It is well known, moreover, that to understand quantitative decay rates for general solutions, one must quantify trapping. This is not, however, captured by the heuristics leading to (47), essentially because for fixed $\ell$, the effects of trapping concern an intermediate time interval not reflected in the tail. It should thus not be surprising that these heuristics do not address the fundamental problem at hand.

Another direction for heuristic work has been the study of so-called quasi-normal modes. These are solutions with time dependence $e^{-i \omega t}$ for $\omega$ with negative imaginary part, and appropriate boundary conditions. These

\footnotetext{
${ }^{32}$ Of course, given the quantitative result of Theorem 4.3 and the statement (47), one could then infer that for each $r>2 M$, then $\lim _{t \rightarrow \infty} \phi(r, t)=0$, without however a rate.
} 
occur as poles of the analytic continuation of the resolvent of an associated elliptic problem, and in the scattering theory literature are typically known as resonances. Quasinormal modes are discussed in the nice survey article of Kokkotas and Schmidt [95]. Rigorous results on the distribution of resonances have been achieved in Bachelot-Motet-Bachelot [7] and Sá BarretoZworski [127]. The asymptotic distribution of the quasi-normal modes as $\ell \rightarrow \infty$ can be thought to reflect trapping. On the other hand, these modes do not reflect the "low-frequency" effects giving rise to tails. Thus, they too tell only part of the story. See, however, the case of Schwarzschild-de Sitter in Section 4.8 .

Finally, we should mention Stewart [134]. This is perhaps the first clear discussion in the physics literature of the relevance of trapping for the Schwarzschild metric and the difference between quantitative and nonquantitative decay rates. It is interesting to compare Section 3 of [134] with what has now been proven: Although the predictions of $[\mathbf{1 3 4}]$ do not quite match the situation in Schwarzschild (they are in particular incompatible with (47)), they apply well to the Schwarzschild-de Sitter case developed in Section 4.8 .

4.5. Boundedness for axisymmetric black holes. We now turn to the problem of perturbing the Schwarzschild metric and proving boundedness and decay for the wave equation on the backgrounds of such perturbed metrics. Let us recall our dilemma: The boundedness argument of Section 4.2 required that $T$ remains causal everwhere in the exterior. In view of the comments of Section 4.3.4, this is clearly unstable. On the other hand, the decay argument of Section 4.4 requires understanding the trapped set and in particular, uses the fact that in Schwarzschild, a certain codimension-1 subset of spacetime-the photon sphere-plays a special role. Again, as discussed in Section 4.4.5, this special structure is unstable.

It turns out that nonetheless, these issues can be addressed and both boundedness (see Theorem 4.6) and decay (see Theorem 4.8) can be proven for the wave equation on suitable perturbations of Schwarzschild including slowly rotating Kerr. As we shall see, the boundedness proof (See Section 4.5) turns out to be more robust and can be applied to a larger class of metrics-but it too requires some insight from the Schwarzschild decay argument! The decay proof (see Section 4.6) will require us to restrict to exactly Kerr spacetimes.

We will derive a rather general boundedness theorem for a class of axisymmetric stationary black hole exteriors near Schwarzschild. The result (Theorem 4.6) will include slowly rotating Kerr solutions with parameters $|a| \ll M$. We have already explained in what sense the Kerr metric is "close" to Schwarzschild in the region $\mathcal{R}$. Let us note that with respect to the coordinates $r_{\mathrm{Schw}}, t^{*}, \phi^{*}, \theta$ in $\mathcal{R}$, then $\partial_{t^{*}}$ and $\partial_{\phi^{*}}$ are Killing for both the Schwarzschild and the Kerr metric. The class of metrics which will concern us here are metrics defined on $\mathcal{R}$ such that the metric functions are 
close to Schwarzschild in a suitable sense, ${ }^{33}$ and $\partial_{t^{*}}, \partial_{\phi^{*}}$ are Killing, where these are defined with respect to the ambient Schwarzschild coordinates.

There is however an additional geometric assumption we shall need, and this is motivated by a geometric property of the Kerr spacetime, to be described immediately below.

4.5.1. A geometric property of the horizon. Let us here remark a geometric property of the Kerr spacetime itself which turns out to be of utmost importance in what follows: Let $V$ denote a null generator of $\mathcal{H}^{+}$. Then

$$
V \in \operatorname{Span}\left\{\partial_{t^{*}}, \partial_{\phi^{*}}\right\} .
$$

There is a deep reason why this is true. For stationary black holes with nondegenerate horizons, a celebrated argument of Hawking retrieves a second Killing field in the direction of the null generator $V$. Thus, if $\partial_{t^{*}}$ and $\partial_{\phi^{*}}$ span the complete set of Killing fields, then $V$ must evidently be in their span.

In fact, choosing $V$ accordingly we have

$$
V=\partial_{t^{*}}+\left(a / 2 M r_{+}\right) \partial_{\phi^{*}}
$$

4.5.2. The boundedness theorem. Besides closeness to Schwarzschild, (51) is the crucial assumption we shall need. Our boundedness theorem is the following:

THEOREM 4.6. Let $g$ be a metric defined on the differentiable manifold $\mathcal{R}$ with stratified boundary $\mathcal{H}^{+} \cup \Sigma_{0}$, and let $\partial_{t^{*}}$ and $\partial_{\phi^{*}}$ be Schwarzschild Killing fields. Assume

(1) $g$ is sufficiently close to Schwarzschild in an appropriate sense

(2) $\partial_{t^{*}}$ and $\partial_{\phi^{*}}$ are Killing with respect to $g$

(3) $\mathcal{H}^{+}$is null with respect to $g$ and $\partial_{t^{*}}$ and $\partial_{\phi^{*}}$ span the null generator of $\mathcal{H}^{+}$.

Then the statement of Theorem 4.3 holds.

See $[\mathbf{6 2}]$ for the precise formulation of the closeness assumption 1.

Corollary 4.1. The result applies to Kerr, and to the more general Kerr-Newman family (solving Einstein-Maxwell), for parameters $|a| \ll M$ (and also $|Q| \ll M$ in the Kerr-Newman case).

Thus, we have quantitative pointwise and energy bounds for $\psi$ and arbitrary derivatives on slowly rotating Kerr and Kerr-Newman exteriors.

For simplicity, the reader can specialise the discussion below to the case of a Kerr metric with $|a| \ll M$.

\footnotetext{
${ }^{33}$ This requires moving to an auxiliary coordinate system. See [62].
} 
4.5.3. The axisymmetric case. From (52), it follows that there is a constant $\omega_{0}>0$, depending only on the parameters $a$ and $M$, such that if

$$
\left|\partial_{t^{*}} \psi\right|^{2} \geq \omega_{0}\left|\partial_{\phi^{*}} \psi\right|^{2}
$$

on $\mathcal{H}^{+}$, then the flux satisfies

$$
J_{\mu}^{T}(\psi) n_{\mathcal{H}^{+}}^{\mu} \geq 0
$$

Note also that, for fixed $M$, we can take

$$
\omega_{0} \rightarrow 0, \quad \text { as } \quad a \rightarrow 0 .
$$

Note that this holds as well in the case of the general assumptions of Theorem 4.6, where the condition $a \rightarrow 0$ in (55) is now replaced by the condition that the closeness parameter of $g$ to Schwarzschild in the sense of condition 1 is suitably small.

There is an immediate application of (53). Let us restrict for the moment to axisymmetric solutions, i.e. to $\psi$ such that $\partial_{\phi} \psi=0$. It follows that (53) trivially holds. As a result, our argument proving boundedness is stable, i.e. Theorem 4.3 holds for axisymmetric solutions of the wave equation on Kerr spacetimes with $|a| \ll M$. In fact, the restriction on $a$ can be be removed:

THEOREM 4.7. The statement of Theorem 4.3 holds for axisymmetric solutions $\psi_{0}$ of for Kerr-Newman the full subextremal range of parameters $M>0,0 \leq|Q|<\sqrt{M^{2}-a^{2}}$.

4.5.4. Superradiant and non-superradiant frequencies. There is a more general setting where we can make use of (53). Let us suppose for the time being that we could take the Fourier transform $\hat{\psi}(\omega)$ of our solution $\psi$ in $t^{*}$ and then expand in azimuthal modes $\psi_{m}$, i.e. modes associated to the Killing vector field $\partial_{\phi^{*}}$.

If we were to restrict $\psi$ to the frequency range

$$
|\omega|^{2} \geq \omega_{0} m^{2},
$$

then (53) and thus (54) holds after integrating along $\mathcal{H}^{+}$. In view of this, frequencies in the range (56) are known as nonsuperradiant frequencies. The frequency range

$$
|\omega|^{2} \leq \omega_{0} m^{2}
$$

determines the so-called superradiant frequencies. In the physics literature, the main difficulty of this problem has traditionally been perceived to "lie" with these frequencies.

Let us pretend for the time being that using the Fourier transform, we could indeed decompose

$$
\psi=\psi_{\sharp}+\psi_{b}
$$

where $\psi_{\sharp}$ is supported in (56), whereas $\psi_{b}$ is supported in (57). 
In view of the discussion immediately above and the comments of Section 4.5.3, it is plausible to expect that one could indeed prove boundedness for $\psi_{\sharp}$ in the manner of the proof of Theorem 4.3. In particular, if one could localise the integrated version of (54) to arbitrary sufficiently large subsegments $\mathcal{H}\left(\tau^{\prime}, \tau^{\prime \prime}\right)$, one could obtain

$$
\int_{\Sigma_{\tau}} J_{\mu}^{n_{\Sigma_{\tau}}}\left(\psi_{\sharp}\right) n_{\Sigma_{\tau}}^{\mu} \leq B \int_{\Sigma_{0}} J_{\mu}^{n_{\Sigma_{0}}}\left(\psi_{\sharp}\right) n_{\Sigma_{0}}^{\mu} .
$$

This would leave $\psi_{b}$. Since this frequency range does not suggest a direct boundedness argument, it is natural to revisit the decay mechanism of Schwarzschild. We have already discussed (see Section 4.4.5) the instability of the decay argument; this instability arose from the structure of the set of trapped null geodesics. At the heuristic level, however, it is easy to see that, if one can take $\omega_{0}$ sufficiently small, then solutions supported in (57) cannot be trapped. In particular, for $|a| \ll M$, superradiant frequencies for $\square_{g} \psi=0$ on Kerr are not trapped. This will be the fundamental observation allowing for the boundedness theorem. Let us see how this statement can be understood from the point of view of energy currents.

We continue here our heuristic point of view, where we assume a decomposition (58) where $\psi_{b}$ is supported in (57). In particular, one has an inequality

$$
\int_{-\infty}^{\infty} \int_{0}^{2 \pi} \omega_{0}^{2}\left(\partial_{\phi} \psi_{b}\right)^{2} d \phi^{*} d t^{*} \geq \int_{-\infty}^{\infty} \int_{0}^{2 \pi}\left(\partial_{t} \psi_{b}\right)^{2} d \phi^{*} d t^{*}
$$

for all $(r, \theta)$. We shall see below that (60) allows us easily to construct a suitable stable current for Schwarzschild. The choice is actually quite flexible in comparison with the considerations of Section 4.4.1. Our choice (see [62]) is defined by

$$
J^{\mathbf{X}}=e J^{N}+J^{X_{a}}+J^{X_{b}, w_{b}}
$$

where here, $N$ is the vector field of Section 4.3.2, $X_{a}=f_{a} \partial_{r^{*}}$, with

$$
\begin{array}{ll}
f_{a}=-r^{-4}\left(r_{0}\right)^{4}, & \text { for } r \leq r_{0} \\
f_{a}=-1, & \text { for } r_{0} \leq r \leq R_{1}, \\
f_{a}=-1+\int_{R_{1}}^{r} \frac{d \tilde{r}}{4 \tilde{r}}, & \text { for } R_{1} \leq r \leq R_{2}, \\
f_{a}=0, & \text { for } r \geq R_{2},
\end{array}
$$

$X_{b}=f_{b} \partial_{r^{*}}$ with

$$
f_{b}=\chi\left(r^{*}\right) \pi^{-1} \int_{0}^{r^{*}} \frac{\alpha}{x^{2}+\alpha^{2}}
$$


and $\chi\left(r^{*}\right)$ is a smooth cutoff with $\chi=0$ for $r^{*} \leq 0$ and $\chi=1$ for $r^{*} \geq 1$. Here $r$ and $r^{*}$ are Schwarzschild coordinates. ${ }^{34}$ The function $w_{b}$ is given by

$$
w_{b}=f_{b}^{\prime}+\frac{2}{r}(1-2 M / r)(1-M / r) f_{b} .
$$

The parameters $e, \alpha, r_{0}, R_{1}, R_{2}$ must be chosen accordingly!

Restricting to the range (57), using (60), with some computation we would obtain

$$
\int_{-\infty}^{\infty} \int_{0}^{2 \pi} K^{\mathbf{X}}\left(\psi_{b}\right) d \phi^{*} d t^{*} \geq b \int_{-\infty}^{\infty} \int_{0}^{2 \pi} \chi J_{\mu}^{n_{\Sigma}}\left(\psi_{b}\right) n_{\Sigma}^{\mu} d \phi^{*} d t^{*}
$$

for all $(r, \theta)$.

The above inequality can immediately be seen to be stable to metrics in the class allowed in the statement of Theorem 4.6. That is to say, for such metrics, if $\psi_{b}$ is supported in (57) (where frequencies here are defined by Fourier transform in coordinates $\left.t^{*}, \phi^{*}\right)$, then the inequality (62) holds as before. In particular, (62) holds for Kerr for small $|a| \ll M$.

How would (62) give boundedness for $\psi_{b}$ ? We need in fact to suppose something slightly stronger, namely that (62) holds localised to $\mathcal{R}(0, \tau)$. Consider the currents

$$
J=J^{T}+e_{2} J^{\mathbf{X}}, \quad K=\nabla^{\mu} J_{\mu},
$$

where $e_{2}$ is a positive parameters, and $J^{N}$ is the current of Section 4.3.2. Then, for metrics $g$ close enough to Schwarzschild, and for $e_{2}$ sufficiently small, we would have from a localised (62) that

$$
\begin{gathered}
\int_{\mathcal{R}(0, \tau)} K\left(\psi_{b}\right) \geq 0, \\
\int_{\mathcal{H}(0, \tau)} J_{\mu}\left(\psi_{b}\right) n_{\mathcal{H}}^{\mu} \geq 0,
\end{gathered}
$$

and thus

$$
\int_{\Sigma_{\tau}} J_{\mu}\left(\psi_{b}\right) n_{\Sigma_{\tau}}^{\mu} \leq \int_{\Sigma_{0}} J_{\mu}\left(\psi_{b}\right) n_{\Sigma_{0}}^{\mu}
$$

Moreover, for $g$ sufficiently close to Schwarzschild and $e_{1}, e_{2}$ suitably defined, we also have

$$
\int_{\Sigma_{\tau}} J_{\mu}^{n_{\Sigma_{\tau}}}\left(\psi_{b}\right) n^{\mu} \leq B \int_{\Sigma_{\tau}} J_{\mu}\left(\psi_{b}\right) n_{\Sigma_{\tau}}^{\mu} .
$$

We thus would obtain

$$
\int_{\Sigma_{\tau}} J_{\mu}^{n_{\Sigma_{\tau}}}\left(\psi_{b}\right) n^{\mu} \leq B \int_{\Sigma_{0}} J_{\mu}^{n_{\Sigma_{0}}}\left(\psi_{b}\right) n^{\mu}
$$

\footnotetext{
${ }^{34}$ Since we are dealing now with general perturbations of Schwarzschild, we shall now use $r$ for what we previously denoted by $r_{\mathrm{Schw}}$. Note that in the special case that our metric is Kerr, this $r$ is different from the Boyer-Lindquist $r$.
} 
Adding (63) and (59), we would obtain

$$
\int_{\Sigma_{\tau}} J_{\mu}^{n_{\Sigma_{\tau}}}(\psi) n^{\mu} \leq B \int_{\Sigma_{0}} J_{\mu}^{n_{\Sigma_{0}}}(\psi) n^{\mu}
$$

provided that we could also estimate say

$$
\int_{\Sigma_{0}} J_{\mu}^{n_{\Sigma_{0}}}\left(\psi_{\sharp}\right) n^{\mu} \leq B \int_{\Sigma_{0}} J_{\mu}^{n_{\Sigma_{0}}}(\psi) n^{\mu} .
$$

4.5.5. Cutoff and decomposition. Unfortunately, things are not so simple!

For one thing, to take the Fourier transform necessary to decompose in frequency, one would need to know a priori that $\psi\left(t^{*}, \cdot\right)$ is in $L^{2}\left(t^{*}\right)$. What we want to prove at this stage is much less. A priori, $\psi$ can in fact grow exponentially in $t^{*}$. In order to apply the above, one must cut off the solution appropriately in a time slab of interest, and then decompose with the Fourier transform and smooth frequency cutoffs, defining $\psi_{b}, \psi_{\sharp}$. These will now satisfy

$$
\square_{g} \psi_{b}=F_{b}, \quad \square_{g} \psi_{\sharp}=F_{\sharp} .
$$

When interpreted for the $\psi_{b}, \psi_{\sharp}$ defined above, the arguments outlined before produce error terms from:

- the inhomogeneous terms $F_{b}, F_{\sharp}$ from (65)

- the fact that we wish to localise estimates (54) and (60) to subregions $\mathcal{H}^{+}\left(\tau^{\prime}, \tau^{\prime \prime}\right)$ and $\mathcal{R}\left(\tau^{\prime}, \tau^{\prime \prime}\right)$ respectively

- the fact that (64) is not exactly true.

To close the continuity argument, it is essential to control these error terms by the non-degenerate energy (the analogue of (30)) one proves to be bounded times a small constant. This is rather technical. For this, use is made of the fact that for metrics in the allowed class sufficiently close to Schwarzschild (in the Kerr case, for $|a| \ll M$ ), one can control a priori the exponential growth rate of $\psi$ to be small. See [62].

4.5.6. Pointwise bounds. Having proven the uniform boundedness of (30), one argues as in the proof of Theorem 4.3 to obtain higher order energy and pointwise bounds. In particular, the positivity property in the computation of Proposition 4.2 is stable. See [62]. This completes the proof of Theorem 4.6.

4.6. Quantitative decay for waves on Kerr. To obtain decay results analogous to Theorem 4.5, one needs to understand trapping. For general perturbations of Schwarzschild of the class considered in Theorem 4.6, it is not a priori clear what stability properties one can infer about the nature of the trapped set, and how these can be exploited. But for the Kerr family itself, the trapping structure can easily be understood, in view of the complete integrability of geodesic flow discovered by Carter [26]. The codimensionality of the trapped set persists, but in contrast to the Schwarzschild case where trapped null geodesics all approach the codimensional-1 
subset $r=3 M$ of spacetime, in Kerr, this codimensionality must be viewed in phase space.

There is a convenient way of doing phase space analysis in Kerr spacetimes, namely, as discovered by Carter [27], the wave equation can be separated. Walker and Penrose [143] later showed that both the complete integrability of geodesic flow and the separability of the wave equation have their fundamental origin in the presence of a Killing tensor. ${ }^{35}$ In fact, as we shall see, in view of its intimate relation with the integrability of geodesic flow, Carter's separation of $\square_{g}$ immediately captures the codimensionality of the trapped set.

The separation of the wave equation requires taking the Fourier transform, and then expanding into oblate spheroidal harmonics. As before, taking the Fourier transform requires cutting off in time. Let us for now argue heuristically. We may decompose

$$
\begin{gathered}
\hat{\psi}_{\diamond<}(\omega, \cdot)=\sum_{m, \ell} R_{m \ell}^{\omega}(r) S_{m \ell}(a \omega, \cos \theta) e^{i m \phi}, \\
\hat{F}(\omega, \cdot)=\sum_{m, \ell} F_{m \ell}^{\omega}(r) S_{m \ell}(a \omega, \cos \theta) e^{i m \phi},
\end{gathered}
$$

where $S_{m \ell}$ are the oblate spheroidal harmonics. For each $m \in \mathbb{Z}$, and fixed $\omega$, these are a basis of eigenfunctions $S_{m \ell}$ satisfying

$$
-\frac{1}{\sin \theta} \frac{d}{d \theta}\left(\sin \theta \frac{d}{d \theta} S_{m \ell}\right)+\frac{m^{2}}{\sin ^{2} \theta} S_{m \ell}-a^{2} \omega^{2} \cos ^{2} \theta S_{m \ell}=\lambda_{m \ell} S_{m \ell},
$$

and, in addition, satisfying the orthogonality conditions with respect to the $\theta$ variable,

$$
\int_{0}^{2 \pi} d \varphi \int_{-1}^{1} d(\cos \theta) e^{i m \phi} S_{m \ell}(a \omega, \cos \theta) e^{-i m^{\prime} \phi} S_{m^{\prime} \ell^{\prime}}(a \omega, \cos \theta)=\delta_{m m^{\prime}} \delta_{\ell \ell^{\prime}}
$$

Here, the $\lambda_{m \ell}(\omega)$ are the eigenvalues associated with the harmonics $S_{m \ell}$. Each of the functions $R_{m \ell}^{\omega}(r)$ is a solution of the following problem

$$
\begin{aligned}
& \Delta \frac{d}{d r}\left(\Delta \frac{R_{m \ell}^{\omega}}{d r}\right)+\left(a^{2} m^{2}+\left(r^{2}+a^{2}\right)^{2} \omega^{2}-\Delta\left(\lambda_{m \ell}+a^{2} \omega^{2}\right)\right) R_{m \ell}^{\omega} \\
& \quad=\left(r^{2}+a^{2}\right) \Delta F_{m \ell}^{\omega} .
\end{aligned}
$$

Note that if $a=0$, we typically label $S_{m \ell}$ by $\ell \geq|m|$ such that

$$
\lambda_{m \ell}(\omega)=\ell(\ell+1) .
$$

With this choice, $S_{m \ell}$ coincides with the standard spherical harmonics $Y_{m \ell}$.

Defining a coordinate $r^{*}$ by

$$
\frac{d r^{*}}{d r}=\frac{r^{2}+a^{2}}{\Delta}
$$

${ }^{35}$ See $[\mathbf{2 9}, \mathbf{9 9}]$ for recent higher-dimensional generalisations of these properties. 
and setting

$$
u(r)=\left(r^{2}+a^{2}\right)^{1 / 2} R_{m \ell}^{\omega}(r), \quad H(r)=\frac{\Delta F_{m \ell}^{\omega}(r)}{\left(r^{2}+a^{2}\right)^{1 / 2}},
$$

then $u$ satisfies

$$
\frac{d^{2}}{\left(d r^{*}\right)^{2}} u+\left(\omega^{2}-V_{m \ell}^{\omega}(r)\right) u=H
$$

where

$$
\begin{aligned}
V_{m \ell}^{\omega}(r)= & \frac{4 M r a m \omega-a^{2} m^{2}+\Delta\left(\lambda_{m \ell}+\omega^{2} a^{2}\right)}{\left(r^{2}+a^{2}\right)^{2}}+\frac{\Delta\left(3 r^{2}-4 M r+a^{2}\right)}{\left(r^{2}+a^{2}\right)^{3}} \\
& -\frac{3 \Delta^{2} r^{2}}{\left(r^{2}+a^{2}\right)^{4}} .
\end{aligned}
$$

Consider the following quantity

$$
Q=f\left(\left|\frac{d u}{d r^{*}}\right|^{2}+\left(\omega^{2}-V\right)|u|^{2}\right)+\frac{d f}{d r^{*}} \operatorname{Re}\left(\frac{d u}{d r^{*}} \bar{u}\right)-\frac{1}{2} \frac{d^{2} f}{d r^{* 2}}|u|^{2} .
$$

Then, with the notation ${ }^{\prime}=\frac{d}{d r^{*}}$,

$$
Q^{\prime}=2 f^{\prime}\left|u^{\prime}\right|^{2}-f V^{\prime}|u|^{2}+\operatorname{Re}\left(2 f \bar{H} u^{\prime}+f^{\prime} \bar{H} u\right)-\frac{1}{2} f^{\prime \prime \prime}|u|^{2} .
$$

The most dangerous frequency range is $|\omega| \geq \omega_{1}, \lambda_{m \ell} \geq \lambda_{1} \omega$ for arbitrary $\omega_{1}>0$ and some $\lambda_{1}>0$.

Proposition 4.6.1. For $|a| \ll M$, then for the above frequency range, $V^{\prime}$ has a unique simple zero $r_{m \ell}^{\omega}$.

This allows to construct for each frequency set $(\omega, m, \ell)$ a function $f$ (vanishing precisely at $r_{m \ell}^{\omega}$ ) such that the resulting $Q^{\prime}$ is nonegative definite. The resulting estimates can be summed to yield an integrated decay estimate precisely as in (39).

Of course, as with the proof of the boundedness theorem, the cutoff in time necessary to implement this argument gives rise to error terms. Having already obtained Theorem 4.6, these error terms can be controlled more easily. See $[\mathbf{6 3}]$.

To turn integrated decay as in (39) into decay of energy and pointwise decay, we must adapt the argument of Section 4.4.2. There are various subtleties. The stability of considerations near the horizon is tricky, since $Z$ is not $C^{1}$. Here, an improved version of Proposition 4.3.1 is essential. It turns out that in view of the ergoregion, one has quadratically growing terms in $t$ in the $K^{Z . w}$. This necessitates a loss of $\delta>0$ in the polynomial decay rate, where however we may take $\delta \rightarrow 0$ as $a \rightarrow 0$. Another subtlety is that to obtain good decay towards null infinity, one must commute with the Schwarzschild angular momentum operators, which are of course no longer Killing. 
We obtain finally $[\mathbf{6 4}, \mathbf{6 3}]$

TheOREM 4.8. Let $(\mathcal{M}, g)$ be Kerr for $|a| \ll M$, D be the closure of its domain of dependence, let $\Sigma_{0}$ be the surface $\mathcal{D} \cap\left\{t^{*}=0\right\}$, let $\psi, \psi^{\prime}$ be initial data on $\Sigma_{0}$ such that $\psi \in H_{\mathrm{loc}}^{s}(\Sigma), \psi^{\prime} \in H_{\mathrm{loc}}^{s-1}(\Sigma)$ for $s \geq 1$, and $\lim _{x \rightarrow i^{0}} \psi=0$, and let $\psi$ be the corresponding unique solution of $\square_{g} \psi=0$. Let $\varphi_{\tau}$ denote the 1-parameter family of diffeomorphisms generated by $T$, let $\tilde{\Sigma}_{0}$ be an arbitrary spacelike hypersurface in $J^{+}\left(\Sigma_{0} \backslash \mathcal{U}\right)$ where $\mathcal{U}$ is an open neighborhood of the asymptotically flat end, ${ }^{36}$ and define $\tilde{\Sigma}_{\tau}=\varphi_{\tau}\left(\tilde{\Sigma}_{0}\right)$. Let $s \geq 3$ and assume

$$
E_{1} \doteq \int_{\Sigma_{0}} r^{2}\left(J_{\mu}^{n_{0}}(\psi)+J_{\mu}^{n_{0}}(T \psi)+J_{\mu}^{n_{0}}(T T \psi)\right) n_{0}^{\mu}<\infty .
$$

Then there exists $a \delta>0$ depending on $a$ (with $\delta \rightarrow 0$ as $a \rightarrow 0$ ) and a $B$ depending only on $\tilde{\Sigma}_{0}$ such that

$$
\int_{\tilde{\Sigma}_{\tau}} J^{N}(\psi) n_{\tilde{\Sigma}_{\tau}}^{\mu} \leq B E_{1} \tau^{-2+2 \delta} .
$$

Now let $s \geq 5$ and assume

$E_{2} \doteq \sum_{|\alpha| \leq 2} \sum_{\Gamma=\left\{T, N, \Omega_{i}\right\}} \int_{\Sigma_{0}} r^{2}\left(J_{\mu}^{n_{0}}\left(\Gamma^{\alpha} \psi\right)+J_{\mu}^{n_{0}}\left(\Gamma^{\alpha} T \psi\right)+J_{\mu}^{n_{0}}\left(\Gamma^{\alpha} T T \psi\right)\right) n_{0}^{\mu}<\infty$ where $\Omega_{i}$ are the angular momentum operators of the Schwarzschild solution with $a=0$. Then

$$
\sup _{\tilde{\Sigma}_{\tau}}|\psi| \leq B \sqrt{E_{2}} \tau^{-1+\delta}, \quad \sup _{\tilde{\Sigma}_{\tau}}|r \psi| \leq B \sqrt{E_{2}} \tau^{(-1+\delta) / 2} .
$$

One can obtain decay for arbitrary derivatives, including transversal derivatives to $\mathcal{H}^{+}$, using additional commutation by $N$. See $[\mathbf{6 4 , 6 3}$ for further details.

4.7. Comments and further reading. Theorem 4.6 was proven in [62]. In particular, this provided the first global result of any kind for general solutions of the Cauchy problem on a (non-Schwarzschild) Kerr background. Theorem 4.8 was first announced at the recent Zürich Clay Summer School on Evolution Equations. Results in the direction of the integrated decay estimate (39) in the Kerr case are independently being studied in work in progress by Tataru-Tohaneanu ${ }^{37}$ and Andersson-Blue.

The best previous results concerning Kerr had been obtained by Finster and collaborators in an important series of papers culminating in [73]. The methods of $[\mathbf{7 3}]$ are spectral theoretic, with many pretty applications of contour integration and o.d.e. techniques. The results of [73] apply not to general solutions of the Cauchy problem, but to individual azimuthal modes, i.e. solutions $\psi_{m}$ of fixed $m$. Under the assumption that $\psi_{m}$ is smooth and

\footnotetext{
${ }^{36}$ This is just the assumption that $\tilde{\Sigma}_{0}$ "terminates" on null infinity

${ }^{37}$ This has since appeared as a preprint [138].
} 
vanishes in a neighborhood of $\mathcal{H}^{+} \cap \mathcal{H}^{-}$, the main result stated in [73] is that

$$
\lim _{t \rightarrow \infty} \psi_{m}(r, t)=0
$$

for any $r>r_{+}$. To obtain a result on the sum over $m$ one can interpolate this with the quantitative boundedness statement Theorem 4.6. It is important to note, however, that the statement of [73] need not restrict to $|a| \ll M$, but concerns the entire subextremal range $|a|<M$. Thus, the statement (67) of $[\mathbf{7 3}]$ is currently the only available global statement concerning azimuthal modes on Kerr spacetimes for large but subextremal $a$.

There has also been interesting work on the Dirac equation $[\mathbf{7 2}, \mathbf{8 0}]$, for which superradiance does not occur, and the Klein-Gordon equation [79]. For the latter, see also Section 5.3.

4.8. The cosmological constant $\Lambda$ and Schwarzschild-de Sitter. Another interesting setting for the study of the stability problem are black holes within cosmological spacetimes. Cosmological spacetimes-as opposed to asymptotically flat spacetimes (See Appendix 2.2.3), which model spacetime in the vicinity of an isolated self-gravitating system-are supposed to model the whole universe. The working hypothesis of classical cosmology is that the universe is approximately homogeneous and isotropic (sometimes known as the Copernican principle [81]). In the Newtonian theory, it was not possible to formulate a cosmological model satisfying this hypothesis. ${ }^{38}$ One of the major successes of general relativity was that the theory allowed for such solutions, thus making cosmology into a mathematical science.

In the early years of mathematical cosmology, it was assumed that the universe should be static. To allow for such static cosmological solutions, Einstein modified his equations (1) by adding a 0'th order term:

$$
R_{\mu \nu}-\frac{1}{2} g_{\mu \nu} R+\Lambda g_{\mu \nu}=8 \pi T_{\mu \nu} .
$$

Here $\Lambda$ is a constant known as the cosmological constant. When coupled with a perfect fluid, this system admits a static, homogeneous, isotropic solution with $\Lambda>0$ and topology $\mathbb{S}^{3} \times \mathbb{R}$. This spacetime is sometimes called the Einstein static universe.

Cosmological solutions with various values of the parameter $\Lambda$ were studied by Friedmann and Lemaitre, under the hypothesis of exact homogeneity and isotropy. Static solutions are in fact always unstable under perturbation of initial data. Typical homogeneous isotropic solutions expand or contract, or both, beginning and or ending in singular configurations. As with the early studies illuminating the extensions of the Schwarzschild metric across the horizon, these were ahead of their time. (See the very recent [115] for a history of this fascinating early period in the history of mathematical

\footnotetext{
${ }^{38}$ It is possible, however, if one geometrically reinterprets the Newtonian theory and allows space to be-say-the torus. See $[\mathbf{1 2 4}]$. These reinterpretations, of course, postdate the formulation of general relativity.
} 
cosmology.) These predictions were taken more seriously with Hubble's observational discovery of the expansion of the universe, and the subsequent evolutionary theories of matter, but the relevance of the solutions near where they are actually singular was taken seriously only after the incompleteness theorems of Penrose and Hawking-Penrose were proven (see Section 2.6).

We shall not go into a general discussion of cosmology here, nor tell the fascinating story of the ups and downs of $\Lambda$-from its adoption by Einstein to his subsequent well-known rejection of it, to its later "triumphant" return in current cosmological models, taking a very small positive value, the "explanation" of which is widely regarded as one of the outstanding puzzles of theoretical physics. Rather, let us pass directly to the object of our study here, one of the simplest examples of an inhomogeneous "cosmological" spacetime, where non-trivial small scale structure occurs in an ambient expanding cosmology. This is the Schwarzschild-de Sitter solution.

4.8.1. The Schwarzschild-de Sitter geometry. Again, this metric was discovered in local coordinates early in the history of general relativity, independently by Kottler [96] and Weyl [145]. Fixing $\Lambda>0,{ }^{39}$ Schwarzschild-de Sitter is a one-parameter family of solutions of the from

$$
-\left(1-2 M / r-\Lambda r^{3}\right) d t^{2}+\left(1-2 M / r-\Lambda r^{3}\right)^{-1} d r^{2}+r^{2} d \sigma_{\mathbb{S}^{2}} .
$$

The black hole case is the case where $0<M<\frac{1}{3 \sqrt{\Lambda}}$. A maximally-extended solution (see $[\mathbf{2 5}, \mathbf{7 7}]$ ) then has as Penrose diagram the infinitely repeating chain:

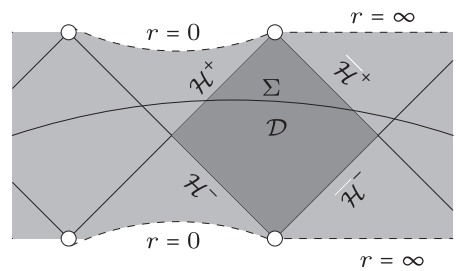

To construct "cosmological solutions" one often takes spatially compact quotients.

4.8.2. Boundedness and decay. The region "analogous" to the region studied previously for Schwarzschild and Kerr is the darker shaded region $\mathcal{D}$ above. The horizon $\overline{\mathcal{H}}^{+}$separates $\mathcal{D}$ from an "expanding" region where the spacetime is similar to the celebrated de-Sitter space. If $\Sigma$ is a Cauchy surface such that $\Sigma \cap \mathcal{H}^{-}=\Sigma \cap \overline{\mathcal{H}}^{-}=\varnothing$, then let us define $\Sigma_{0}=\mathcal{D} \cap \Sigma$, and let us define $\Sigma_{\tau}$ to be the translates of $\Sigma_{0}$ by the flow $\varphi_{t}$ generated by the Killing field $T\left(=\frac{\partial}{\partial t}\right)$. Note that, in contrast to the Schwarzschild or Kerr case, $\Sigma_{0}$ is compact.

\footnotetext{
${ }^{39}$ The expression (69) with $\Lambda<0$ defines Schwarzschild-anti-de Sitter. See Section 5.4.
} 
We have

TheOREM 4.9. The statement of Theorem 4.3 holds for these spacetimes, where $\Sigma, \Sigma_{0}, \Sigma_{\tau}$ are as above, and $\lim _{x \rightarrow i^{0}}|\psi|$ is replaced by $\sup _{x \in \Sigma_{0}}|\psi|$.

Proof. The proof of the above theorem is as in the Schwarzschild case, except that in addition to the analogue of $N$, one must use a vector field $\bar{N}$ which plays the role of $N$ near the "cosmological horizon" $\overline{\mathcal{H}}^{+}$. This is possible in view of the positivity of the surface gravity.

As for decay, we have

THEOREM 4.10. For every $k \geq 0$, there exist constants $C_{k}$ such that the following holds. Let $\psi \in H_{\mathrm{loc}}^{k+1}, \psi^{\prime} \in H_{\mathrm{loc}}^{k}$, and define

$$
E_{k} \doteq \sum_{|(\alpha)| \leq k} \sum_{\Gamma=\left\{\Omega_{i}\right\}} \int_{\Sigma_{0}} J_{\mu}^{n_{\Sigma_{\tau}}}\left(\Gamma^{\alpha} \psi\right) n_{\Sigma_{\tau}}^{\mu} .
$$

Then

$$
\int_{\Sigma_{\tau}} J_{\mu}^{n_{\Sigma_{\tau}}}(\psi) n_{\Sigma_{\tau}}^{\mu} \leq C_{k} E_{k} \tau^{-k}
$$

For $k>1$ we have

$$
\sup _{\Sigma_{\tau}}\left|\psi-\psi_{0}\right| \leq C_{k} \sqrt{E_{k}} \tau^{\frac{-k+1}{2}},
$$

where $\psi_{0}$ denotes the 0 'th spherical harmonic, for which we have for instance the estimate

$$
\sup _{\Sigma_{\tau}}\left|\psi_{0}\right| \leq \sup _{x \in \Sigma_{0}} \psi_{0}+C_{0} \sqrt{E_{0}\left(\psi_{0}, \psi_{0}^{\prime}\right)}
$$

The proof of this theorem uses the vector fields $T, N$ and $\bar{N}$ together with a version of $X$ as multipliers, and requires commutation of the equation with $\Omega_{i}$ to quantify the loss caused by trapping. (Like Schwarzschild, the Schwarzschild-de Sitter metric has a photon sphere which is at $r=3 M$ for all values of $\Lambda$ in the allowed range.) An estimate analogous to (39) is obtained, but without the $\chi$ weight, in view of the compactness of $\Sigma_{0}$. The result of the Theorem follows essentially immediately, in view of Theorem 4.9 and a pigeonhole argument. No use need be made of a vector field of the type $Z$ as in Section 4.4.2. Note that for $\psi=$ constant, $E_{k}=0$, so removing the 0 'th spherical harmonic in (71) is necessary. See [60] for details.

4.8.3. Comments and further reading. Theorem 4.10 was proven in [60]. Independently, the problem of the wave equation on Schwarzschild-de Sitter has been considered in a nice paper of Bony-Häfner [21] using methods of scattering theory. In that setting, the presence of trapping is manifest by the appearance of resonances, that is to say, the poles of the analytic 
continuation of the resolvent. ${ }^{40}$ The relevant estimates on the distribution of these necessary for the analysis of [21] had been obtained earlier by Sá Barreto and Zworski [127].

In contrast to Theorem 4.10, the theorem of Bony-Häfner [21] must assume that $\psi$ is supported away from $\mathcal{H}^{+} \cap \mathcal{H}^{-}$. For these special data, however, the results of $[\mathbf{2 1}]$ obtain better decay than Theorem 4.10 away from the horizon, namely exponential, at the cost of only an $\epsilon$ derivative. The decay results of $[\mathbf{2 1}]$ degenerate at the horizon, in particular, they do not retrieve even boundedness for $\psi$ itself. However, using the result of [21] together with the analogue of the red-shift $Y$ estimate as used in the proof of Theorem 4.10, one can prove exponential decay up to and including the horizon, i.e. exponential decay in the parameter $\tau$. This still requires, however, the restrictive hypothesis of $[\mathbf{2 1}]$ concerning the support of the data. It would be interesting to sort out whether the restrictive hypothesis can be removed from [21], and whether this fast decay is stable to perturbation. There also appears to be interesting work in progress by Sá Barreto, Melrose and Vasy $[\mathbf{1 4 0}]$ on a related problem.

One should expect that the statement of Theorem 4.9 holds for the wave equation on axisymmetric stationary perturbations of Schwarzschild-de Sitter, in particular, slowly rotating Kerr-de Sitter, in analogy to Theorem 4.6.

Finally, we note that in many context, more natural than the wave equation is the conformally covariant wave equation $\square_{g} \psi-\frac{1}{6} R \psi=0$. For Schwarzschild-de Sitter, this is then a special case of Klein-Gordon (74) with $\mu>0$. The analogue of Theorem 4.9 and Theorem 4.10 holds for this equation.

\section{Open problems}

Let us end these notes with a discussion of open problems. Many of these are related to Conjecture 4.1, but all have independent interest.

5.1. The wave equation. The decay rates of Theorem 4.5 are sharp as uniform decay rates in $v$ for any nontrivial class of initial data. On the other hand, it would be nice to obtain more decay in the interior, possibly under a stronger assumption on initial data.

Open Problem 1. Show that there exists a $\delta>0$ such that (31) holds with $\tau$ replaced with $\tau^{-2(1+\delta)}$, for a suitable redefinition of $E_{1}$. Show the same thing for Kerr spacetimes with $|a| \ll M$.

At the very least, it would be nice to obtain this result for the energy restricted to $\tilde{\Sigma}_{\tau} \cap\{r \leq R\}$.

Recall how the algebraic structure of the Kerr solution is used in a fundamental way in the proof of Theorem 4.8. On the other hand, one would

\footnotetext{
${ }^{40}$ In the physics literature, these are known as quasi-normal modes. See [95] for a nice survey, as well as the discussion in Section 4.4.6.
} 
think that the validity of the results should depend only on the robustness of the trapping structure. This suggests the following

Open problem 2. Show the analogue of Theorem 4.8 for the wave equation on metrics close to Schwarzschild with as few as possible geometric assumptions on the metric.

For instance, can Theorem 4.8 be proven under the assumptions of Theorem 4.6? Under even weaker assumptions?

Our results for Kerr require $|a| \ll M$. Of course, this is a "valid" assumption in the context of the nonlinear stability problem, in the sense that if this condition is assumed on the parameters of the initial reference Kerr solution, one expects it holds for the final Kerr solution. Nonetheless, one certainly would like a result for all cases. See the discussion in Section 4.7.

Open problem 3. Show the analogue of Theorem 4.8 for Kerr solutions in the entire subextremal range $0 \leq|a|<M$.

The extremal case $|a|=M$ may be quite different in view of the fact that the surface gravity of the horizon is no longer positive.

Open PROBLEM 4. Understand the behaviour of solutions to the wave equation on extremal Reissner-Nordström, extremal Schwarzschild-de Sitter, and extremal Kerr.

Turning to the case of $\Lambda>0$, we have already remarked that the analogue of Theorems 4.9 and 4.10 should certainly hold in the case of Kerrde Sitter. In the case of both Schwarzschild-de Sitter and Kerr-de Sitter, another interesting problem is to understand the behaviour in the region $\mathcal{C}=J^{+}\left(\overline{\mathcal{H}}_{A}^{+}\right) \cap J^{+}\left(\overline{\mathcal{H}}_{B}^{+}\right)$, where $\mathcal{H}_{A}^{+}, \mathcal{H}_{B}^{+}$are two cosmological horizons meeting at a sphere:

OPEN PROBLEM 5. Understand the behaviour of solutions to the wave equation in region $\mathcal{C}$ of Schwarzschild-de Sitter and Kerr-de Sitter, in particular, their behaviour along $r=\infty$ as $i^{+}$is approached.

Let us add that in the case of cosmological constant, in some contexts it is appropriate to replace $\square_{g}$ with the conformally covariant wave operator $\square_{g}-\frac{1}{6} R$. In view of the fact that $R$ is constant, this is a special case of the Klein-Gordon equation discussed in Section 5.3 below.

5.2. Higher spin. The wave equation is a "poor man's" linearisation of the Einstein equations (3). The role of linearisation in the mathematical theory of nonlinear partial differential equations is of a different nature than that which one might imagine from the formal "perturbation" theory which one still encounters in the physics literature. Rather than linearising the equations, one considers the solution of the nonlinear equation from the point of view of a related linear equation that it itself satisfies.

In the case of the simplest nonlinear equations (say (75) discussed in Section 5.6 below), typically this means freezing the right hand side, i.e. treating 
it as a given inhomogeneous term. In the case of the Einstein equations, the proper analogue of this procedure is much more geometric. Specifically, it amounts to looking at the so called Bianchi equations

$$
\nabla_{[\mu} R_{\nu \lambda] \rho \sigma}=0,
$$

which are already linear as equations for the curvature tensor when $g$ is regarded as fixed. For more on this point of view, see [46]. The above equations for a field $S_{\lambda \mu \nu \rho}$ with the symmetries of the Riemann curvature tensor are in general known as the spin-2 equations. This motivates:

Open problem 6. State and prove the spin-2 version of Theorems 4.6 or 4.8 (or Open problem 1) on Kerr metric backgrounds or more generally, metrics settling down to Kerr.

In addition to [46], a good reference for these problems is [45], where this problem is resolved just for Minkowski space. In contrast to the case of Minkowski space, an additional difficulty in the above problem for the black hole setting arises from the presence of nontrivial stationary solutions provided by the curvature tensor of the solutions themselves. This will have to be accounted for in the statement of any decay theorem. From the "linearisation" point of view, the existence of stationary solutions is of course related to the fact that it is the 2-parameter Kerr family which is expected to be stable, not an individual solution.

5.3. The Klein-Gordon equation. Another important problem is the Klein-Gordon equation

$$
\square_{g} \psi=\mu \psi .
$$

A large body of heuristic studies suggest the existence of a sequence of quasinormal modes (see Section 4.4.6) approaching the real axis from below in the Schwarzschild case. When the metric is peturbed to Kerr, it is thought that essentially this sequence "moves up" and produces exponentially growing solutions. See $[\mathbf{1 4 8 , 6 6}]$. This suggests

Open PROBLEM 7. Construct an exponentially growing solution of (74) on Kerr, for arbitrarily small $\mu>0$ and arbitrary small $a$.

Interestingly, if one fixed $m$, then adapting the proof of Section 4.5, one can show that for $\mu>0$ sufficiently small and $a$ sufficiently small, depending on $m$, the statement of Theorem 4.6 holds for (74) for such Kerr's. This is consistent with the quasinormal mode picture, as one must take $m \rightarrow \infty$ for the modes to approach the real axis in Schwarzschild. This shows how misleading fixed- $m$ results can be when compared to the actual physical problem.

5.4. Asymptotically anti-de Sitter spacetimes. In discussing the cosmological constant we have considered only the case $\Lambda>0$. This is the case of current interest in cosmology. On the other hand, from the 
completely different viewpoint of high energy physics, there has been intense interest in the case $\Lambda<0$. See [76].

The expression (69) for $\Lambda<0$ defines a solution known as Schwarzschildanti-de Sitter. A Penrose diagramme of this solution is given below.

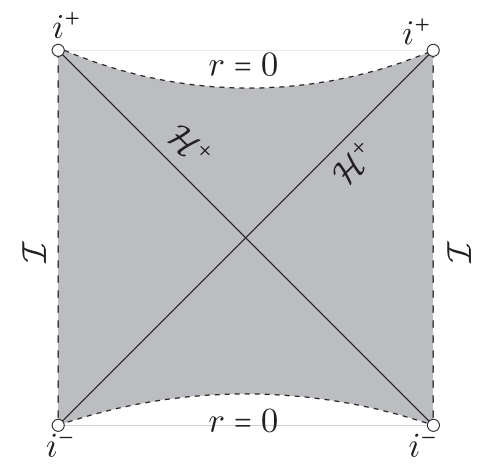

The timelike character of infinity means that this solution is not globally hyperbolic. As with Schwarzschild-de Sitter, Schwarzschild-anti-de Sitter can be viewed as a subfamily of a larger Kerr-anti de Sitter family, with similar properties.

Again, as with Schwarzschild-de Sitter, the role of the wave equation is in some contexts replaced by the conformally covariant wave equation. Note that this corresponds to (74) with a negative $\mu=2 \Lambda / 3<0$.

Even in the case of anti-de Sitter space itself (set $M=0$ in (69)), the question of the existence and uniqueness of dynamics is subtle in view of the timelike character of the ideal boundary $\mathcal{I}$. It turns out that dynamics are unique for (74) only if the $\mu \geq 5 \Lambda / 12$, whereas for the total energy to be nonnegative one must have $\mu \geq 3 \Lambda / 4$. Under our conventions, the conformally covariant wave equation lies between these values. See $[\mathbf{6}, \mathbf{2 3}]$.

Open problem 8. For suitable ranges of $\mu$, understand the boundedness, decay and blow-up properties for solutions of (74) on Schwarzschildanti de Sitter and Kerr-anti de Sitter.

See $[\mathbf{1 0 0 , 2 4}]$ for background. ${ }^{41}$

5.5. Higher dimensions. All the black hole solutions described above have higher dimensional analogues. See $[\mathbf{7 1}, \mathbf{1 1 2}]$. These are currently of great interest from the point of view of high energy physics.

Open problem 9. Study all the problems of Sections 5.1-5.4 in dimension greater than 4 .

Higher dimensions also brings a wealth of explicit black hole solutions such that the topology of spatial sections of $\mathcal{H}^{+}$is no longer spherical. In particular, in 5 spacetime dimensions there exist "black string" solutions,

\footnotetext{
${ }^{41}$ The boundedness problem has since been resolved in the slowly-rotating Kerr-anti de Sitter case. See the very recent paper of Holzegel [85].
} 
and much more interestingly, asymptotically flat "black ring" solutions with horizon topology $S^{1} \times S^{2}$. See [71].

OPEN PROBLEM 10. Investigate the dynamics of the wave equation $\square_{g} \psi=$ 0 and related equations on black ring backgrounds.

5.6. Nonlinear problems. The eventual goal of this subject is to study the global dynamics of the Einstein equations (3) themselves and in particular, to resolve Conjecture 4.1.

It may be interesting, however, to first look at simpler non-linear equations on fixed black hole backgrounds and ask whether decay results of the type proven here are sufficient to show non-linear stability.

The simplest non-linear perturbation of the wave equation is

$$
\square_{g} \psi=V^{\prime}(\psi)
$$

where $V=V(x)$ is a potential function. Aspects of this problem on a Schwarzschild background have been studied by $[\mathbf{1 1 3}, \mathbf{5 8}, \mathbf{1 9}, \mathbf{1 0 6}]$.

OPEN PROBLEM 11. Investigate the problem (75) on Kerr backgrounds.

In particular, in view of the discussion of Section 5.3, one may be able to construct solutions of (75) with $V=\mu \psi^{2}+|\psi|^{p}$, for $\mu>0$ and for arbitrarily large $p$, arising from arbitrarily small, decaying initial data, which blow up in finite time. This would be quite interesting.

A nonlinear problem with a stronger relation to (3) is the wave map problem. Wave maps are maps $\Phi: \mathcal{M} \rightarrow \mathcal{N}$ where $\mathcal{M}$ is Lorentzian and $\mathcal{N}$ is Riemannian, which are critical points of the Lagrangian

$$
\mathcal{L}(\Phi)=\int|d \Phi|_{g_{N}}^{2}
$$

In local coordinates, the equations take the form

$$
\square_{g_{M}} \Phi^{k}=-\Gamma_{i j}^{k} g_{M}^{\alpha \beta}\left(\partial_{\alpha} \Phi^{k} \partial_{\beta} \Phi^{j}\right),
$$

where $\Gamma_{i j}^{k}$ denote the Christoffel symbols of $g_{N}$. See the lecture notes of Struwe [135] for a nice introduction.

Open PRoblem 12. Show global existence in the domain of outer communications for small data solutions of the wave map problem, for arbitrary target manifold $\mathcal{N}$, on Schwarzschild and Kerr backgrounds.

All the above problems concern fixed black hole backgrounds. One of the essential difficulties in proving Conjecture 4.1 is dealing with a black hole background which is not known a priori, and whose geometry must thus be recovered in a bootstrap setting. It would be nice to have more tractable model problems which address this difficulty. One can arrive at such problems by passing to symmetry classes. The closest analogue to Conjecture 4.1 in such a context is perhaps provided by the results of Holzegel [84], which concern the dynamic stability of the 5-dimensional Schwarzschild as a 
solution of (3), restricted under Triaxial Bianchi IX symmetry first studied in $[\mathbf{1 4}]$. In the symmetric setting, one can perhaps attain more insight on the geometric difficulties by attempting a large-data problem. For instance

Open PRoblem 13. Show that the maximal development of asymptotically flat triaxial Bianchi IX vacuum initial data for the 5-dimensional vacuum equations containing a trapped surface settles down to Schwarzschild.

The analogue of the above statement has in fact been proven for the Einstein-scalar field system under spherical symmetry $[\mathbf{3 5}, \mathbf{5 7}]$. In the direction of the above, another interesting set of problems is provided by the Einstein-Maxwell-charged scalar field system under spherical symmetry. For both the charged-scalar field system and the Bianchi IX vacuum system, even more ambitious than Open problem 13 would be to study the strong and weak cosmic censorship conjectures, possibly unifying the analysis of $[\mathbf{4 0 , 5 1 , 5 2}]$. Discussion of these open problems, however, is beyond the scope of the present notes.

\section{Acknowledgements}

Much of the material in these lectures is adapted from a set of lecture notes for a Clay Summer School course in Zürich, written in collaboration with Igor Rodnianski. The author is supported in part by a grant from the European Research Council.

\section{Appendix A. Lorentzian geometry}

The reader who wishes a formal introduction to Lorentzian geometry can consult $[\mathbf{8 1}]$. For the reader familiar with the concepts and notations of Riemannian geometry, the following remarks should suffice for a quick introduction.

A.1. The Lorentzian signature. Lorentzian geometry is defined as in Riemannian geometry, except that the metric $g$ is not assumed positive definite, but of signature $(-,+, \ldots,+)$. That is to say, we assume that at each point $p \in \mathcal{M}^{n+1},{ }^{42}$ we may find a basis $\mathbf{e}_{i}$ of the tangent space $T_{p} \mathcal{M}$, $i=0, \ldots, n$, such that

$$
g=-\mathbf{e}_{0} \otimes \mathbf{e}_{0}+\mathbf{e}_{1} \otimes \mathbf{e}_{1}+\cdots+\mathbf{e}_{n} \otimes \mathbf{e}_{n} .
$$

In Riemannian geometry, the - in the first term on the right hand side would by + .

A non-zero vector $v \in T_{p} \mathcal{M}$ is called timelike, spacelike, or null, according to whether $g(v, v)<0, g(v, v)>0$, or $g(v, v)=0$. Null and timelike vectors collectively are known as causal. There are various conventions for the 0 -vector. Let us not concern ourselves with such issues here.

The appellations timelike, spacelike, null are inherited by vector fields and immersed curves by their tangent vectors, i.e. a vector field $V$ is timelike

\footnotetext{
${ }^{42}$ It is conventional to denote the dimension of the manifold by $n+1$.
} 
if $V(p)$ is timelike, etc., and a curve $\gamma$ is timelike if $\dot{\gamma}$ is timelike, etc. On the other hand, a submanifold $\Sigma \subset \mathcal{M}$ is said to be spacelike if its induced geometry is Riemannian, timelike if its induced geometry is Lorentzian, and null if its induced geometry is degenerate. For a codimension-1 submanifold $\Sigma \subset \mathcal{M}$, at every $p \in M$, there exists a non-zero normal $n^{\mu}$, i.e. a vector in $T_{p} \mathcal{M}$ such that $g(n, v)=0$ for all $v \in T_{p} \Sigma$. It is easily seen that $\Sigma$ is spacelike iff $n$ is timelike, $\Sigma$ is timelike iff $n$ is spacelike, and $\Sigma$ is null iff $n$ is null. Note that in the latter case $n \in T_{p} \Sigma$. The normal of $\Sigma$ is thus tangent to $\Sigma$.

A.2. Time-orientation and causality. A time-orientation on $(\mathcal{M}, g)$ is defined by an equivalence class $[K]$ where $K$ is a continuous timelike vector field, where $K_{1} \sim K_{2}$ if $g\left(K_{1}, K_{2}\right)<0$. A Lorentzian manifold admitting a time-orientation is called time-orientable, and a triple $(\mathcal{M}, g,[K])$ is said to be a time-oriented Lorentzian manifold. Sometimes one reserves the use of the word "spacetime" for such triples. In any case, we shall always consider time-oriented Lorentzian manifolds and often drop explicit mention of the time orientation.

Given this, we may further partition causal vectors as follows. A causal vector $v$ is said to be future-pointing if $g(v, K)<0$, otherwise past-pointing, where $K$ is a representative for the time orientation. As before, these names are inherited by causal curves, i.e. we may now talk of a future-directed timelike curve, etc. Given $p$, we define the causal future $J^{+}(p)$ by

$$
J^{+}(p)=p \cup\{q \in \mathcal{M}: \exists \gamma:[0,1] \rightarrow \mathcal{M}: \dot{\gamma} \text { future-pointing, causal }\}
$$

Similarly, we define $J^{-}(p)$ where future is replaced by past in the above. If $S \subset \mathcal{M}$ is a set, then we define

$$
J^{ \pm}(S)=\cup_{p \in S} J^{ \pm}(p) .
$$

A.3. Covariant derivatives, geodesics, curvature. The standard local notions of Riemannian geometry carry over. In particular, one defines the Christoffel symbols

$$
\Gamma_{\nu \lambda}^{\mu}=\frac{1}{2} g^{\mu \alpha}\left(\partial_{\nu} g_{\alpha \lambda}+\partial_{\lambda} g_{\nu \alpha}-\partial_{\alpha} g_{\nu \lambda}\right)
$$

and geodesics $\gamma(t)=\left(x^{\alpha}(t)\right)$ are defined as solutions to

$$
\ddot{x}^{\mu}+\Gamma_{\nu \lambda}^{\mu} \dot{x}^{\nu} \dot{x}^{\lambda}=0 .
$$

Here $g_{\mu \nu}$ denote the components of $g$ with respect to a local coordinate system $x^{\mu}, g^{\mu \nu}$ denotes the components of the inverse metric, and we are applying the Einstein summation convention where repeated upper and lower indices are summed. The Christoffel symbols allow us to define the covariant derivative on $(k, l)$ tensor fields by

$$
\nabla_{\lambda} A_{\mu_{1} \ldots \mu_{\ell}}^{\nu_{1} \ldots \nu_{k}}=\partial_{\lambda} A_{\mu_{1} \ldots \mu_{\ell}}^{\nu_{1} \ldots \nu_{k}}+\sum_{i=1}^{k} \Gamma_{\lambda \rho}^{\nu_{i}} A_{\mu_{1} \ldots \mu_{\ell}}^{\nu_{1} \ldots \rho \nu_{k}}-\sum_{i=1}^{l} \Gamma_{\lambda \mu_{i}}^{\rho} A_{\mu_{1} \ldots \nu_{\ldots} \ldots \mu_{\ell}}^{\nu_{1} \ldots \nu_{k}}
$$


where it is understood that $\rho$ replaces $\nu_{i}, \mu_{i}$, respectively in the two terms on the right. This defines $(k, l+1)$ tensor. As usual, if we contract this with a vector $v$ at $p$, then we will denote this operator as $\nabla_{v}$ and we note that this can be defined in the case that the tensor field is defined only on a curve tangent to $v$ at $p$. We may thus express the geodesic equation as

$$
\nabla_{\dot{\gamma}} \dot{\gamma}=0 \text {. }
$$

The Riemann curvature tensor is given by

$$
R_{\nu \lambda \rho}^{\mu} \doteq \partial_{\lambda} \Gamma_{\rho \nu}^{\mu}-\partial_{\rho} \Gamma_{\lambda \nu}^{\mu}+\Gamma_{\rho \nu}^{\alpha} \Gamma_{\lambda \alpha}^{\mu}-\Gamma_{\lambda \nu}^{\alpha} \Gamma_{\rho \alpha}^{\mu}
$$

and the Ricci and scalar curvatures by

$$
R_{\mu \nu} \doteq R_{\mu \alpha \nu}^{\alpha}, \quad R \doteq g^{\mu \nu} R_{\mu \nu} .
$$

Using the same letter $R$ to denote all these tensors is conventional in relativity, the number of indices indicating which tensor is being referred to. For this reason we will avoid writing "the tensor $R$ ". The expression $R$ without indices will always denote the scalar curvature. As usual, we shall also use the letter $R$ with indices to denote the various manifestations of these tensors with indices raised and lowered by the inverse metric and metric, e.g.

$$
R_{\mu \nu \lambda \rho}=g_{\mu \alpha} R_{\nu \lambda \rho}^{\alpha}
$$

We say that an immersed curve $\gamma: I \rightarrow \mathcal{M}$ is inextendible if there does not exist an immersed curve $\tilde{\gamma}: J \rightarrow \mathcal{M}$ where $J \supset I$ and $\left.\tilde{\gamma}\right|_{I}=\gamma$.

We say that $(\mathcal{M}, g)$ is geodesically complete if for all inextendible geodesics $\gamma: I \rightarrow \mathcal{M}$, then $I=\mathbb{R}$. Otherwise, we say that it is geodesically incomplete. We can similarly define the notion of spacelike geodesic (in)completeness, timelike geodesic completeness, causal geodesic completeness, etc., by restricting the definition to such geodesics. In the latter two cases, we may further specialise, e.g. to the notion of future causal geodesic completeness, by replacing the condition $I=\mathbb{R}$ with $I \supset(a, \infty)$ for some $a$.

We say that a spacelike hypersurface $\Sigma \subset \mathcal{M}$ is Cauchy if every inextendible causal curve in $\mathcal{M}$ intersects it precisely once. A spacetime $(\mathcal{M}, g)$ admitting such a hypersurface is called globally hyperbolic. This notion was first introduced by Leray [103].

\section{References}

[1] S. Alinhac Energy multipliers for perturbations of Schwarzschild metric Comm. Math. Phys. 288 (2009), no. 1, 199-224.

[2] H. Andreéasson On the Buchdahl inequality for spherically symmetric static shells Comm. Math. Phys. 274 (2007), 399-408.

[3] L. Andersson and V. Moncrief Future complete vacuum spacetimes In: The Einstein equations and the large slace behavior of gravitational fields, 299-330, Birkhäuser, Basel, 2004.

[4] A. Bachelot Gravitational scattering of electromagnetic field by Schwarzschild blackhole Annales de l'I. H. P., section A, 54 (1991), p. 261-320.

[5] A. Bachelot Asymptotic completeness for the Klein-Gordon equation on the Schwarzschild metric, Ann. Inst. H. Poincaré Phys. Théor. 16 (1994), no. 4, 411-441. 
[6] A. Bachelot The Dirac system on the anti-de Sitter universe Commun. Math. Phys. 283 (2008), 127-167.

[7] A. Bachelot and A. Motet-Bachelot Les résonances dun trou noir de Schwarzschild Ann. Inst. H. Poincaré Phys. Théor. 59 (1993), no. 1, 368.

[8] J. M. Bardeen, B. Carter, and S. W. Hawking The four laws of black hole mechanics Commun. Math. Phys. 31 (1973), 161.

[9] R. Bartnik The mass of an asymptotically flat manifold Comm. Pure Appl. Math. 39 (1986), 661-693.

[10] J. D. Bekenstein Generalized second law of thermodynamics in black hole physics, Phys. Rev. D 9 (1974), 3292.

[11] L. Bieri An extension of the stability theorem of the Minkowski space in general relativity, Thesis, ETH, 2007.

[12] H. Buchdahl General relativistic fluid spheres Phys. Rev. 116 (1959), 1027-1034.

[13] P. Bizon, T. Chmaj, A. Rostworowski Late-time tails of a self-gravitating scalar field revisited, in preparation.

[14] P. Bizon, T. Chmaj, B. Schmidt Critical behavior in vacuum gravitational collapse in $4+1$ dimensions Phys. Rev. Lett. 95 (2005), no. 7, 071102, 4 pp.

[15] P. Blue Decay of the Maxwell field on the Schwarzschild manifold, arXiv:0710.4102v1.

[16] P. Blue and A. Soffer Semilinear wave equations on the Schwarzschild manifold. I. Local decay estimates, Adv. Differential Equations 8 (2003), no. 5, 595-614.

[17] P. Blue and A. Soffer Errata for "Global existence and scattering for the nonlinear Schrodinger equation on Schwarzschild manifolds", "Semilinear wave equations on the Schwarzschild manifold I: Local Decay Estimates", and "The wave equation on the Schwarzschild metric II: Local Decay for the spin 2 Regge Wheeler equation", gr-qc/0608073, 6 pages.

[18] P. Blue and A. Soffer Phase space analysis on some black hole manifolds, J. Funct. Anal. 256 (2009), no. 1, 1-90.

[19] P. Blue and J. Sterbenz Uniform decay of local energy and the semi-linear wave equation on Schwarzschild space Comm. Math. Phys. 268 (2006), no. 2, 481-504.

[20] P. Blue and J. Sterbenz A note on the decay properties of linear and non-linear scalar fields on the Schwarzschild/Kruskal space-time, preprint.

[21] J.-F. Bony and D. Häfner Decay and non-decay of the local energy for the wave equation in the de Sitter-Schwarzschild metric, Comm. Math. Phys. 282 (2008), no. 3, 697-719.

[22] H. Bray Proof of the Riemannian Penrose inequality using the positive mass theorem J. Differential Geom. 59 (2001), 177-267.

[23] P. Breitenlohner and D. Z. Freedman Stability in gauged extended supergravity Ann. Phys. 144 (1982), 249-281.

[24] V. Cardoso and O. J. C. Dias Small Kerr-anti-de Sitter black holes are unstable Phys. Rev. D 70, 084011 (2004).

[25] B. Carter Black hole equilibrium states, in Black Holes (Les Houches Lectures), edited B. S. DeWitt and C. DeWitt (Gordon and Breach, New York, 1972).

[26] B. Carter Global structure of the Kerr family of gravitational fields Phys. Rev. 174 (1968), 1559-1571.

[27] B. Carter Hamilton-Jacobi and Schrödinger separable solutions of Einstein's equations Comm. Math. Phys. 10 (1968), 280-310.

[28] E. S. C. Ching, P. T. Leung, W. M. Suen, and K. Young Wave propagation in gravitational systems Phys. Rev. D 52 (1995).

[29] Z. W. Chong, G. W. Gibbons, H. Lu, and C. N. Pope Separability and Killing tensors in Kerr-Taub-NUT-de Sitter metrics in higher dimensions Phys. Lett. B609 124 132 (2005).

[30] Y. Choquet-Bruhat Théoreme d'existence pour certains systèmes d'équations aux dérivées partielles non linéaires Acta Math. 88 (1952), 141-225. 
[31] Y. Choquet-Bruhat, P.T. Chruciel, and J. Loizelet Global solutions of the EinsteinMaxwell equations in higher dimensions Classical and Quantum Gravity 23 (2006), 7383-7394.

[32] Y. Choquet-Bruhat and R. Geroch Global aspects of the Cauchy problem in general relativity Comm. Math. Phys. 14 (1969), 329-335.

[33] D. Christodoulou Reversible and irreversible transformations in black-hole physics Phys. Rev. Lett. 25 (1970), 1596-1597.

[34] D. Christodoulou Violation of cosmic censorship in the gravitational collapse of a dust cloud Comm. Math. Phys. 93 (1984), no. 2, 171-195.

[35] D. Christodoulou A mathematical theory of gravitational collapse Comm. Math. Phys. 109 (1987), 613-647.

[36] D. Christodoulou The formation of black holes and singularities in spherically symmetric gravitational collapse Comm. Pure Appl. Math. 44 (1991), no. 3, 339-373.

[37] D. Christodoulou The nonlinear nature of gravitation and gravitational wave experiments Phys. Rev. Letters 67 (1991) no 12, 1486-1489.

[38] D. Christodoulou Examples of naked singularity formation in the gravitational collapse of a scalar field Ann. of Math. 140 (1994), no. 3, 607-653.

[39] D. Christodoulou Self-gravitating relativistic fluids: a two-phase model Arch. Rat. Mech. Anal. 130 (1995), 343-400.

[40] D. Christodoulou The instability of naked singularities in the gravitational collapse of a scalar field Ann. of Math. 149 (1999), no. 1, 183-217.

[41] D. Christodoulou The action principle and partial differential equations, Ann. Math. Studies No. 146, 1999.

[42] D. Christodoulou On the global initial value problem and the issue of singularities Class. Quantum Gravity 16 (1999) A23-A35.

[43] D. Christodoulou The global initial value problem in general relativity 9th Marcel Grosmann Meeting (Rome 2000), 44-54, World Sci. Publishing, 2002.

[44] D. Christodoulou Mathematical Problems of General Relativity Theory, ETH-Zürich lecture notes, 2003.

[45] D. Christodoulou and S. Klainerman Asymptotic properties of linear field equations in Minkowski space Comm. Pure Appl. Math. 43 (1990), no. 2, 137-199.

[46] D. Christodoulou and S. Klainerman The global nonlinear stability of the Minkowski space Princeton University Press, 1993.

[47] D. Christodoulou The formation of shocks in 3-dimensional fluids EMS Monographs in Mathematics. European Mathematical Society (EMS), Zrich, 2007.

[48] D. Christodoulou The formation of black holes in general relativity arXiv:0805.3880.

[49] P. Chruściel On uniqueness in the large of solutions of Einstein's equations ("strong cosmic censorship") Proceedings of the Center for Mathematical Analysis 27, Australian National University (1991).

[50] P. Chruściel and J. L. Costa On uniqueness of stationary vacuum black holes arXiv:0806.0016.

[51] M. Dafermos Stability and instability of the Cauchy horizon for the spherically symmetric Einstein-Maxwell-scalar field equations Ann. of Math. 158 (2003), no. 3, $875-928$.

[52] M. Dafermos The interior of charged black holes and the problem of uniqueness in general relativity Comm. Pure App. Math. 58 (2005), no. 4, 445-504.

[53] M. Dafermos Spherically symmetric spacetimes with a trapped surface Class. Quantum Grav. 22 (2005), no. 11, 2221-2232.

[54] M. Dafermos Black hole formation from a complete regular past Comm. Math. Phys. 289, 579-596 (2009).

[55] M. Dafermos General Relativity and the Einstein equations. In: Gowers, W. T. (ed.) The Princeton Companion to Mathematics, Princeton University Press, 2008. 
[56] M. Dafermos and G. Holzegel On the nonlinear stability of higher dimensional triaxial Bianchi-IX black holes Adv. Theor. Math. Phys. 10 (2006), no. 4, 503-523.

[57] M. Dafermos and I. Rodnianski A proof of Price's law for the collapse of a selfgravitating scalar field, Invent. Math. 162 (2005), 381-457.

[58] M. Dafermos and I. Rodnianski Small-amplitude nonlinear waves on a black hole background J. Math. Pures Appl. 84 (2005), 1147-1172.

[59] M. Dafermos and I. Rodnianski The redshift effect and radiation decay on black hole spacetimes, Comm. Pure. Appl. Math. 62 (2009), 854-994.

[60] M. Dafermos and I. Rodnianski The wave equation on Schwarzschild-de Sitter spacetimes, arXiv:0709.2766v1 [gr-qc].

[61] M. Dafermos and I. Rodnianski A note on energy currents and decay for the wave equation on a Schwarzschild background, arXiv:0710.0171v1 [math.AP].

[62] M. Dafermos and I. Rodnianski A proof of the uniform boundedness of solutions to the wave equation on slowly rotating Kerr backgrounds, arXiv:0805.4309v1 [gr-qc].

[63] M. Dafermos and I. Rodnianski Lectures on black holes and linear waves, 115 pages, to appear in Proceedings of the Clay Summer School (Zürich June-July 2008), arXiv:0811.0354.

[64] M. Dafermos and I. Rodnianski Polynomial decay for solutions of the wave equation on slowly rotating Kerr backgrounds, in preparation.

[65] T. de Donder La gravifique Einsteinienne Annales de l'Observatoire Royal de Belgique, Brussels, 1921.

[66] S. Detweiler Klein-Gordon equation and rotating black holes Phys. Rev. D 22 (1980), 2323-2326.

[67] J. Dimock Scattering for the Wave equation on the Schwarzschild metric, Gen. Rel. Grav. 17 (1985), 353-369.

[68] J. Dimock and B. Kay Classical and quantum scattering theory for linear scalar field on the Schwarzschild metric I Ann. Phys., 175 (1987), 366-426.

[69] A. Einstein Über Gravitationswellen Königlich Preussiche Akademie der Wissenschaften Berlin Sitzungberichte (1918) 154-167.

[70] A. Einstein On a stationary system with spherical symmetry consisting of many gravitating masses. Ann. Math. 40 (1939), 922-936.

[71] R. Emparan and H. Reall Black Holes in Higher Dimensions Living Rev. Relativity 11, (2008), 6 .

[72] F. Finster, N. Kamran, J. Smoller, S.-T. Yau The long-time dynamics of Dirac particles in the Kerr-Newman black hole geometry Adv. Theor. Math. Phys. 7 (2003), $25-52$.

[73] F. Finster, N. Kamran, J. Smoller, S. T. Yau Decay of solutions of the wave equation in Kerr geometry Comm. Math. Phys. 264 (2006), 465-503.

[74] F. Finster and H. Schmid Spectral estimates and non-selfadjoint perturbations of spheroidal wave operators J. Reine Angew. Math. 601 (2006), 71-107.

[75] H. Friedrich The existence of n-geodesically complete or future complete solutions of Einstein's field equations with smooth asymptotic structure Comm. Math. Phys. 107 (1986), no. 4, 587-609.

[76] G.W. Gibbons Anti-de-Sitter spacetime and its uses In "Mathematical and quantum aspects of relativity and cosmology" (Pythagoreon, 1998), Lecture Notes in Phys. 537, Springer-Verlag, 102142, 2000.

[77] G. W. Gibbons and S. W. Hawking Cosmological event horizons, thermodynamics, and particle creation Phys. Rev. D. 15 (1977), no. 10, 2738-2751.

[78] C. Gundlach, R. Price, and J. Pullin Late-time behavior of stellar collapse and explosions. I. Linearized perturbations, Phys. Rev. D 49 (1994) 883889.

[79] D. Häfner Sur la théorie de la diffusion pour l'équation de Klein-Gordon dans la métrique de Kerr Dissertationes Mathematicae 421 (2003). 
[80] D. Häfner and J.-P. Nicolas Scattering of massless Dirac fields by a Kerr black hole Rev. Math. Phys. 16(1) (2004), 29-123.

[81] S. W. Hawking and G. F. R. Ellis The large scale structure of space-time Cambridge Monographs on Mathematical Physics, No. 1. Cambridge University Press, LondonNew York, 1973.

[82] M. Heusler Black hole uniqueness theorems Cambridge Lecture Notes in Physics, No. 6 Cambridge University Press, Cambridge, 1996.

[83] G. Holzegel The stability problem for black holes Ph. D. Dissertation, University of Cambridge, 2008.

[84] G. Holzegel Stability and decay-rates for the Schwarzschild metric under biaxial perturbations arXiv:0808.3246 [gr-qc].

[85] G. Holzegel On the massive wave equation on slowly rotating Kerr-AdS spacetimes, to appear in Comm. Math. Phys. arXiv:0902.0973.

[86] G. Huisken and T. Ilmanen The inverse mean curvature flow and the Riemannian Penrose inequality J. Differential Geom. 59 (2001), 353-437.

[87] A. Ionescu and S. Klainerman On the uniqueness of smooth, stationary black holes in vacuum arXiv:0711.0040.

[88] P. Karageorgis and J. Stalker Sharp bounds on $2 \mathrm{~m} / \mathrm{r}$ for static spherical objects Class. Quant. Grav. 25 (2008), no. 19, 195021, arXiv:0707.3632v2 [gr-qc].

[89] B. Kay and R. Wald Linear stability of Schwarzschild under perturbations which are nonvanishing on the bifurcation 2-sphere Classical Quantum Gravity 4 (1987), no. 4, 893-898.

[90] R. Kerr Gravitational field of a spinning mass as an example of algebraically special metrics Phys. Rev. Lett. 11 (1963) 237-238.

[91] S. Klainerman Uniform decay estimates and the Lorentz invariance of the classical wave equation Comm. Pure Appl. Math. 38 (1985), 321-332.

[92] S. Klainerman The null condition and global existence to nonlinear wave equations Nonlinear systems of partial differential equations in applied mathematics, Part 1 (Santa Fe, N.M., 1984), 293-326, Lectures in Appl. Math. 23 Amer. Math. Soc., Providence, RI, 1986.

[93] S. Klainerman and I. Rodnianski Rough solutions of the Einstein vacuum equations Ann. of Math. 161 (2005), 1143-1193.

[94] S. Klainerman and I. Rodnianski A Kirchoff-Sobolev parametrix for the wave equation and applications J. Hyperbolic Differ. Equ. 4 (2007), 401-433.

[95] K. Kokkotas and B. Schmidt Quasi-normal modes of stars and black holes Living Rev. Relativity 2 (1999).

[96] F. Kottler Über die physikalischen Grundlagen der Einsteinschen Gravitationstheorie, Ann. Phys. 56 401-462 (1918).

[97] J. Kronthaler Decay rates for spherical scalar waves in the Schwarzschild geometry arXiv:0709.3703.

[98] M. Kruskal Maximal extension of Schwarzschild metric Phys. Rev. 119 (1960), 1743-1745.

[99] D. Kubiznak Hidden symmetries of higher-dimensional rotating black holes Ph.D. Thesis, University of Alberta, September 2008.

[100] H. K. Kunduri, J. Lucietti and H. S. Reall Gravitational perturbations of higher dimensional rotating black holes: tensor perturbations, arXiv:hep-th/0606076v3.

[101] I. Laba and A. Soffer Global existence and scattering for the nonlinear Schrödinger equation on Schwarzschild manifolds Helv. Phys. Acta $\mathbf{7 2}$ (1999), no. 4, 272-294.

[102] G. Lemaitre L'Univers en Expansion Publication du Laboratoire d'Astronomie et de Géodésie de l'Université de Louvain 9 (1932), 171-205.

[103] J. Leray Hyperbolic differential equations The Institute for Advanced Study, Princeton, N. J., 1953. 
[104] H. Lindblad and I. Rodnianski The global stability of Minkowski space-time in harmonic gauge to appear, Ann. of Math.

[105] M. Machedon and J. Stalker Decay of solutions to the wave equation on a spherically symmetric background, preprint.

[106] J. Marzuola, J. Metcalfe, D. Tataru, M. Tohaneanu Strichartz estimates on Schwarzschild black hole backgrounds arXiv:0802.3942v3.

[107] J. Metcalfe Strichartz estimates on Schwarzschild space-times Oberwolfach Reports 44 (2007), 8-11.

[108] H. Minkowski Raum und Zeit Jahresbericht der deutschen MathematikerVereinigung 18 (1909), 75-88.

[109] C. W. Misner, K. S. Thorne, and J. A. Wheeler Gravitation W. H. Freeman and Co., San Francisco, Calif., 1973.

[110] V. Moncrief and D. Eardley The global existence problem and cosmic censorship in general relativity Gen. Rel. Grav. 13 (1981), 887-892.

[111] C. S. Morawetz The limiting amplitude principle Comm. Pure Appl. Math. 15 (1962) 349-361.

[112] R. C. Myers and M. J. Perry Black holes In higher dimensional space-times Ann. Phys. (N.Y.) 172 (1986), 304.

[113] J.-P. Nicolas Non linear Klein-Gordon equation on Schwarzschild-like metrics J. Math. Pures Appl. 74 (1995), 35-58.

[114] J.-P. Nicolas Opérateur de diffusion pou le système de Dirac en métrique de Schwarzschild C. R. Acad. Sci. Paris Sér. I Math. 318 (1994), 729-734.

[115] H. Nussbaumer and L. Bieri Discovering the Expanding Universe Cam. Univ. Press, Cambridge, 2009.

[116] E. Noether Invariante Variationsprobleme Nachr. d. König. Gesellsch. d. Wiss. zu Göttingen, Math-phys. Klasse (1918) 235-257.

[117] J. R. Oppenheimer and H. Snyder On continued gravitational contraction Phys. Rev. 56 (1939), 455-459.

[118] R. Penrose Gravitational collapse and space-time singularities Phys. Rev. Lett. 14, $57-59$.

[119] R. Penrose Gravitational collapse: the role of general relativity Rev. del Nuovo Cimento 1, (1969) 272-276.

[120] R. Penrose Singularities and time asymmetry In "General Relativity-an Einstein Survey" S. Hawking, W. Israel ed., Cambridge University Press, Cambridge, 1979.

[121] F. Planchon and I. Rodnianski, On uniqueness for the Cauchy problem in general relativity, preprint.

[122] R. Price Nonspherical perturbations of relativistic gravitational collapse. I. Scalar and gravitational perturbations Phys. Rev. D (3) 5 (1972), 2419-2438.

[123] T. Regge and J. Wheeler Stability of a Schwarzschild singularity Phys. Rev. 108 (1957), 1063-1069.

[124] A. Rendall Theorems on existence and global dynamics for the Einstein equations Living Rev. Relativity 8 (2005), 6.

[125] A. Rendall Partial differential equations in general relativity Oxford Graduate Texts in Mathematics 16 Oxford University Press, Oxford, 2008.

[126] H. Ringström Future stability of the Einstein-non linear scalar field system Invent. Math. 173 (2008), no. 1, 123-208.

[127] A. Sá Barreto and M. Zworski Distribution of resonances for spherical black holes Math. Res. Lett. 4 (1997), no. 1, 103-121.

[128] J. Schauder Das Anfangswertproblem einer quasilinearen hyperbolischen Differentialgleichung zweiter Ordnung in beliebiger Anzahl yon urtabhängigen Veränderliehen Fundam. Math. 24 (1935).

[129] R. Schoen and S.-T. Yau On the proof of the positive mass conjecture in general relativity Comm. Math. Phys. 16 (1979) 45-76. 
[130] R. Schoen and S.-T. Yau Proof of the positive mass theorem. II. Comm. Math. Phys. 79 (1981), no. 2, 231-260.

[131] K. Schwarzschild Über das Gravitationsfeld eines Massenpunktes nach der Einsteinschen Theorie Sitzungsber. d. Preuss. Akad. d. Wissenschaften 1 (1916), 189-196.

[132] K. Schwarzschild Über das Gravitationsfeld einer Kugel aus inkompressibler Flüssigkeit nach der Einsteinschen Theorie Sitzungsber. d. Preuss. Akad. d. Wissenschaften, Berlin (1916).

[133] K. Stellmacher Zum Anfangswertproblem der Gravitationsgleichungen Math. Ann. 115 (1938), 136-152.

[134] J. Stewart Solutions of the wave equation on a Schwarzschild space-time with localised energy Proc. Roy. Soc. London Ser. A 424 (1989), 239-244.

[135] M. Struwe Wave maps with and without symmetries, Clay lecture notes.

[136] J. L. Synge The gravitational field of a particle Proc. Roy. Irish Acad. 53 (1950), 83-114.

[137] J. L. Synge Relativity: the special theory North-Holland Publishing Co., Amsterdam, 1956.

[138] D. Tataru and M. Tohaneanu Local energy estimate on kerr black hole backgrounds, arXiv:0810.5766.

[139] F. Twainy The Time Decay of Solutions to the Scalar Wave Equation in Schwarzschild Background Thesis. San Diego: University of California 1989.

[140] A. Vasy The wave equation on asymptotically de Sitter-like spaces Oberwolfach Reports 41 (2007), 2388-2392.

[141] R. M. Wald Note on the stability of the Schwarzschild metric J. Math. Phys. 20 (1979), 1056-1058.

[142] R. Wald General relativity University of Chicago Press, Chicago, 1984.

[143] M. Walker and R. Penrose On quadratic first integrals of the geodesic equations for type 22 spacetimes Comm. Math. Phys. 18 (1970), 265-274.

[144] H. Weyl Raum, Zeit, Materie Springer, Berlin, 1919.

[145] H. Weyl Über die statischen kugelsymmetrischen Lösungen von Einsteins kosmologischen Gravitationsgleichungen, Phys. Z. 20 (1919), 31-34.

[146] E. Witten A new proof of the positive energy theorem Comm. Math. Phys. 80 (1981), 381-402.

[147] N. Zipser The global nonlinear stability of the trivial solution of the Einstein-Maxwell equations Ph. D. Thesis, Harvard University, 2000.

[148] T. J. M. Zouros and D. M. Eardley Instabilities of massive scalar perturbations of a rotating black hole Ann. of Phys. 118 (1976), 139. 